\title{
Routes to program thermal expansion in three-dimensional lattice metamaterials built from tetrahedral building blocks
}

\author{
Hang Xu, Amr Farag, and Damiano Pasini ${ }^{(*)}$ \\ Department of Mechanical Engineering, McGill University, 817 Sherbrook St. West, \\ Montreal, Quebec, H3A 0C3, Canada \\ *Email of corresponding author: damiano.pasini@mcgill.ca
}

\begin{abstract}
Thermal expansion can be problematic in manifold applications that require thermal stability, yet it can also be purposely exploited to meet specific directional requirements of thermal deformation. Opportunities to tailor thermal expansion in architected materials exist, but design options that are stiff and provide full directional authority on thermal expansion are currently limited by the structural characteristics of existing concepts. In this work, we report routes to systematically engineer thermally responsive lattice materials that are built from dual-material tetrahedral units that are stiff and strong. Drawing from concepts of vector analysis, crystallography, and tessellation, a scheme is presented for three-dimensional lattices to program desired magnitude and spatial directionality, such as unidirectional, transverse isotropic, or isotropic, of thermal expansion. Demonstrations on thermal expansion and mechanical properties include theoretical, computational, and experimental studies of nine representative concepts, from tetrahedral building blocks to compound unit cells that can tessellate structurally efficient lattices with tunable magnitude and prescribed directionality of thermal expansion.
\end{abstract}

Keywords: thermal expansion, three-dimensional lattices, mechanical metamaterials, structural efficiency.

\section{Introduction}

Systems in extreme thermal environments are susceptible to severe temperature changes. Variations in temperature can lead to undesirable geometric changes in sensitive applications that 
require very fine precision during their working conditions, such as satellite antennas, space telescopes, and large array mirrors (Gilmore, 2002; Yamamoto et al., 2014). As shown in Fig. 1a, systems that operate in space need to be thermally stable and dismiss unexpected thermal deformation, a feature that calls for materials with zero coefficient of thermal expansion (CTE) (Gilmore, 2002; Steeves et al., 2007). In other applications, on the other hand, thermal deformation is highly desired, and here the quest shifts to material systems that can deliberately deform in response to a temperature stimulus dictated by the surrounding environment. This scenario generally translates into a demand for large, positive or negative, CTE (Boatti et al., 2017; Ding et al., 2017; Ge et al., 2016). Examples stemming from a broad and diversely wide range of applications include morphing, e.g. self-shaping, self-folding, and deployable systems (Breger et al., 2015; Stoychev et al., 2011; Zhang et al., 2017), self-assembly (Tibbits, 2012), packaging (Breger et al., 2015; Stoychev et al., 2011), actuation (Mao et al., 2016), structural adaptivity (Ding et al., 2017; Mao et al., 2016; Zhang et al., 2017) and biological devices (Stoychev et al., 2011).

In general, low to zero CTE can be achieved with intrinsically low or negative expansion materials (Miller et al., 2009), used as standalone solid or in a composite form without voids (Sleight, 1998). As shown in Fig. 1a, $\mathrm{ZrW}_{2} \mathrm{O}_{8}$ and related ceramics (Korthuis et al., 1995; Mary et al., 1996) or Invar have low, or even negative, CTE. But most of all have shortcomings that limit their use in applications requiring robustness and durability over a large temperature range. Their faults generally correlate with the material type, and examples include: for ceramics, the brittleness and abrupt failure upon peaks of thermal stresses; for Invar-36 and other metals (e.g. $\mathrm{Fe}_{68} \mathrm{Pd}_{32}$ ) with low CTE, their limited range of temperature (Fig. 1a) (Steeves et al., 2007); and for fiber-reinforced composites, the interface thermal strain and failure caused by the large difference of CTEs between fiber and matrix (Ito et al., 2000). Similarly, for applications 
requiring large CTEs, solid materials can feature intrinsically positive or negative CTEs, such as $\mathrm{Fe}_{72} \mathrm{Pt}_{28}$ shown in Fig. 1a. However, the available range of CTE these materials can offer remain too narrow (about $-10^{1} \mathrm{ppm} /{ }^{\circ} \mathrm{C}$ to $10^{3} \mathrm{ppm} /{ }^{\circ} \mathrm{C}$ (Ashby, 2011)) to induce responsive and large deformations, such as those required for morphing and other applications (Mao et al., 2016; Zhang et al., 2017). An effective alternative to all solids listed above are architected materials made of two solids with distinct CTEs. The rational tuning of their structural geometry and/or selection of their components can generate desired values of CTE from large positive to negative including zero.

Different concepts exist in the literature of CTE tunable architected materials. Each has its own characteristics and performance, and CTE tunability $(\triangle C T E)$ is a metric that can be used to discriminate between the potential of their architecture; $\triangle C T E$ measures the range of CTE attainable via geometric manipulation of a concept for a given pair of constituents (Xu and Pasini, 2016). Whereas no $\triangle C T E$ exists for a single material system, dual material concepts can attain a range of CTE values, and the difference between the upper and lower bound represents $\triangle C T E$. The larger $\triangle C T E$, the broader the prospect of manipulating the material architecture to meet given CTE requirements. In the literature, the majority of concepts are in 2D (Ai and Gao, 2017; Grima et al., 2008; Hopkins et al., 2013; Jefferson et al., 2009; Lehman and Lakes, 2014; Lim, 2005; Miller et al., 2008; Palumbo et al., 2011; Sigmund and Torquato, 1997; Steeves et al., 2007; Vandeperre and Clegg, 2003; Wei et al., 2016; Wu et al., 2016; Xu et al., 2017; Yamamoto et al., 2014) and can accommodate only in-plane thermal deformation, including those resorting to origami (Boatti et al., 2017). Others are three-dimensional (Ai and Gao, 2018; Grima et al., 2008; Lakes, 2007; Lehman and Lakes, 2013; Lim, 2013; Miller et al., 2008; Qu et al., 2017; Steeves et al., 2007; Wang et al., 2016; Wu et al., 2016; Xu and Pasini, 2016) and, compared to the planar concepts, have a range of advantages including the ability to provide low density space filling in 
a protective medium (Wadley, 2006), remarkable heat exchange with larger contact area (Kim et al., 2004), CTE tunability in all spatial directions to yield desired thermal behaviour, such as spatial isotropic expansion (Wu et al., 2016; Xu and Pasini, 2016), and others. The lower part of Fig. 1 also shows the main 3D concepts that can use one building block to construct a series of unit cell topologies with controllable CTE. Fig. $1 \mathrm{~g}$ is a periodic foam built from bi-material strips (Fig. 1b) that are dominated by bending, a characteristic that lowers structural efficiency (i.e. stiffness/mass and strength/mass), and brings about unbounded $\triangle C T E$ (Lakes, 2007; Lehman and Lakes, 2014). Fig. 1h is a stretch-dominated lattice made of nested double-barrelled struts (Fig. 1c) with high structural efficiency but low $\triangle C T E$ due to the relatively modest Poisson effect their struts can attain, i.e. transversal mismatch of thermal expansion brings longitudinal contraction (Lehman and Lakes, 2013). Another concept in Fig. 1i has low specific stiffness (Wang et al., 2016) but can attain high CTE tunability mainly in the negative range (as shown in Fig. 1a for the given pair of constituents $\mathrm{Al}$ and Ti). Concepts built from a bi-material tetrahedron as building block (Fig. 1j), on the other hand, are able to ensure a sizeable CTE tunability, while keeping high structural efficiency due to the axial deformation endured by its members (Miller et al., 2008; Palumbo, 2013; Steeves et al., 2007; Xu and Pasini, 2016). While the concepts listed above are introduced by a number of researchers, others are generated via topology optimization (Sigmund and Torquato, 1997; Watts and Tortorelli, 2017), and feature a fairly complex structure. As shown by some illustrative examples in Fig. 1f and k, their architecture is generally benddominated, thus highlighting a uniaxial specific stiffness that is lower than that of stretch dominated, truss-like concepts.

The majority of the 3D building blocks discussed in the literature (Fig. $1 \mathrm{~b}$ to e), except those developed via topology optimization (Fig. 1f and k) (Watts and Tortorelli, 2017), can tailor the CTE in only one specific direction (horizontal direction for Fig. 1b, axial direction for Fig. 1c, 
and vertical direction for Fig. 1d and e). A more diverse performance with a multitude of tunable CTE directions, such as isotropic CTE, can be obtained by assembling several building blocks into a unit cell (as demonstrated in Fig. $1 \mathrm{~g}$ to $\mathrm{j}$ ). This can be achieved by studying the relationships between building block symmetry, tessellation patterns, and effective thermoelastic properties, a research path yet to be systematically explored in the context of architected materials. While mechanisms for tessellating building blocks into unit cells via reflection and translation are available in the literature (Miller et al., 2008), only special cases have been so far examined, with those inspired by multi-fold rotational symmetry in crystallography able to attain only isotropic CTE (Wei et al., 2016). However, other directional requirements of thermal expansion, such as unidirectional and transverse isotropic, might be also desirable in certain engineering applications.

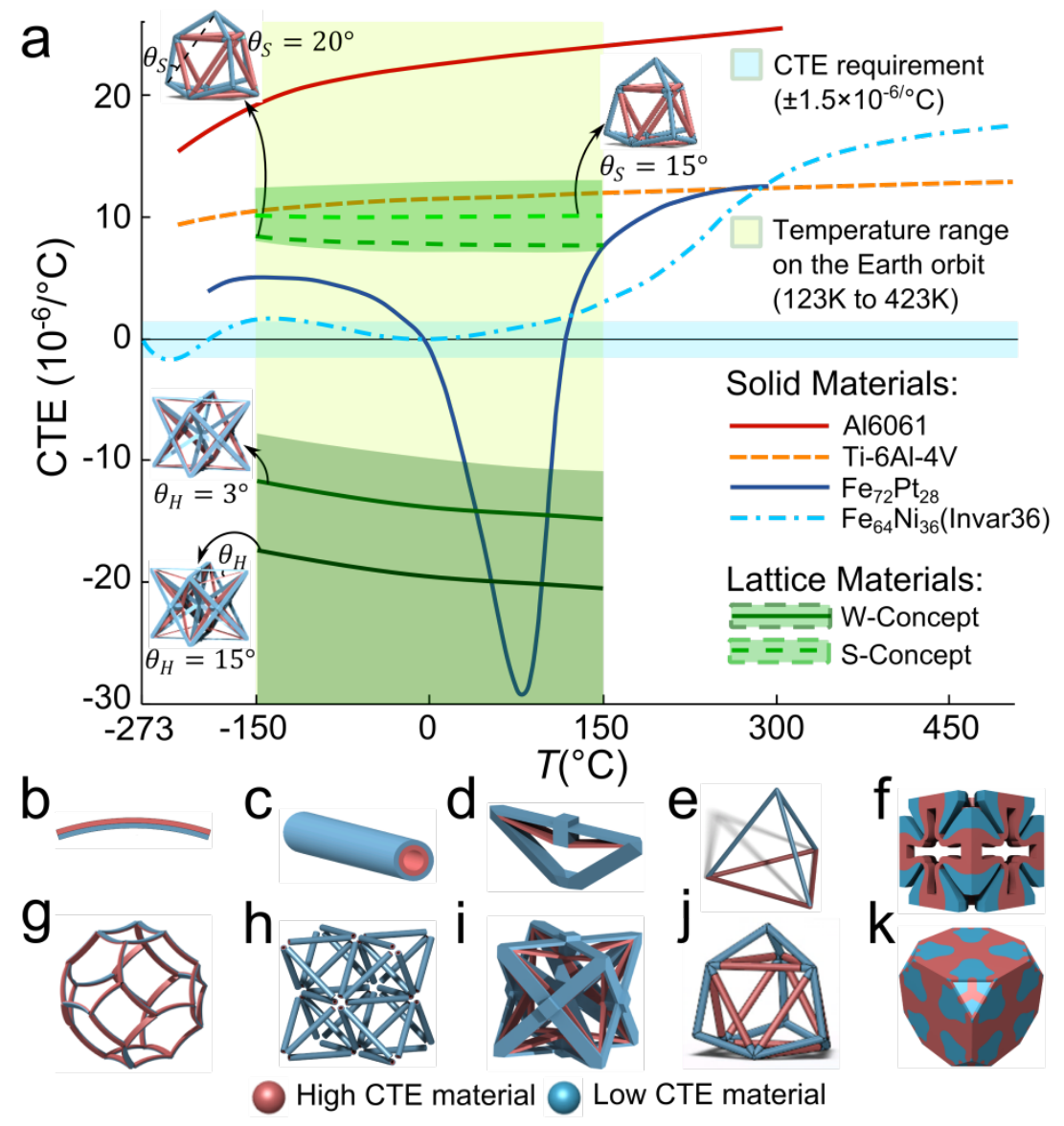

Figure 1: (a) CTE versus temperature for selected solids and architected materials: W-concept (Wang et al., 
2016), S-concept (Steeves et al., 2007), Al6061 (USDOD, 1998), Ti-6Al-4V (USDOD, 1998), and two iron alloys (Liot, 2009). The temperature range on the earth orbit and the CTE requirement for materials used in communication satellites are also shown. Since CTEs of component materials, i.e. Al6061 and Ti-6Al-4V, selected here for demonstrative purposes, change with temperature, the effective CTE of the W-concept and S-concept is also a function of temperature, and its value has been numerically obtained for the temperature range $-150^{\circ} \mathrm{C}$ to $150^{\circ} \mathrm{C}$. (b-k) $\mathrm{CTE}$ tunable building blocks in the literature: (b) a bi-layer strip concept (Lehman and Lakes, 2014), (c) a nested double-barrelled concept (Lehman and Lakes, 2014), (d) a flexure blade concept (Wang et al., 2016), and (e) a tetrahedron concept with a stationary node (Steeves et al., 2007). The corresponding unit cells in 3D are shown in (g) to (j). Concepts constructed via topology optimization: (f) a low-bulk modulus, high thermal expansion design and $(k)$ the maximal modulus design (Watts and Tortorelli, 2017). We also note that a change of the constitutive materials can yield values of the CTE that differ from those in the figure, which are obtained for the given pair of materials: Al6061 and Ti-6Al-4V; prescribing the pair of materials enables a consistent comparison among the relative performance endowed by the architecture of each concept.

The aim of the investigation reported here is twofold: i) to provide a comprehensive understanding of the mechanisms of thermal expansion in spatial lattices built from dual-material tetrahedron building blocks that are not only stiff and strong but also highly CTE tunable, and ii) to offer systematic routes to program their thermal expansion in given directions as required by the application. Concepts of vector analysis are used to express thermal expansion of building blocks and compound units along their principal, or any other, directions. In addition, notions of crystal symmetry are borrowed from crystallography to elucidate the relationship between geometric symmetry and thermal expansion of bi-material lattices assembled from either building blocks or compound units. The proposed framework enables the attainment of three sets of distinct behaviour of directional CTE: i) unidirectional, ii) transverse isotropic, and iii) isotropic. In addition to CTE tunability, closed form expressions are provided for the Young's modulus, shear modulus, buckling and yielding strength of unit cells, here introduced to attain a high level of both CTE tunability and structural efficiency. A total of 21 proof-of-concepts are built and their effective CTE tested via three-dimensional digital image correlation (3D DIC) for model validation; in particular, 12 proof-of-concepts are metallic tetrahedra built from Al6061, Ti-6Al- 
$4 \mathrm{~V}$, and Invar-36, and 9 are polymer unit cell specimens made of PTFE (Polytetrafluoroethylene), acrylic, and ABS (Acrylonitrile Butadiene Styrene). Concluding the paper is a discussion that gauges the trade-off between CTE tunability and specific stiffness, a paramount insight to elucidate for aerospace and other applications.

\subsection{D lattice with low-CTE tetrahedra}

In this section, we draw from concepts of vector analysis to reframe thermal expansion of periodic trusses as a relationship between the material length vector and the thermal displacement vector. These concepts are first applied to the tetrahedron building block with the goal of studying thermal expansion in a three-dimensional space. Then we borrow concepts from crystallography to show how typical relations of symmetry can be used to create unit cells with desired thermal expansion, such as unidirectional, transverse isotropic, or isotropic. These can be further tessellated into spatial trusses or lattices exhibiting prescribed thermoelastic behaviour.

\subsection{Definition of material length vector and thermal displacement vector}

In a solid, a change in temperature can cause the material to change in shape, length, area and volume. Here we show that via the application of the thermal displacement vector, it is possible to draw a simple analogy between the thermal deformation triggered in a solid material and that appearing in a bi-material building block. Below, the basic concepts for a solid material are briefly summarized and then extended to a truss system.

Fig. 2a-I shows the material length vector, $\mathbf{M}$, whose direction defines a referential direction along which the thermal expansion is measured, and whose magnitude describes the initial length, $l_{0}$, i.e. the initial distance between two points, e.g. A and $\mathrm{B}$, in a spatial domain. If point $\mathrm{A}$ is selected as a stationary reference, with a change in temperature $(\Delta T)$, point B can move away from its original location to reach a generic point B' (Fig. 2a-II), creating the vector, $\mathbf{N}$, which is 
defined as the thermal displacement vector. Assuming there is no rigid-body translation or rotation of the material in the domain $\mathrm{A} x_{1} x_{2} x_{3}$, we can represent the thermal strain by the secondorder tensor, $\left[\varepsilon_{i j}\right]$ :

$$
N_{i}=\varepsilon_{i j} M_{j} \quad(i, j=1,2,3)
$$

where the subscript follows the Einstein notation. If $\Delta T$ takes place uniformly throughout the solid (i.e. $\Delta T$ is a scalar), the deformation is uniform, and all the components of $\left[\varepsilon_{i j}\right]$ are proportional to $\Delta T$ such that:

$$
\varepsilon_{i j}=\alpha_{i j} \Delta T
$$

where $\alpha_{i j}$ is the CTE along the direction $i j$. Since $\left[\varepsilon_{i j}\right]$ is a symmetrical second-order tensor, so is $\left[\alpha_{i j}\right]$, which is given in its three-dimensional form by:

$$
\alpha_{i j}=\left[\begin{array}{lll}
\alpha_{11} & \alpha_{12} & \alpha_{13} \\
\alpha_{21} & \alpha_{22} & \alpha_{23} \\
\alpha_{31} & \alpha_{32} & \alpha_{33}
\end{array}\right]
$$

In the most general case where the solid material can freely expand in any direction, $\mathbf{N}$ has components both parallel $\left(N_{\|}\right)$and transverse $\left(N_{\perp}\right)$ to $\mathbf{M}$. The magnitude of the CTE in the direction of $\mathbf{M}$ is given by measuring $N_{\|} /(M \Delta T)$, where $M$ is the length of $\mathbf{M}$ and $N_{\|}=|\mathbf{N}| \cos (\beta)$ (Fig. 2-a) is identical to the length change, $\Delta l$. Then the CTE in the M direction, $\alpha_{\mathbf{M}}$, can be expressed as:

$$
\alpha_{\mathbf{M}}=\frac{\mathbf{M} \cdot \mathbf{N}}{M^{2} \Delta T}
$$

where "." represents the dot product. Eq. (4) shows that for a unit change in temperature the CTE in any arbitrary direction is the thermal extension or contraction, per unit length of a line 
drawn originally in that direction. If the thermal expansion tensor is conveniently expressed with respect to its mutually orthogonal principal axes, then the tensor (Eq. (3)) simplifies to:

$$
\alpha_{i j}=\left[\begin{array}{lll}
\alpha_{1} & & \\
& \alpha_{2} & \\
& & \alpha_{3}
\end{array}\right],
$$

where $\alpha_{1}, \alpha_{2}$, and $\alpha_{3}$ are the principal CTEs in the $x_{1}, x_{2}$, and $x_{3}$ directions. For the remainder of the paper, the material length and the thermal displacement vectors are now abbreviated to ML and TD vectors, respectively, and mathematically expressed as $\mathbf{M}$ and $\mathbf{N}$, respectively. The simple concepts for ML and TD vectors described above for a solid material (Fig. 2a), can be extended to a dual-material triangle truss in 2D (Fig. 2b) and further to a tetrahedron truss in $3 \mathrm{D}$ (Fig. 2c). With a temperature increase, the height rise of the dual-material triangle in Fig. 2b, triggered by the blue elements with low CTE is compensated by the sinking of its top vertex (Point B) due to the higher thermal expansion of the red bar with high CTE. In Fig. 2b-I, the ML vector describes the direction of thermal expansion between the midpoint of the base $\left(\mathrm{O}_{\mathrm{AC}}\right.$, taken here as the reference point) and the apex (point B), which is the only CTE tunable direction in the triangle. By harnessing the CTE values of the solid components, $\alpha_{s 1}$ and $\alpha_{s 2}$, or the skewness of the blue elements, $\theta$, we can tailor the CTE of the dual-material triangle in the vertical direction, so as to assume one of the following value: positive (Fig. 2b-II) with codirectional ML and TD vectors; negative (Fig. 2b-III) with ML and TD vectors in the opposite direction; or zero with a zero TD vector. 
a
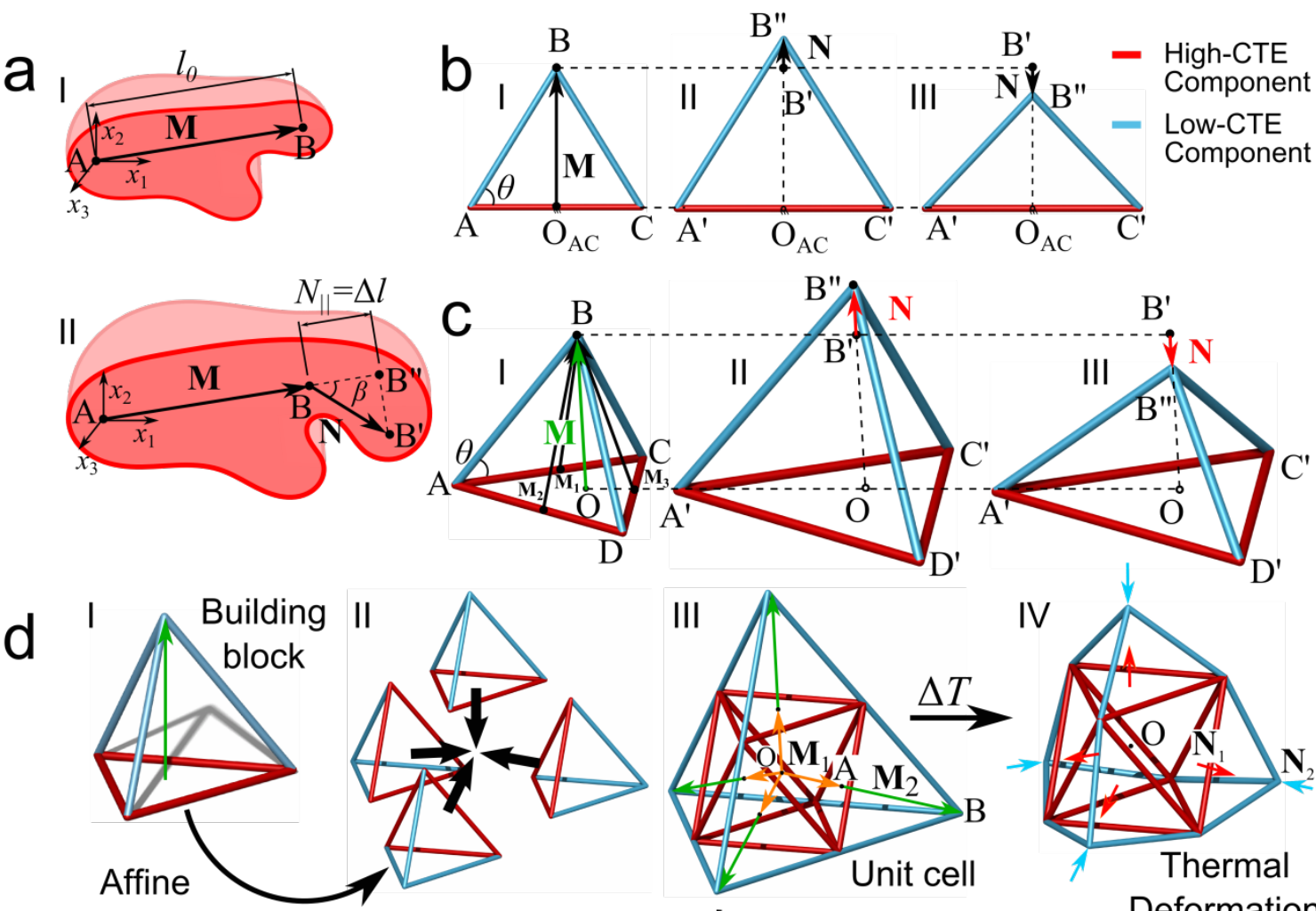

Affine

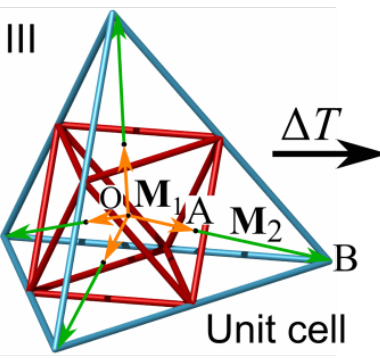

transformations

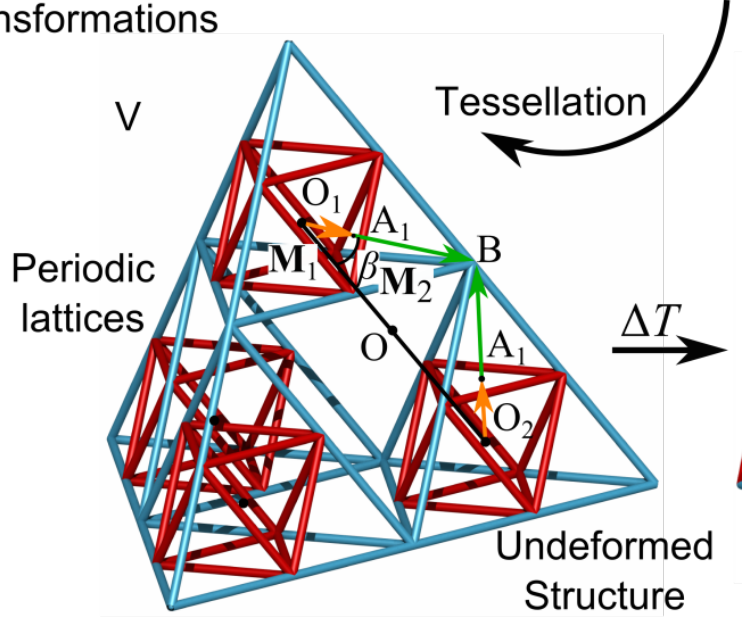

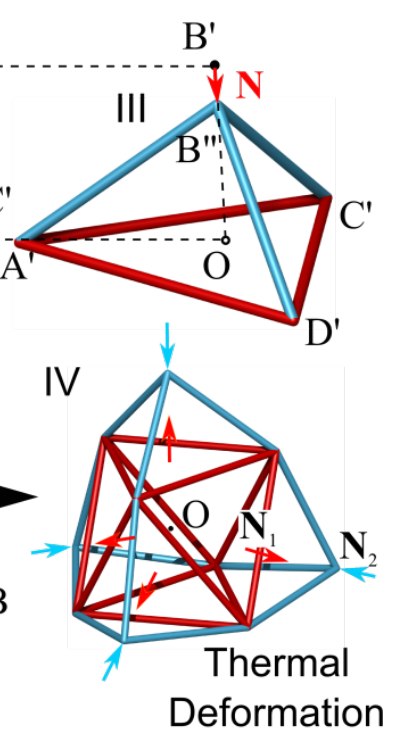

Figure 2: (a) Material length vector, $\mathrm{M}$, and thermal displacement vector, $\mathrm{N}$, for a solid material under a temperature change. Definition of $M$ and $N$ vectors on building blocks in 2D and 3D: (b) 2D low-CTE triangle and (c) 3D low-CTE tetrahedron. (d) $\mathrm{M}$ and $\mathrm{N}$ vectors for an assembled unit cell (d-I to d-IV) and a tessellated system of unit cells (d-V and $d-V I)$ before and after thermal deformation (Steeves et al., 2007). Deformations are visualized considerably larger than the true ones, which are small and within the linear elastic regime.

Similarly in 3D, the $\mathbf{M}$ vector of the dual-material tetrahedron (Fig. 2c-I, (Miller et al., 2008)) is defined by the apex (point $\mathrm{B}$ ) and the centroid of the base triangle (point $\mathrm{O}$, taken as the reference 
point). With a temperature increase, the height rise, i.e. the thermal expansion in the $\mathbf{M}$ direction, triggered by the blue elements is counteracted by the sinking of its apex due to the higher thermal expansion of the red base ( $\triangle \mathrm{ACD}$ ). In this case, all lateral faces (i.e. $\triangle \mathrm{BAC}, \triangle \mathrm{BAD}$, and $\triangle \mathrm{BCD}$ ) of the dual-material tetrahedron are low-CTE triangles (Fig. 2b-I), and the resultant of all three ML vectors of the low-CTE triangles (i.e. $\mathbf{M}_{1}, \mathbf{M}_{2}$, and $\mathbf{M}_{3}$ in Fig. $2 \mathrm{c}-\mathrm{I}$ ) passes through points $\mathrm{O}$ and $\mathrm{B}$. Thus, the direction of the resultant vector given by the sum of $\mathbf{M}_{1}, \mathbf{M}_{2}$, and $\mathbf{M}_{3}$ (each representing the only CTE tunable direction in 2D) is equidirectional to the $\mathbf{M}$ vector of the tetrahedron (green vector in Fig. 2c-I, representing the only CTE tunable direction in 3D). This observation highlights a directional relationship of CTE between a tetrahedron and its triangular faces: the resultant of the 2D ML vectors of the triangles specifies the CTE tunable direction of the tetrahedron, chosen here as the direction of the 3D ML vector. In other words, the direction of the tunable thermal expansion in the triangular faces governs that of the overall tetrahedron. This rule is also useful for locating the ML vector direction of other types of dual-material tetrahedra, along with the CTE tunable directions, as explained in the following section. As per the magnitude, the effective CTE in 3D, similar to the 2D case, can be tailored to be positive (Fig. 2c-II), negative (Fig. 2c-III), and zero through a change in $\theta$ and/or $\alpha_{s 1} / \alpha_{s 2}$. In both 2D and 3D cases, the apex point B can be termed as stationary node (Steeves et al., 2007), since for the zeroCTE case its position relative to the reference point can remain stationary during thermal expansion.

The ML and TD vectors can also express the CTE of an assembly of building blocks, such as the unit cell shown in Fig. 2d-III (Steeves et al., 2007). Here in this exemplifying case, simple affine transformations, in particular translation and rotation (Fig. 2d-II), are used to assemble the dualmaterial building block (Fig. 2d-I) into a unit cell with multiple subunits (Fig. 2d-II). The 
assembly of the four tetrahedra in Fig. 2d-II creates a high CTE octahedron core within the unit cell (Fig. 2d-III). The ML vectors, $\mathbf{M}_{1}$ in orange for the octahedron core, and $\mathbf{M}_{2}$ in green for the tetrahedron (Fig. 2d-III), can be defined between the center of the unit cell, point O, taken as the reference point, and the vertex B, separated by point A (Fig. 2d-III). The overall thermal expansion behavior between $\mathrm{O}$ and $\mathrm{B}$ is given by the sum of the corresponding vectors, $\mathbf{M}_{1}, \mathbf{M}_{2}$, and the TD vectors, $\mathbf{N}_{1}$ and $\mathbf{N}_{2}$, of these two thermally distinct parts, OA and AB. Hence the thermal expansion of the assembled unit cell in Fig. 2d-III can be simply expressed as:

$$
\alpha_{\mathbf{M}}=\frac{\sum_{k}\left(\mathbf{M}_{k} \cdot \mathbf{N}_{k} / M_{k}\right)}{\Delta T \sum_{k} M_{k}} \quad(k=1,2) \text {. }
$$

We now show that the above ML and TD vectors can also be applied to desired 3D tessellations of compound units. Fig. $2 \mathrm{~d}-\mathrm{V}$ shows an example with a periodic truss assembled with the compound cell of Fig. 2d-III. The distance between the centers of any adjacent unit cells, such as between centers $\mathrm{O}_{1}$ and $\mathrm{O}_{2}$, can be accessed via $\mathrm{ML}$ and TD vectors, as shown in Fig. 2d-V and VI. In this case, the thermal expansion of the spatial truss can be simply expressed as:

$$
\alpha_{\mathrm{O}_{1} \mathrm{O}_{2}}=\frac{\sum_{k} N_{\| k}}{\Delta T \sum_{k} M_{\| k}} \quad(k=1,2),
$$

where $N_{\| k}$ and $M_{\| k}$ are the components of $\mathbf{N}_{k}$ and $\mathbf{M}_{k}$ parallel to $\mathrm{O}_{1} \mathrm{O}_{2}$, respectively. We remark here that since the projection cosine of both $N_{\| k}$ and $M_{\| k}$, i.e. $\cos \beta$, is identical, the terms containing the directional angle, $\beta$ in Fig. $2 \mathrm{~d}-\mathrm{V}$ and VI, cancel out in both the numerator and denominator of Eq. (7). No matter which direction is considered, the CTE for this cell topology is identical in all directions, thus equaling the effective CTE evaluated by Eq. (6), i.e. $\alpha_{\mathrm{O}_{1} \mathrm{O}_{2}}=\alpha_{\mathbf{M}}$. We can conclude that since the thermal expansions between centers of any adjacent 
unit cells are identical, the overall CTE of the truss material shown in Fig. 2d-V is thermally isotropic and the magnitude can be evaluated by the vector analysis explained above.

The concept presented in Fig. 2c and d are given as examples to show the handiness of using vectors to visualize and analyze thermal expansion of a periodic truss built from single units as well as a more complex assembly of compound units. In both cases, the primitive building block is a tetrahedron that features a distinct arrangement of solids in its bars. But this is just one among many other material layouts that are feasible. The following sections examine all the possible material arrangements that can appear in the strut of a dual-material tetrahedron, each defining a building block with its own specific CTE profile.

\subsection{Exploration of dual-material tetrahedra}

The simplest $3 \mathrm{D}$ hinged structure that is free to deform upon temperature changes is a tetrahedron. Six types of rods can make up its frame, and different combinations of component materials or dimensions are possible. Below we examine all the material permutations that can occur in the struts of a dual-material tetrahedron, and study the relation that each of these has with the CTE along its principal axes. The goal here is to provide a foundational basis to understand other, more complicated, truss-like materials.

\subsubsection{Thermal deformation mode of dual-material tetrahedra}

Let us consider a tetrahedron made of six rods, each made of a material with either low or high CTE, but both positive. Fig. 3 shows all the possible material permutations that can appear by simply switching the position of red rods with high-CTE, $\alpha_{s 1}$, and blue rods with low-CTE, $\alpha_{s 2}$.

In total, there are eleven possible arrangements, each visualized by a tetrahedron from Fig. 3a to k. For monomaterial tetrahedra (e.g. Fig. 3a and k), the thermal expansion is uniform; as there is no thermal mismatch, no opportunity exists to tailor the CTE. For the remaining tetrahedra, on 
the other hand, CTE tunability is possible as the effective CTE can be adjusted in one or more directions. To ease the CTE assessment, we propose to classify the nine tetrahedra into three groups:

I. Low-CTE tetrahedra (Fig. 3b to e), which can yield an effective CTE that is lower than the CTEs of both the component materials;

II. Intermediate-CTE tetrahedron (Fig. 3f), the only one able to attain the effective CTE of a value between the CTEs of the two components only;

III. High-CTE tetrahedra (Fig. 3g to j), with effective CTEs higher than both the CTEs of the components.

For the sake of simplicity, the main body of the paper examines only the low-CTE cases (Fig. 3b to e), as the analysis of high-CTE cases can be easily completed by switching the material properties. The analysis of the second group (Fig. 3f) is given in Appendix A.

The $\mathbf{M}$ and $\mathbf{N}$ vectors introduced in the previous section now become handy in evaluating the thermal expansion of all tetrahedra in Fig. 3. The direction of the $3 \mathrm{D} \mathbf{M}$ vector with $\mathrm{CTE}$ tunability is controlled by the resultant of the 2D ML vectors of its triangular faces, and can be purposely chosen to align along one of the principal axes of a tetrahedron. As explained in later sections, this choice eases the assembly of building blocks in spatial lattices and helps identify the direction of CTE tunability.

Fig. 3b-I shows a tetrahedron in which one of the rods possesses high-CTE as opposed to the rest with low-CTE. Two low-CTE triangles equivalent to those in Fig. 2 b can be found in Fig. 3b-I, $\Delta$ $\mathrm{ACD}$ (purple face) and $\triangle \mathrm{BCD}$ (yellow face), where only the undeformed configuration is shown to emphasize their ML vector, $\mathbf{M}_{1}$ and $\mathbf{M}_{2}$ (marked as black vectors), whose resultant sets the direction of the $\mathbf{M}$ (green arrow) and $\mathbf{N}$ vector (red arrow) for this tetrahedron. The $\mathrm{M}$ vector visualized in Fig. 3b-I is aligned with the direction of CTE tunability, i.e. the local coordinate 
axis $z$, and show the thermal expansion direction of the deformed tetrahedron (Fig. 3bII). For the zero-CTE case, points A and B are stationary nodes with respect to the line CD in the low-CTE triangles. Analogously, the relative position, including the minimum distance and skew angle between the two skewed lines, $\mathrm{AB}$ and $\mathrm{CD}$, can be constant under a temperature change. We can thus name the pair of lines $\mathrm{AB}$ and $\mathrm{CD}$ as stationary lines (SL), and call the tetrahedron shown in Fig. $3 \mathrm{~b}$ as a tetrahedron with stationary lines (TL-1). Under the pin-jointed assumption, through Eq. (4) we can obtain its effective CTEs as:

$$
\left\{\begin{array}{c}
\alpha_{x}=\alpha_{s 1} \\
\alpha_{y}=\alpha_{s 2} \\
\alpha_{z, T L-1}=\frac{\alpha_{s 2}-\left(\alpha_{s 1}+\alpha_{s 2}\right) \cos ^{2} \theta_{T L-1}}{1-2 \cos ^{2} \theta_{T L-1}}
\end{array}, \theta_{T L-1} \in\left(45^{\circ}, 90^{\circ}\right) .\right.
$$

Fig. 3c and d show two other possible ways by which we can permute the positions of high- and low-CTE bars. Here two high-CTE bars and four low-CTE bars make up the unit: the high-CTE bars of the former meet at one of their vertices, while the high-CTE bars of the latter are not in contact. Let us first examine Fig. 3c, where the tetrahedron has two low-CTE triangles: $\triangle \mathrm{ABC}$ and $\triangle \mathrm{ACD}$. We consider $\mathbf{O}^{\prime} \mathbf{A}$, the resultant of the two corresponding 2D ML vectors ( $\mathbf{M}_{1}$ and $\mathbf{M}_{2}$ ), as the direction of $\mathbf{M}$, and $\mathrm{O}$ as the fixed reference point. After thermal expansion, the deformation mode in Fig. 3c shows the tetrahedron is no longer a regular triangular pyramid. The $\mathbf{N}$ vector, i.e. the change of the relative location between point $\mathrm{A}$ and the base $\triangle \mathrm{BCD}$, indicates the CTE in the vertical direction can be tuned, only while being subjected to shear deformation due to the thermally asymmetric nature of this tetrahedron. For this reason, its thermoelastic properties are given in Appendix A.

We now turn to the tetrahedron in Fig. 3d, where all four triangles have low CTE. Here, the resultant vector of their four 2D ML vectors passes through the midpoints of the two high-CTE 
bars. Similar to the case shown in Fig. 3b, for the zero-CTE case, the relative location, including the minimum distance and the skew angle between lines $\mathrm{AB}$ and $\mathrm{CD}$, can be kept stationary during thermal expansion (Fig. 4b). Thus, the tetrahedron in Fig. 3d has a pair of SLs that can be used for CTE tailoring. The effective CTEs of this tetrahedron with stationary lines (TL-2) can be obtained by applying Eq. (4) under the pin-jointed assumption as:

$$
\left\{\begin{array}{c}
\alpha_{x}=\alpha_{y}=\alpha_{s 1} \\
\alpha_{z, T L-2}=\frac{\alpha_{s 2}-2 \alpha_{s 1} \cos ^{2} \theta_{T L-2}}{1-2 \cos ^{2} \theta_{T L-2}}, \theta_{T L-2} \in\left(45^{\circ}, 90^{\circ}\right) .
\end{array}\right.
$$

For a tetrahedron with three high-CTE bars and three low-CTE bars, there are three possible ways by which bars can be arranged (Fig. 3e, f, and g). In the arrangement shown in Fig. 3e, the high-CTE bars are connected in a loop. During thermal expansion, the relative location between point $\mathrm{A}$ and the base $\triangle \mathrm{BCD}$ can be kept stationary, as discussed in the previous section. Thus, point $\mathrm{A}$ is a stationary node (SN), and Fig. 3e shows a tetrahedron with a stationary node (TN). The effective CTEs of TN under the pin-jointed assumption can be expressed as:

$$
\left\{\begin{array}{c}
\alpha_{x}=\alpha_{y}=\alpha_{s 1} \\
\alpha_{z, T N}=\frac{3 \alpha_{s 2}-4 \alpha_{s 1} \cos ^{2} \theta_{T N}}{3-4 \cos ^{2} \theta_{T N}}, \theta_{T N} \in\left(30^{\circ}, 90^{\circ}\right) .
\end{array}\right.
$$

The case where the high-CTE bars and low-CTE bars are in series, as in Fig. $3 f$ (group two: intermediate tetrahedron), is also given in Appendix A. Fig. $3 \mathrm{~g}$ shows the opposite configuration of Fig. 3e, and hence this building block is used to obtain high-CTE. Similarly, Fig. 3j, h, and i are the counterparts of Fig. $3 \mathrm{~b}$, c, and $\mathrm{d}$, and thus these tetrahedra are viable for high-CTE systems. The close-form equations for the CTE evaluation of high-CTE group can be obtained via switching $\alpha_{s 1}$ and $\alpha_{s 2}$ in the equations of the low-CTE group. 


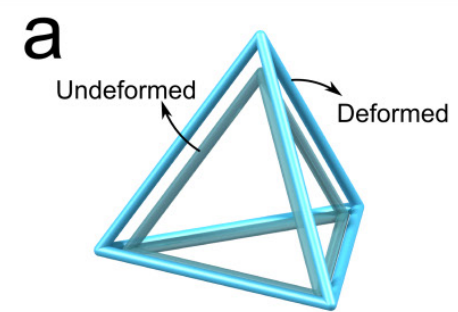

Low CTE material

High CTE material
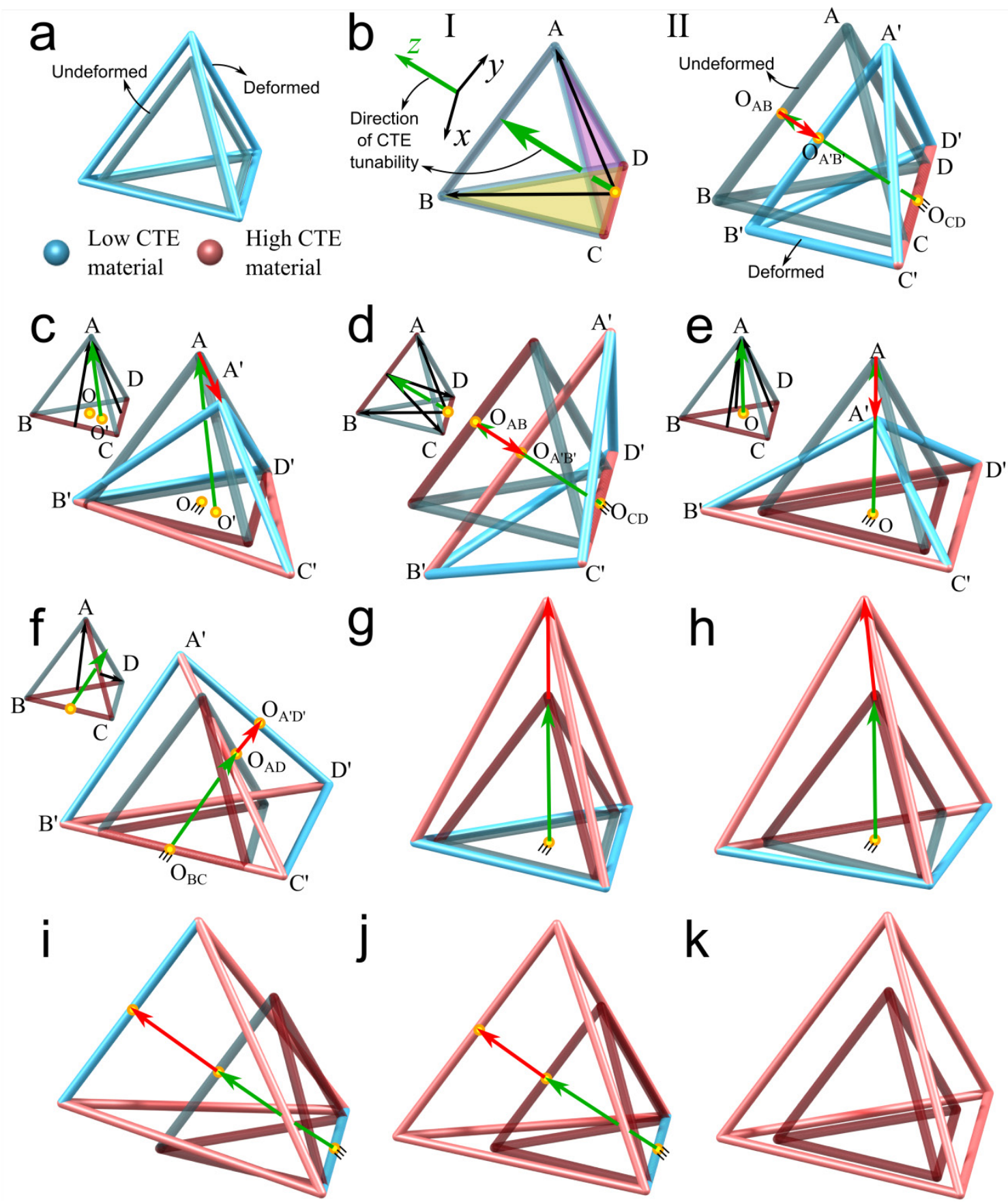

Figure 3: Exploration of dual-material tetrahedra made of permutations of low (blue) and high (red) CTE bars. (a): monomaterial low-CTE tetrahedra with no CTE tunability; (b) to (e): tetrahedra with low-CTE; (f): a tetrahedron with intermediate CTE; and (g) to (j): tetrahedra with high-CTE; (k): monomaterial high-CTE tetrahedra with no CTE tunability. Both undeformed and deformed structures are depicted in each subfigure from (a) to (k). Black arrows represent 2D ML vectors. Green arrows represent 3D $M$ vectors along the direction of CTE tunability, and red arrows $\mathrm{N}$. 


\subsubsection{CTE of low-CTE tetrahedron}

The CTE of the regular tetrahedra presented above can be adjusted by manipulating both material and geometric variables, in given directions. Evidently, the pin-jointed assumption used to derive Eqs. (8) to (10) reduces the CTE-dependent variables to only the skew angle and the CTE of the components. This section examines the role of these two CTE-dependent variables on the effective CTE of building blocks for both high- and low-CTE concepts (considering Fig. 3g to j are the counterparts of $\mathrm{b}$ to e). We examine three CTE-tunable building blocks with different linear-dominant thermal deformation modes along the principal direction: TL-1 (Fig. 3b), TL-2 (Fig. 3d), and TN (Fig. 3e). The zero-CTE cases of these building blocks are also visualized in Fig. 4a, b, and c before and after thermal expansion; each tetrahedron is orientated to keep the $\mathbf{M}$ (green) vector along the vertical direction, demonstrating the concepts of stationary line and stationary node. 
TL-1 Building Block

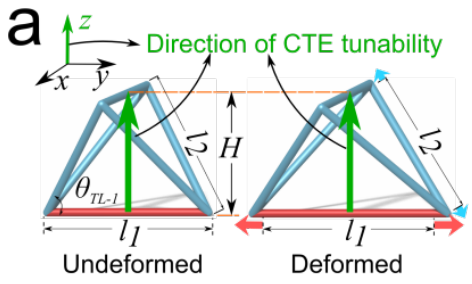

d CTE in the direction of CTE tunability

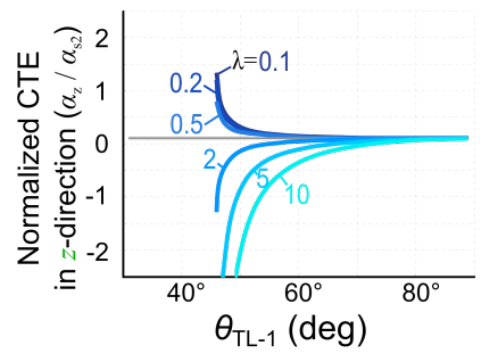

g CTE domain along $x, y, z$

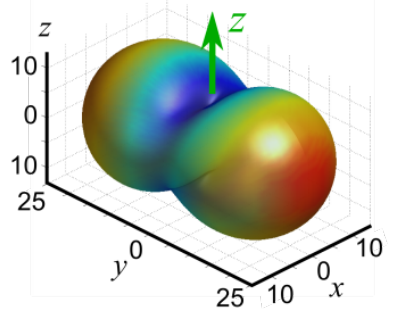

TL-2 Building Block

b

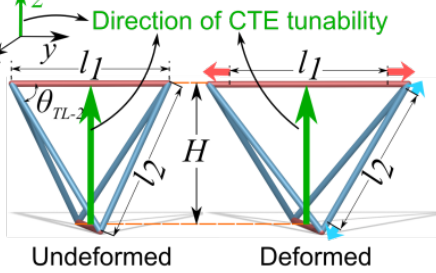

e CTE in the direction of CTE tunability

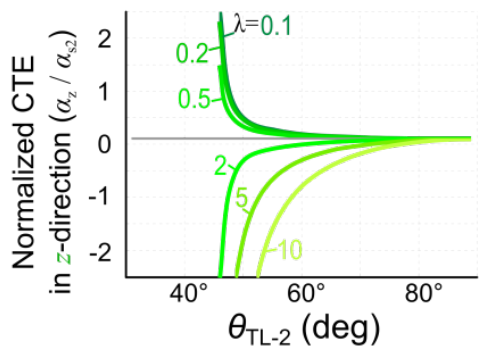

h CTE domain along $x, y, z$

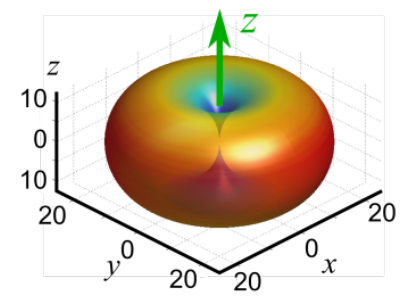

TN Building Block

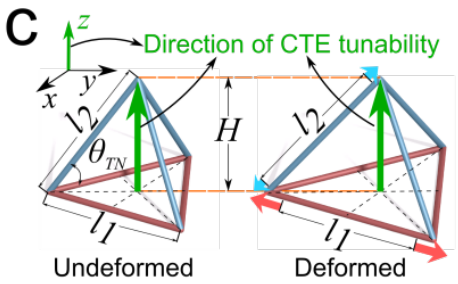

f CTE in the direction of CTE tunability

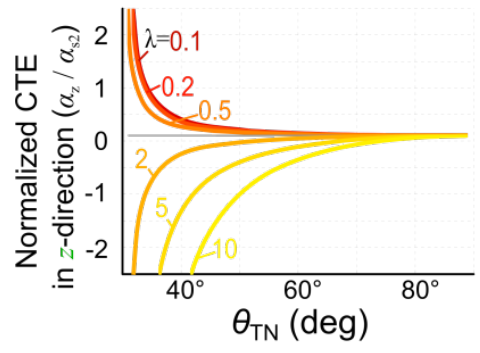

I CTE domain along $x, y, z$

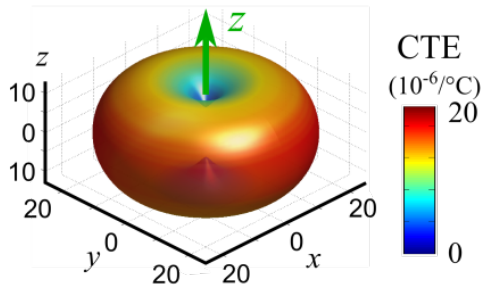

- High CTE material - Low CTE material

Figure 4: Tetrahedra with zero CTE in the principal direction (z-direction): (a) TL-1, (b) TL-2, and (c) TN with undeformed frame in (I) and thermal deformations with zero CTE on the z-direction in (II). The normalized CTEs of Low-CTE tetrahedra along the vertical direction vary with respect to the skew angle and ratio of the component CTE ( $\lambda=\alpha_{s 1} / \alpha_{s 2}$ ): (d) TL-1, (e) TL-2, and (f) TN. CTE magnitude plotted in polar coordinate system with respect to the principal directions, i.e. $x, y$, and $z$; the semi-axes of each ellipsoid are the CTE coefficients, $\left|\alpha_{x}\right|,\left|\alpha_{y}\right|$, and $\left|\alpha_{z}\right|$ of the corresponding tetrahedra with $\lambda=2$ (A high-CTE material of $\alpha_{s 1}=20 \times 10^{-6} /{ }^{\circ} \mathrm{C}$ and a low-CTE material of $\alpha_{s 2}=10 \times 10^{-6} /{ }^{\circ} \mathrm{C}$ are taken here as representative components): (g) TL-1, (h) TL-2, and (i) TN.

Fig. 4d, e, and f, show the effective CTEs of both low- and high-CTE tetrahedra along the $z$ direction (shown in Fig. 4a to c) against the skew angles. From previous section, we recall that the $\mathbf{M}$ vector direction (green arrow), i.e. $z$-direction, is the only CTE tunable principle direction while along the others the CTE is that of the constituent solids. As depicted in Fig. 4d, e, and f, 
$\alpha_{z}$ of the building blocks can be tuned by changing the skew angles to cover a large range of values, from large negative/positive to approximately zero, demonstrating a sizeable CTE tunability. Fig. $4 \mathrm{~d}$ to $\mathrm{f}$ also demonstrate that the CTE in the $z$-direction depends on the CTE ratio of the constituent materials, $\lambda=\alpha_{s 1} / \alpha_{s 2}$. If the skew angle, $\theta$, is given, the larger/smaller the $\lambda$, the lower (for low CTE concept, Fig. 3b, d, and e) or higher (for high CTE concept, Fig. 3g, i, and $\mathrm{j}$ ) the effective CTE, respectively. Hence the greater the CTE distinction of the constituent solids, the higher the CTE tunability. The magnitude of the three ellipsoids shown in Fig. 4g, h, and $\mathrm{i}$ illustrates that the direction of the tetrahedron $\mathbf{M}$ vector (z-direction), i.e. the direction of CTE tunability, follows the direction of a principal axis, i.e. the direction that can yield the lowest CTE magnitude for the case of $\lambda=2$ (whereas the highest CTE magnitude will be for $\lambda<1$ ). The aforementioned building blocks distinguished by their own tunable characteristics lay the foundation for designing stiff and strong spatial lattices with tunable CTE, as shown in the following section.

\subsection{Generation of 3D bi-material lattices with desired thermal expansion}

Here we propose a scheme that systematically uses the building blocks, TL-1, TL-2, or TN, along with their $\mathbf{M}$ and $\mathbf{N}$ vectors in the CTE tunable direction to program thermal expansion in spatial lattices with high specific stiffness and strength. Notions of crystal symmetry, in particular from the crystallographic point groups, are applied to the building blocks and used to assemble more complex truss systems that can meet desired CTE requirements. To illustrate the scheme, subsections 2.3.1 to 2.3.3 present nine examples of lattices that are engineered to attain unidirectional, transverse isotropic, or isotropic controllable CTE. Before doing so, though, we first elucidate the relation between the geometric symmetry in a unit cell and the directions of its thermal expansion, which are typically set a priori by a given application. 
In a general case, the thermal expansion requirements a system should attain can be specified by: (i) the magnitude and sign of its CTE, which can be large positive, near zero, or negative, and/or (ii) the thermal expansion directionality, defining its anisotropic or isotropic behaviour. The former is a requirement mainly governed by the geometry and components of the building blocks, as elucidated in sections 2.1 and 2.2. The latter correlates with the directions of the CTE properties that are governed by the assembly rules of tetrahedral building blocks, such as orientation, symmetry operations, and relative position, as explained below.

There is a direct relation between the geometric symmetry of building blocks in the unit cell and the symmetry of elements of its thermal expansion tensor. To understand this, notions of crystallographic point groups become handy here. As the crystal properties are governed by the crystal symmetry, analogously the CTE properties of a lattice can be inferred by the geometric relationships of symmetry between building blocks. Following the Neumann's Principle: if a crystal is invariant with respect to certain symmetry operations, its physical properties must also be invariant with respect to the same symmetry operations. This is shown in Tab. 1 for several systems, where the effect of crystal symmetry on the second-rank tensor properties is described by the reduction of independent components in its symmetrical tensor. For example, for a crystal with cubic symmetry that is invariant with respect to four 3-fold axes, the CTE can be expressed by a second-rank tensor, $\boldsymbol{\alpha}$, where the number of independent coefficients decreases from six to one; hence the CTE is isotropic. Another example is for a crystal with trigonal symmetry (Fig. 5a), where the number of independent coefficients decreases to two. 


\begin{tabular}{|c|c|c|c|}
\hline System & $\begin{array}{c}\text { Characteristic } \\
\text { Symmetry }\end{array}$ & $\begin{array}{l}\text { Number of } \\
\text { independent } \\
\text { coefficients }\end{array}$ & $\begin{array}{c}\text { Tensor referred to axes } \\
\text { in the conventional- } \\
\text { orientation }\end{array}$ \\
\hline Cubic & 4 3-fold axes & 1 & {$\left[\begin{array}{lll}\alpha & & \\
& \alpha & \\
& & \alpha\end{array}\right]$} \\
\hline $\begin{array}{l}\text { Tetragonal } \\
\text { Hexagonal } \\
\end{array}$ & $\begin{array}{ll}1 & 4 \text {-fold axes } \\
1 & 6 \text {-fold axes } \\
\end{array}$ & \multirow[t]{2}{*}{2} & \multirow{2}{*}{$\alpha_{1}$} \\
\hline Trigonal & 13 -fold axes & & \\
\hline Orthorhombic & $\begin{array}{c}3 \text { mutually } \\
\text { perpendicular 2- } \\
\text { fold axes; no axes } \\
\text { of higher order }\end{array}$ & 3 & {$\left[\begin{array}{lll}\alpha_{1} & & \\
& \alpha_{2} & \\
& & \end{array}\right.$} \\
\hline Monoclinic & 12 -fold axes & 4 & {$\left[\begin{array}{lll}\alpha_{1} & & \alpha_{13} \\
& \alpha_{2} & \\
\alpha_{13} & & \alpha_{33}\end{array}\right]$} \\
\hline Triclinic & $\begin{array}{l}\text { A center of } \\
\text { symmetry or no } \\
\text { symmetry }\end{array}$ & 6 & {$\left[\begin{array}{lll}\alpha_{11} & \alpha_{12} & \alpha_{13} \\
\alpha_{12} & \alpha_{22} & \alpha_{23} \\
\alpha_{13} & \alpha_{23} & \alpha_{33}\end{array}\right]$} \\
\hline
\end{tabular}

Table 1: Relation between crystal symmetry and the thermal expansion tensor $\alpha$, specified with respect to the principal directions (i.e. conventional orientations) of the crystals in cubic, tetragonal, hexagonal, trigonal, and orthorhombic systems. Crystals in a monoclinic system have only one fixed principal axis, i.e. the direction of 2-fold axis (with respect to the $x_{2}$ direction). Crystals in triclinic system have no fixed principal axis, thus the $\alpha$ tensor is expressed as a general form in the last column. Groups without higher-order axes denote groups containing only 2 -fold axes, mirror planes, and inversion center, e.g. orthorhombic, monoclinic, and triclinic systems. (Nye, 1985)

The last column of Tab. 1 illustrates the CTE tensor of the corresponding crystal having its principal directions aligned along the axes defining the reference coordinate system of the crystal, i.e. $x_{1}, x_{2}$, and $x_{3}$. In this case, the CTE of a trigonal crystal, taken here as example, can be simply expressed as 


$$
\alpha_{i j}=\left[\begin{array}{lll}
\alpha_{1} & & \\
& \alpha_{1} & \\
& & \alpha_{3}
\end{array}\right] \text {, }
$$

where $\alpha_{1}$ and $\alpha_{3}$ refer to the $\mathrm{CTE}$ along the principal directions. If the reference axes are rotated with respect to the principal directions, the components of $\boldsymbol{\alpha}$ can be just obtained by applying a transformation law for second-rank tensor, through the matrix, $\left(l_{i j}\right)$, such as:

$$
\alpha_{m n}^{\prime}=l_{m i} l_{n j} \alpha_{i j}
$$

where the CTE tensor $\alpha_{m n}^{\prime}$ refers to the coordinate system after transformation, and $l_{m i}$ and $l_{n j}$ represent the components in the transformation matrix $\left(l_{i j}\right)$. The CTE value in any specific direction can then be evaluated through:

$$
\alpha=\alpha_{i j} l_{i} l_{j}
$$

where $l_{i}$ and $l_{j}$ are the direction cosines of the original axes with respect to the new rotated axes. By analogy, the symmetry systems reported above can be readily transferred to assess the CTE of dual-material lattices built from tetrahedral building blocks. In particular during the assembly of building blocks, the symmetrical arrangement of tetrahedra in a unit cell can be chosen to match that of a point group of a crystal. Fig. 5a and b show an example for a trigonal system with a 3fold axes. The screw geometry illustrates that this system relies on a 3-fold axes in which a single geometry is replicated twice through rotation about the axis at $2 \pi / 3$ intervals. By analogy, Fig. $5 \mathrm{c}$ shows that the trigonal system can be enforced to assemble 4 TNs. The result is the repeating unit in Fig. 5d, where three TNs form the base of the 3D lattice, and upon their apexes rests the fourth one. All the building blocks have 3-fold axes of symmetry, which is also the rule of symmetry of their spatial arrangement. Hence, the correspondence between the symmetry 
relationship of the building blocks within the unit cell and that of the crystal system used to assemble them, makes the CTE tensor of the overall lattice feature physical characteristics identical to those of a trigonal crystal. For the lattice in Fig. 5d, the CTE in Eq. (11) is defined by only two independent coefficients, i.e. $\alpha_{1}$ and $\alpha_{3}$, with only $\alpha_{3}$ having CTE tunability. Hence the unit cell holds a unidirectional CTE tunability along the principal direction $x_{3}$. Therefore, the calculation of the CTEs along the principal directions reduces to the mere appraisal of the $\mathbf{M}$ and $\mathbf{N}$ vectors of its building block, and the CTEs in any other directions can be obtained via Eq.

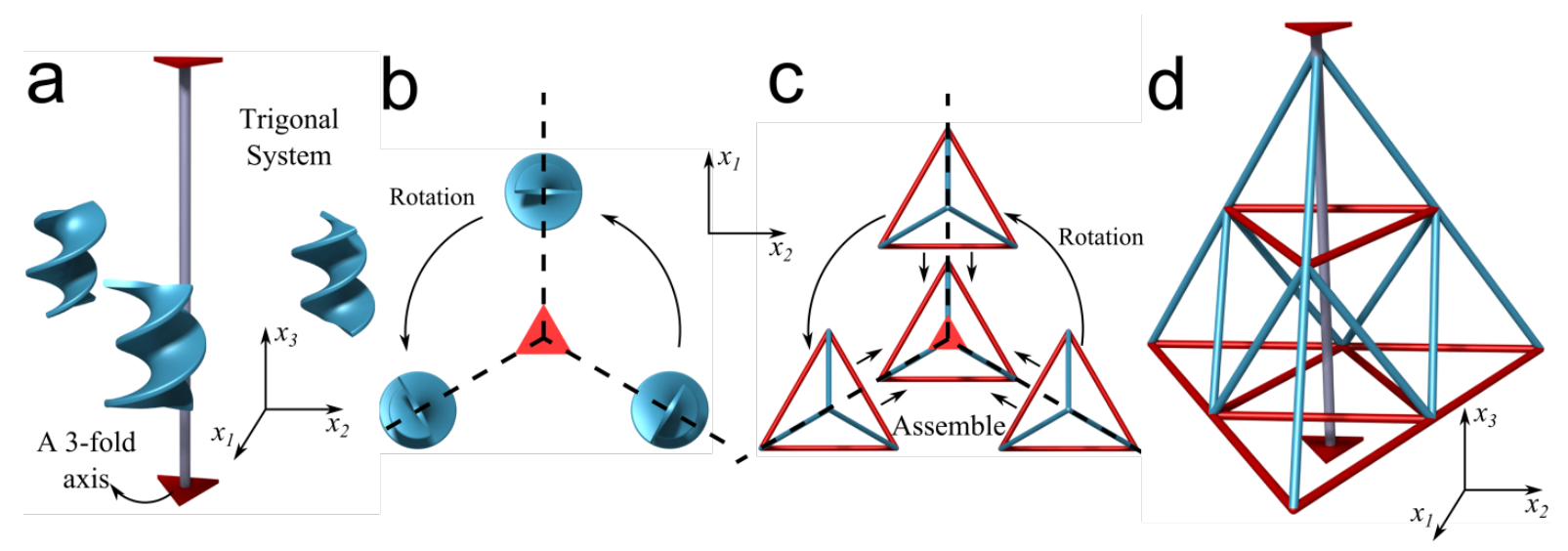

Figure 5: Trigonal crystallographic symmetry group applied to the generation of a spatial bi-material lattice with two independent coefficients, $\alpha_{1}$ and $\alpha_{3}$, of the CTE tensor: (a) screw geometry illustrating the 3-fold axes of symmetry with its top view shown in (b), (c) top view of building blocks assembled with 3-fold axes, and (d) axonometric view of the resulting unit cell.

The simple example above focusing on one symmetry system has demonstrated how the crystallographic point groups can guide the relative arrangement of building blocks in the repeating unit. This is just one, among others, of the possible systems listed in Tab. 1. When one of these symmetry systems is enforced to arrange building blocks in a unit cell, then a direct correlation emerges between the geometric symmetry of the building block and the symmetry of the elements of its CTE tensor. The elements in the CTE tensor that are independent represent the CTEs of the overall lattice in the principal directions, which can be evaluated via $\mathbf{M}$ and $\mathbf{N}$ 
vector analysis. This is shown in the following sections, where a crystal symmetry system is used to assemble building blocks into a repeating unit that can satisfy prescribed CTE requirements.

\subsubsection{Unidirectional CTE Requirement}

There is theoretically an infinite number of unit cells that can be proposed to meet the unidirectional requirement of CTE. Fig. 6a, b, and c show three of them, each constructed with its own building block and a specific relation of symmetry, but all have unidirectional CTE tunability in the vertical direction $\left(x_{3}\right)$.

The first unit cell in Fig. 6a consists of eight TL-1 building blocks assembled via reflection and rotational transformations to make each adjacent block appear in an upside-down position. In this unit cell, there are only three mutually perpendicular 2-fold axes with an inversion center but no axes of higher order, e.g. 3-fold or 4-fold axes; hence, the unit cell has an orthorhombic symmetry (Fig. 6g). All ML vectors of TL-1s in Fig. 6a are parallel with the vertical direction and therefore the CTE-tunable mechanism of TL-1 governs the thermal deformation of the unit cell in the vertical direction. As revealed in Tab. 1, the CTE tensor of an orthorhombic unit cell has three independent components with values equivalent to the principal CTEs. Although the overall CTE is orthotropic, the entire lattice shows unidirectional CTE tunability with magnitude of $\alpha_{3}=\alpha_{z, T L-1}$ (Eq. (8)) that is tunable in the vertical direction only. Along the other two principal directions $\alpha_{1}=\alpha_{s 1}$ and $\alpha_{2}=\alpha_{s 2}$, the periodic lattice can uniformly expand with the CTE of its components. The second unit cell shown in Fig. $6 \mathrm{~b}$ is structured with eight TL-2 building blocks via only rotation around, and translation along, the vertical direction. This unit cell has a 4-fold axis with a middle mirror plan, and thus classed as tetragonal symmetry. In its CTE tensor (Tab. 1), there are only two independent coefficients: $\alpha_{1}=\alpha_{s 1}$ and $\alpha_{3}=\alpha_{z, T L-2}$ (Eq. (9)). This unit cell, although obtained with symmetry relationship different from that of the previous model, still 
keeps all ML vectors of the building blocks along the vertical direction and therefore results in a unidirectional CTE controllable in the vertical direction. The unit cell in Fig. 6c uses the TN tetrahedron, which has a trigonal symmetry (Fig. 5b and Fig. 6i). As discussed above, the only two independent coefficients are $\alpha_{1}=\alpha_{s 1}$ and $\alpha_{3}=\alpha_{z, T N}$ (Eq. (10)), thereby demonstrating unidirectional CTE tunability.

The three concepts in Fig. 6 feature dissimilar symmetry systems constructed via different affine transformations, however, they all keep the $\mathbf{M}$ vectors in the CTE tunable direction along direction $x_{3}$. The direction $x_{3}$ of each concept follows the direction of the 2-fold, 3 -fold, and 4fold axes, which are principal directions. Each unit cell, therefore, inherits the CTE tunability of its building block in the principal direction $x_{3}$. Fig. 6p, $\mathrm{q}$, and $\mathrm{r}$ illustrate their omnidirectional CTEs in a spherical coordinate system, from which a pertinent symmetry in thermal expansion appears for each case. The direction with the lowest CTE (dark blue in the legend), i.e. a single vertical axis for each unidirectional concept, is the principal direction of CTE tunability. No CTE tunability is viable along the other principal directions, i.e. plane $x_{1}-x_{2}$.
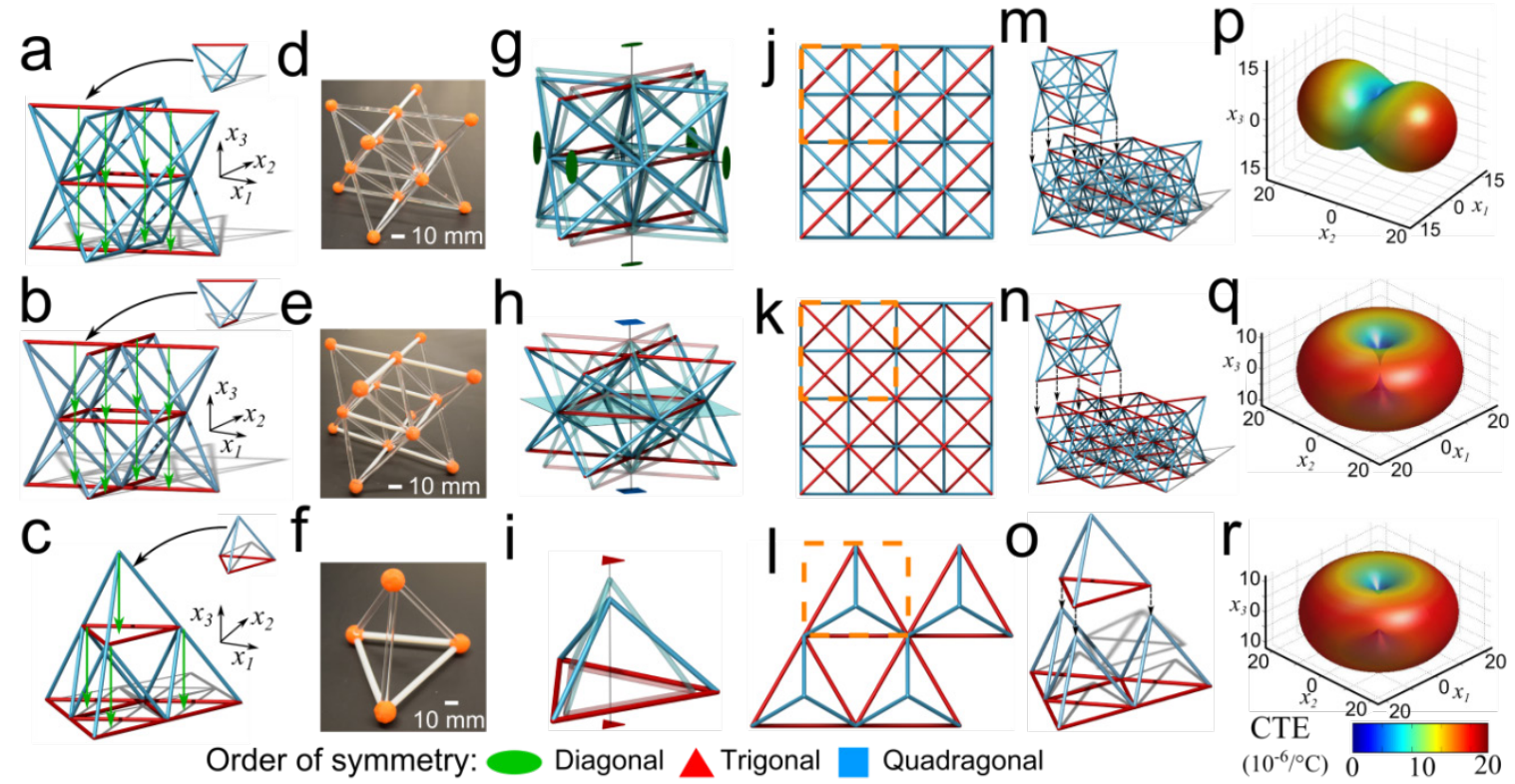

Figure 6: Unit cells with unidirectional CTE tunability: (a) TL-1 concept, (b) TL-2 concept, and (c) TN 
concept. Green arrows represent the ML vectors of the building blocks, all oriented along the $x_{3}$ direction. (d), (e), and (f) are images of their physical ball-and-stick models. (g), (h), and (i) show the deformed and undeformed concepts with the main characteristic symmetry of the building block arrangement. The order of rotational symmetry is given by one of the three graphical symbols: a cusped oval, a triangle, and a square. (j), (k), and (l) are the top view of the unit cell assembly. (m), (n), and (o) show an axonometric view of the unit cell assembly. (p), (q), and (r) show CTE magnitude plotted in polar coordinate system with respect to the principal directions, i.e. $x_{1}, x_{2}$, and $x_{3}$; the semi-axes of each CTE ellipsoid are the CTE coefficients, $\left|\alpha_{1}\right|$, $\left|\alpha_{2}\right|$, and $\left|\alpha_{3}\right|$ of the corresponding unit cell.

\subsubsection{Transverse isotropic CTE Requirement}

Fig. 7a, b, and c show three unit cells, each assembled with its own building block but all have trigonal symmetry to meet transverse isotropic CTE requirement. In the first example (Fig. 7a), low-CTE stationary lines of six TL-1s form the inner core with two sets of triadic building blocks at the same altitude $\left(x_{3}\right)$ arranged with equal angular spacing. On the middle mirror plane (Fig. 7g), three additional low-CTE bars are added, combined with the six low-CTE stationary lines, forming a central triangular bipyramid. This operation, considered along with the periodic tessellation in Fig. $7 \mathrm{~m}$, is enforced to lock any mechanism as well as to make the unit able to perform as a pin-jointed structure. As shown in Fig. 7g, the unit cell consists of 3-fold rotational symmetry, analogous to the trigonal crystal, it has only two independent components in the CTE tensor, $\alpha_{1}$ and $\alpha_{3}$. All TL-1 ML vectors deviate outward from the vertical direction by an identical deviation angle, $\gamma\left(\gamma \in\left(0^{\circ}, 90^{\circ}\right)\right.$ with $\gamma=75^{\circ}$ in Fig. 7a), which, together with the skew angle and component CTEs, controls the horizontal effective CTE with in-plane isotropic CTE tunability $\left(\alpha_{1}=\alpha_{2}\right)$. In the out-of-plane principal direction, i.e. $x_{3}$, if the tessellation of unit cells (shown in Fig. $7 \mathrm{j}$ and $\mathrm{m}$ only along plane $x_{1}-x_{2}$ for visual simplicity) allows connection only at the peaks of the triangular bipyramid, there is no CTE tunability, resulting in $\alpha_{3}=\alpha_{s 2}$. Thus, the overall effective CTEs of the unit cell in Fig. 7a are expressed as: 


$$
\left\{\begin{array}{c}
\alpha_{1}=\alpha_{2}=\frac{\alpha_{s 2} \cos \theta \tan ^{-1} \gamma+\left[\alpha_{s 2}-\left(\alpha_{s 1}+\alpha_{s 2}\right) \cos ^{2} \theta\right] / \xi}{\cos \theta \tan ^{-1} \gamma+\xi} \\
\alpha_{3}=\alpha_{s 2}
\end{array}\right.
$$

where $\xi=\left(1-2 \cos ^{2} \theta\right)^{1 / 2}$.

Similarly, for the second example in Fig. 7b, TL-1s are replaced by TL-2 building blocks to construct a unit cell with transverse isotropic controllable CTE. A triangular bipyramid core is constructed via six high-CTE stationary lines and three high-CTE bars added in the middle mirror plane. All TL-2s have an identical $\gamma\left(\gamma \in\left(0^{\circ}, 90^{\circ}\right)\right.$ with $\gamma=75^{\circ}$ in Fig. $\left.7 \mathrm{~b}\right)$. The symmetry relationship of the building blocks within the unit cell in Fig. $7 \mathrm{~b}$ is identical to that of TL-1 concept (Fig. 7a), and the unit cell has thermal expansion behavior expressed as:

$$
\left\{\begin{array}{c}
\alpha_{1}=\alpha_{2}=\frac{\alpha_{s 1} \cos \theta \tan ^{-1} \gamma+\left(\alpha_{s 2}-2 \alpha_{s 1} \cos ^{2} \theta\right) / \xi}{\cos \theta \tan ^{-1} \gamma+\xi} \\
\alpha_{3}=\alpha_{s 1}
\end{array} .\right.
$$

The third example in Fig. 7c shows that TN building blocks can also be manipulated to construct a unit cell with transverse isotropic CTE. Six TNs deviate from the vertical direction by $\gamma$ $\left(\gamma \in\left(0^{\circ}, 90^{\circ}\right]\right.$ with $\gamma=90^{\circ}$ in Fig. $\left.7 \mathrm{c}\right)$ and round up their high-CTE base members, together with the three high-CTE bars added on the middle mirror plane, to form the edges of a central polyhedron. Fig. 7 i shows the unit cell in a 3 -fold rotational symmetry, analogous to a trigonal crystal, resulting in only two independent components in the CTE tensor, $\alpha_{1}$ and $\alpha_{3}$. Within the horizontal plane, the concept has in-plane isotropic CTE tunability $\left(\alpha_{1}=\alpha_{2}\right)$. The out-of-plane tessellation (along plane $x_{1}-x_{2}$ in Fig. 7o) reveals a mere connection via the high-CTE cores, yielding $\alpha_{3}=\alpha_{s 1}$ with no CTE tunability in the vertical direction. For this reason, the thermal 
expansion behavior of the unit cell in Fig. 7c is expressed by:

$$
\left\{\begin{array}{c}
\alpha_{1}=\alpha_{2}=\frac{\left(3 \alpha_{s 2}-4 \cos ^{2} \theta \alpha_{s 1}\right) / \zeta+\alpha_{1} \cos \theta}{2 \sqrt{3} \cos ^{2} \theta+\zeta} \\
\alpha_{3}=\alpha_{s 1}
\end{array}\right.
$$

where $\zeta=\left(3-4 \cos ^{2} \theta\right)^{1 / 2}$.
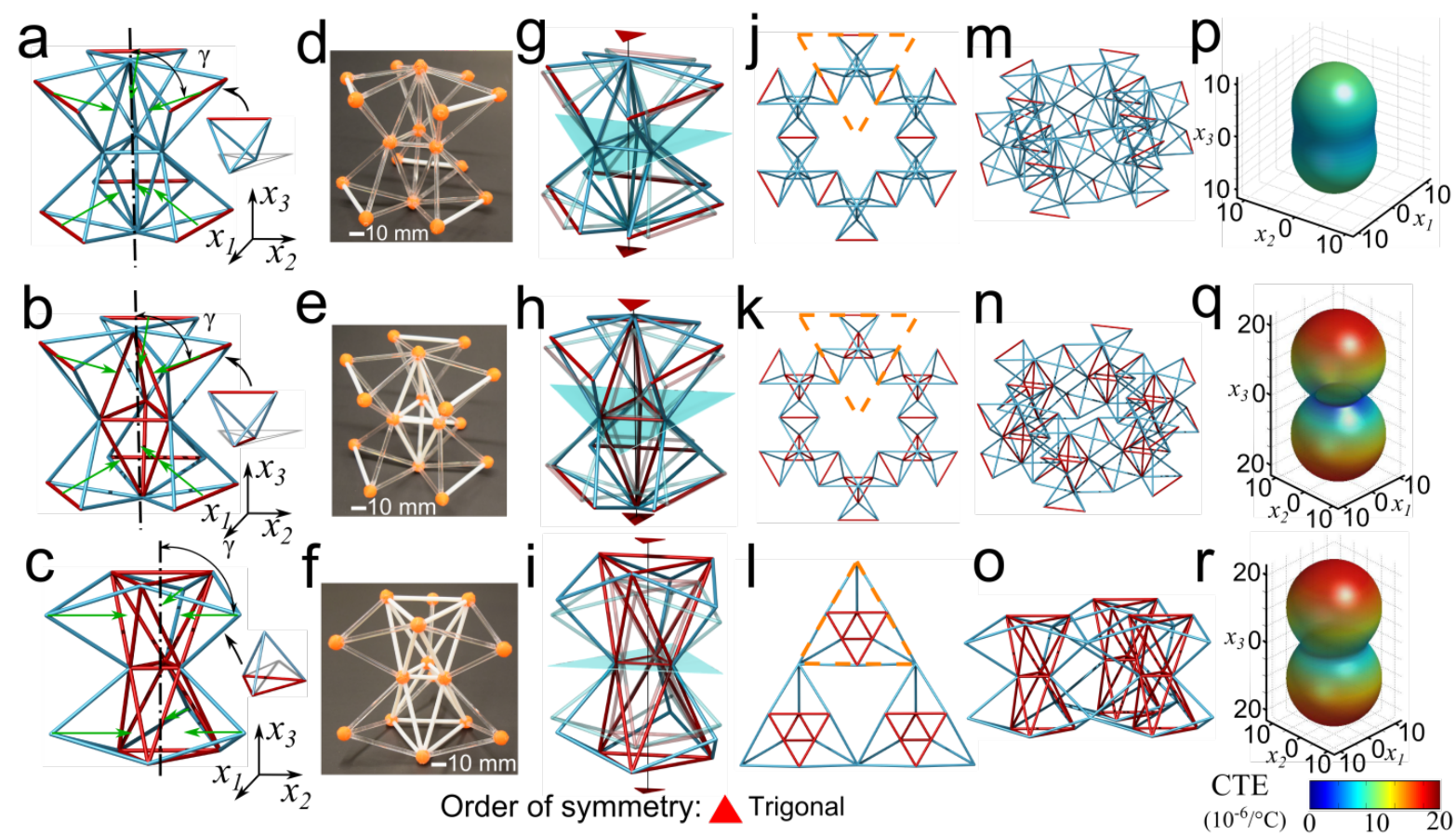

Figure 7: Low-CTE unit cells with transverse isotropic CTE tunability: (a) TL-1 concept, (b) TL-2 concept, and (c) TN concept. Green arrows represent the ML vectors of the building blocks. (d), (e), and (f) are images of their physical ball-and-stick models. (g), (h), and (i) show the deformed and undeformed concepts with main characteristic symmetry of the building block arrangement. The order of three-fold rotational symmetry is given by the triangle symbol. (j), (k), and (l) the top view of the unit cell assembly; (m), (n), and (o) show an axonometric view of the unit cell assembly. (p), (q), and (r) show CTE magnitude plotted in polar coordinate system with respect to the principal directions, i.e. $x_{1}, x_{2}$, and $x_{3}$; the semi-axes of each CTE ellipsoid are the CTE coefficients, $\left|\alpha_{1}\right|,\left|\alpha_{2}\right|$, and $\left|\alpha_{3}\right|$ of the corresponding unit cell.

We remark that the top views shown in Fig. $7 \mathrm{j}, \mathrm{k}$, and 1 are the $3 \mathrm{D}$ analogues of previous $2 \mathrm{D}$ concepts (Wei et al., 2016). The assembly of other spatial unit cells can be inspired by other 2D cell topologies in the literature (Miller et al., 2008; Steeves et al., 2007). Furthermore, as shown 
in the examples of Appendix A, the vertical direction $\left(x_{3}\right)$ can also possess CTE tunability by decreasing the deviation angle, $\gamma$. Fig. $7 \mathrm{p}, \mathrm{q}$, and $\mathrm{r}$ show the omnidirectional CTEs of the concepts in a spherical coordinate system. A central horizontal plane circularly shaped (dark blue in Fig. $7 p$ to $r$ ) shows principal directions with CTE tunability for each transverse isotropic concept. Contour plot symmetry in Fig. $7 \mathrm{p}$ to $\mathrm{r}$ also reflects the symmetry in thermal expansion, such as no CTE tunability is viable in the vertical direction.

\subsubsection{Isotropic CTE Requirement}

Fig. 8 shows three unit cells, one for each row, possessing isotropic controllable CTE, with TL-1s, TL-2s, and TNs as their building blocks respectively. A regular octahedron core is constructed by either stationary lines of TL building blocks or high-CTE base triangles of TNs. This monolithic core has a uniform thermal expansion in all directions and connects all building blocks via identical distance to the center of each unit cell for given angular space. All the unit cells have a cubic envelope as the unit cell domain. Along the four body diagonal directions of the cubic domain, four 3-fold axes can be found for each unit cell (Fig. 8g, h, and i), thus showing a cubic symmetry. Analogous to the cubic crystal, there is only one independent coefficient in the CTE tensor of each concept, and therefore each unit cell has identical thermal expansion in all directions. Hence, the tessellated periodic lattices, with all unit cells connected by either stationary nodes or stationary lines (Fig. $8 \mathrm{j}$ to o), feature a CTE that is isotropic with adjustable magnitude given by:

$$
\begin{array}{ll}
\alpha_{I s o-T L-1}=\frac{\alpha_{s 2} \cos \theta+\left[\alpha_{s 2}-\left(\alpha_{s 1}+\alpha_{s 2}\right) \cos ^{2} \theta\right] / \xi}{\cos \theta+\xi}, & \text { for Fig. 8-a; } \\
\alpha_{I s o-T L-2}=\frac{\alpha_{s 1} \cos \theta+\left(\alpha_{s 2}-2 \alpha_{1} \cos ^{2} \theta\right) / \xi}{\cos \theta+\xi}, & \text { for Fig. 8-b; }
\end{array}
$$




$$
\alpha_{I s o-T N}=\frac{\alpha_{s 1} \sqrt{2} \cos \theta+\left(\alpha_{s 2}-4 / 3 \alpha_{s 1} \cos ^{2} \theta\right) / \zeta}{\sqrt{2} \cos \theta+\zeta},
$$

The unit cells examined in Fig. 8 are some among many other possibilities. Fig. 2 d shows another example of a unit with isotropic CTE tunability, previously studied in the literature (Steeves et al., 2007); this cell has an analogous spatial structure of carbon atoms in a diamond, i.e. cubic symmetry. More examples are shown in Appendix A. Fig. $8 p$ to $r$ indicate the omnidirectional CTEs of the concepts in a spherical coordinate system. Evidently, the isotropic CTEs are described by monocoloured spheres, reflecting the symmetry in thermal expansion. The principal directions can be randomly selected within the entire spherical space, yet obtaining the identical lowest CTE for each unit cell.

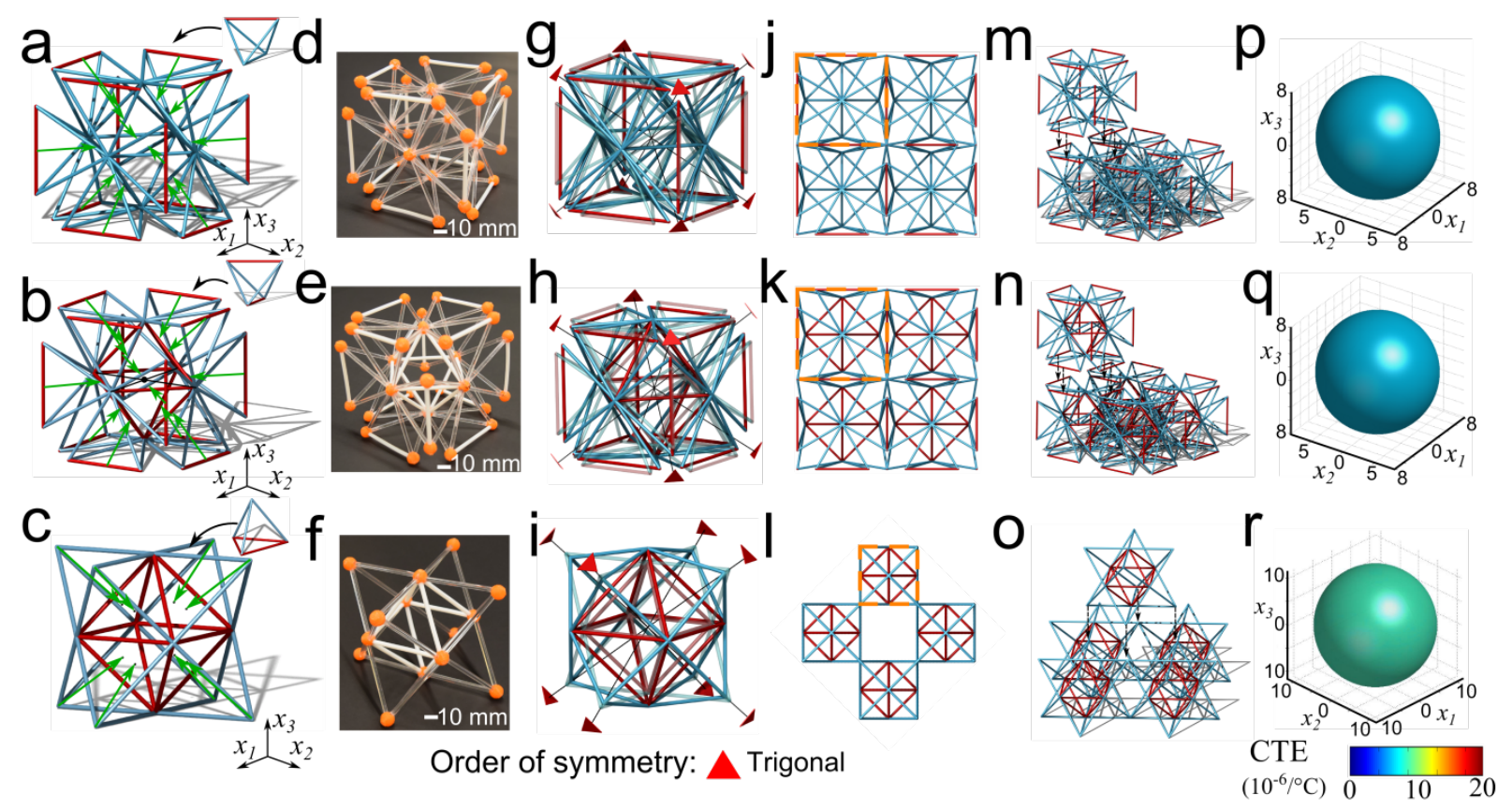

Figure 8: Unit cells with isotropic CTE tunability: (a) TL-1 concept, (b) TL-2 concept, and (c) TN concept. Green arrows represent the ML vectors of the building blocks. (d), (e), and (f) are images of their physical ball-and-stick models. (g), (h), and (i) show the deformed and undeformed concepts with the main characteristic symmetry of the building block arrangement. The order of three-fold rotational symmetry is given by the triangle symbol. (j), (k), and (l) are the top view and assembly. (m), (n), and (o) show an axonometric view of the unit cell assembly. (p), (q), and (r) show CTE magnitude plotted in polar coordinate system with respect to the principal directions, i.e. $x_{1}, x_{2}$, and $x_{3}$; the semi-axes of each CTE ellipsoid are the 
CTE coefficients, $\left|\alpha_{1}\right|,\left|\alpha_{2}\right|$, and $\left|\alpha_{3}\right|$ of the corresponding unit cell.

\subsubsection{Geometrical constraints of compound unit cells}

For the nine concepts illustrated in Fig. 6, 7 and 8, the effective CTEs in the CTE tunable direction can be programmed through a change of the skew angle, $\theta$, of the building block. The range of $\theta$ is restricted by certain values that preserve the tetrahedral shape of the building block, as well as avoid causing collision between adjacent unit cells during thermal expansion. For concepts with transverse isotropic CTE, the range of $\theta$ is also governed by $\gamma$. Tab. 2 shows the allowable range of $\theta$ for each cell topology with $\varphi$ representing the packing factor of the lattice. A packing factor correlates to a given tessellation. For example, a packing factor of $100 \%$ is shared by the unit cells shown in Fig. 6m, n, and o. In contrast, for the unit cells with isotropic CTE given ranges of their skew angles result in tessellations with lower packing factors, such as $50 \%$ for the tessellation shown in Fig. $80\left(\theta=60^{\circ}\right)$. Differences in the packing factor are controlled by the requirement of the inner polyhedron, e.g. octahedron in Fig. 8c, to thermally expand without touching adjacent cells.

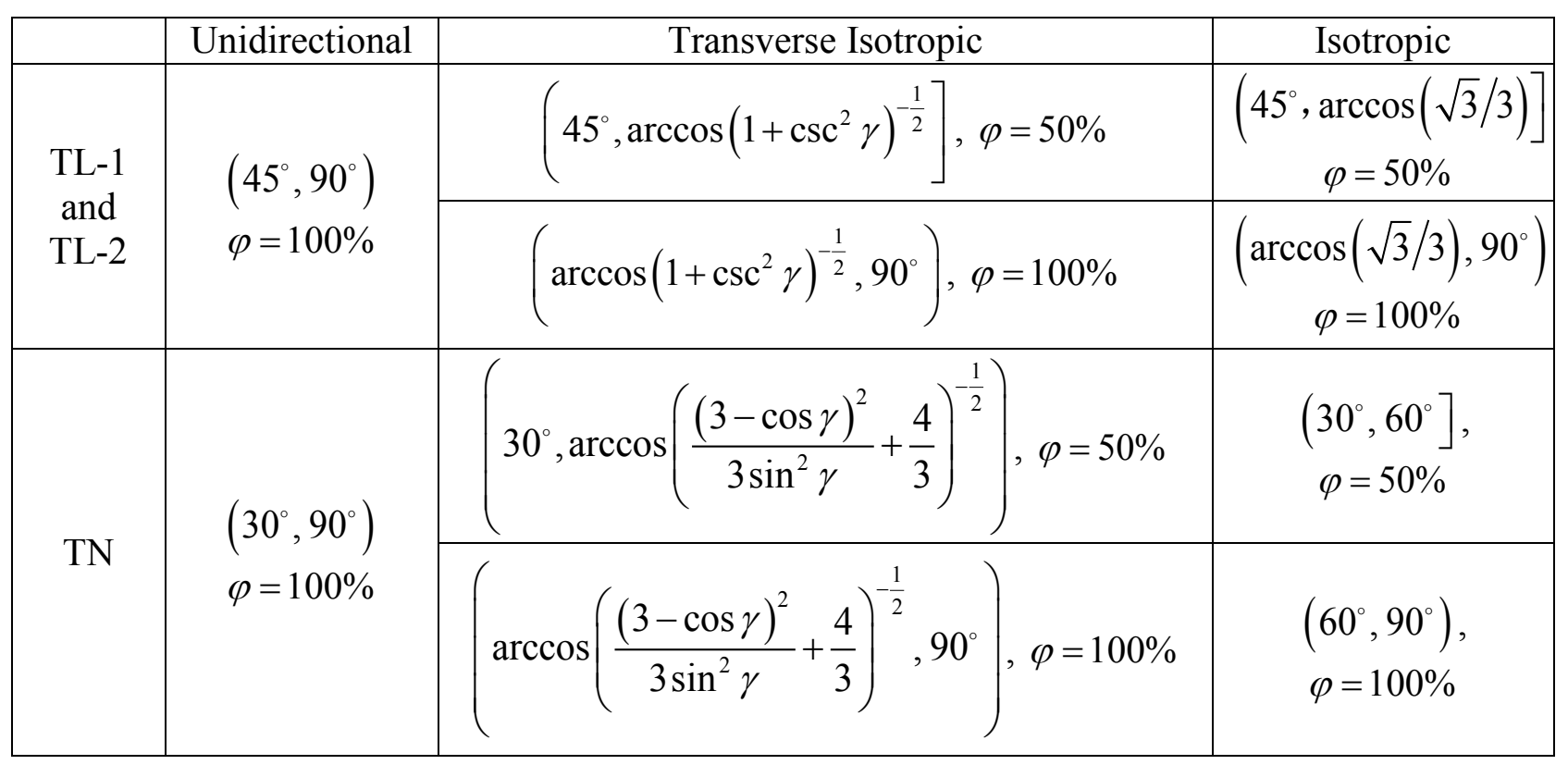

Table 2: Admissible range of the skew angle, $\theta$, for the building blocks of the concepts visualized in Fig. 6 , 7 
and 8. $\gamma$ and $\varphi$ represent the deviation angle and packing factor respectively.

\subsubsection{Summary points for prescribing thermal expansion in spatial lattices}

There are plentiful ways to construct 3D lattices from tetrahedral building blocks. Below, the key notions explained above are summarized to help assemble tetrahedron-based lattices that can meet given magnitude and directional requirements of thermal expansion. The distinguishing features are the building blocks with the definition of their ML and TD vectors, and the symmetry construction operations used to assemble them in the repeating unit.

- Building blocks with their thermal expansion. The bi-material tetrahedron is the smallest building block chosen here for generating a periodic 3D lattice. Nine relevant material permutations are available (Fig. $3 \mathrm{~b}$ to j), among which the most practical are those with stationary lines, TL-1 and TL-2, and with stationary node, TN. For each of them, the material length vector, ML, and the thermal displacement vector, TD, provide a visual and handy description of the magnitude and direction of their thermal expansion. They assist in meeting requirements of given CTE magnitude and closed form expressions are given in section 2.2 to quantify them.

- Unit cell construction for prescribed CTE behaviour. Building blocks can be used to assemble complex compound units either with unidirectional CTE tunability (e.g. Fig. 6a) or with a number of tunable CTE directions (e.g. Fig. 8c), as prescribed by the application requirements. The crystal systems in Tab. 1 can assist their arrangement to satisfy directional requirements. If the symmetry relations of one crystal system transfer to the geometrical arrangement of building blocks in a unit cell, analogously the independent tensor components of that crystal system return into the CTE tensor of the overall lattice. Here, the tensor components of the unit cell represent the CTEs in the principal directions, either with or without CTE tunability, of the overall lattice. By following this strategy the 
overall thermal expansion of a spatial lattice built from compound units can be assessed through the ML and TD vectors of its constitutive building blocks (section 2.2).

In this summary, we also remark that unit cell construction should also meet tessellation requirements, such as those ensuring minimum static and kinematic determinacy, among others. If the assembly process leads to an unstable mechanism, additional members are necessary to lock in any mechanism, and additional struts can be inserted to match the CTE of its surrounding elements so as to avoid altering the direction(s) of CTE-tunability. In addition, because there are multiple tetrahedral building blocks and numerous relations of symmetry to choose from, the number of 3D tessellations capable of satisfying given CTE requirements can be countless. This work has presented nine of them (Fig. 6 to 8 ) obtained by implementing the scheme presented here, and the following section describes their proof-of-concept fabrication and thermal testing. Additional concepts showing the viability of the method are given in Appendix A.

\section{Fabrication and experimental validation}

\subsection{Component materials}

This section presents the fabrication and CTE testing of i) the building blocks, TN and TL-2, made from metallic constituents, and ii) the nine lattice concepts shown in Fig. 6 to 8, made from polymers. The six constituent materials selected are: A16061, Ti-6Al-4V, and Invar-36 as metallic constituents; and acrylic, Teflon ${ }^{\circledR}$ PTFE, and ABS as polymer constituents. Tab. 3 shows their relevant properties.

\begin{tabular}{|c|c|c|c|c|c|c|}
\hline Material & Al 6061 & Ti-6Al-4V & Invar-36 & Acrylic & PTFE & ABS \\
\hline Young's modulus (GPa) & 70.8 & 113.8 & 140.0 & 3.2 & 0.475 & 2.6 \\
\hline CTE $\left(\times 10^{-6} /{ }^{\circ} \mathrm{C}\right)$ & 23.0 & 11.5 & 1.5 & 67.0 & 123.0 & 94.5 \\
\hline
\end{tabular}

Table 3: Properties of solid materials for proof-of-concept fabrication (USDOD, 1998) 


\subsection{Engineering of specimens}

\subsubsection{Fabrication of building block samples}

Testing samples of building blocks were fabricated via pin-jointed metallic bars (Fig. 9a to d). Ti6Al-4V shafts (low-CTE) were cut and sanded to the desired length (Fig. 9b and d) with each end shaped into a crevice. A flake in the shape of a ring tongue terminal was fastened in the crevice of bars by interference fit and strengthened via administering epoxy glue (LePage Epoxy Gel, Henkel, Canada) to serve as a hinge axle sleeve for pinned joints. As shown in Fig. 9, A16061 shafts (high-CTE) are thicker (diameter of $6.4 \mathrm{~mm}$ ) than the Ti-6Al-4V bars (diameter of $3.2 \mathrm{~mm}$ ) so as to construct a stable base with through-holes drilled directly to serve as hinge axle sleeves. All the high CTE bars have given length of 50mm (Fig. 9b and d), and the length of low-CTE bars vary for modifying the skew angle at given values. Then the assembly of the different tetrahedral samples were completed using bolts, screw nuts and washers. Since only rotation

occurs and no bending moment appears at the joints, the tested CTE of pin-jointed building blocks are validated via closed-form equations in section 2.3 relying on the pin-jointed assumption. Furthermore, in the fabricated prototypes shown in Fig. 9, the length of the flakes (totalling about $12 \mathrm{~mm}$ at both ends), compared with the typical length of the shaft (about 40 to $55 \mathrm{~mm}$ ), is not negligible. Thus, the thermal effect of flakes on the overall effective CTE of lattices is taken into account to amend the analytical model as further explained in Appendix B.

\subsubsection{Fabrication of unit cell specimens}

To ease the understanding of the spatial arrangement of building blocks in all unit cells, we built ball-and-stick models as proof-of-concepts (Fig. 6 to 8). Connection balls (10 mm diameter) have several blind holes (with a $1.6 \mathrm{~mm}$ diameter and $2 \mathrm{~mm}$ depth) in different orientations on the surface (Fig. 9g) to connect sticks (Fig. 9e and f). All the spheres are 3D printed (printer: Original Prusa i3 MK2) by ABS. The sticks were laser cut into different lengths, all from 1.6-mm- 
diameter rods made from either PTFE (high-CTE) or acrylic (low-CTE). Both ends of the sticks were plugged into the blind holes of spheres and then rigidly fixed by resin glue (LePage Epoxy Gel, Henkel, Canada). The thickness of the resin adhesive layer was very thin making the thermal expansion of resin glue negligible. In contrast, the ABS connection ball had a non-negligible thermal expansion, and this deviation is considered in a later section validating experimental measures against theoretical and simulation results.
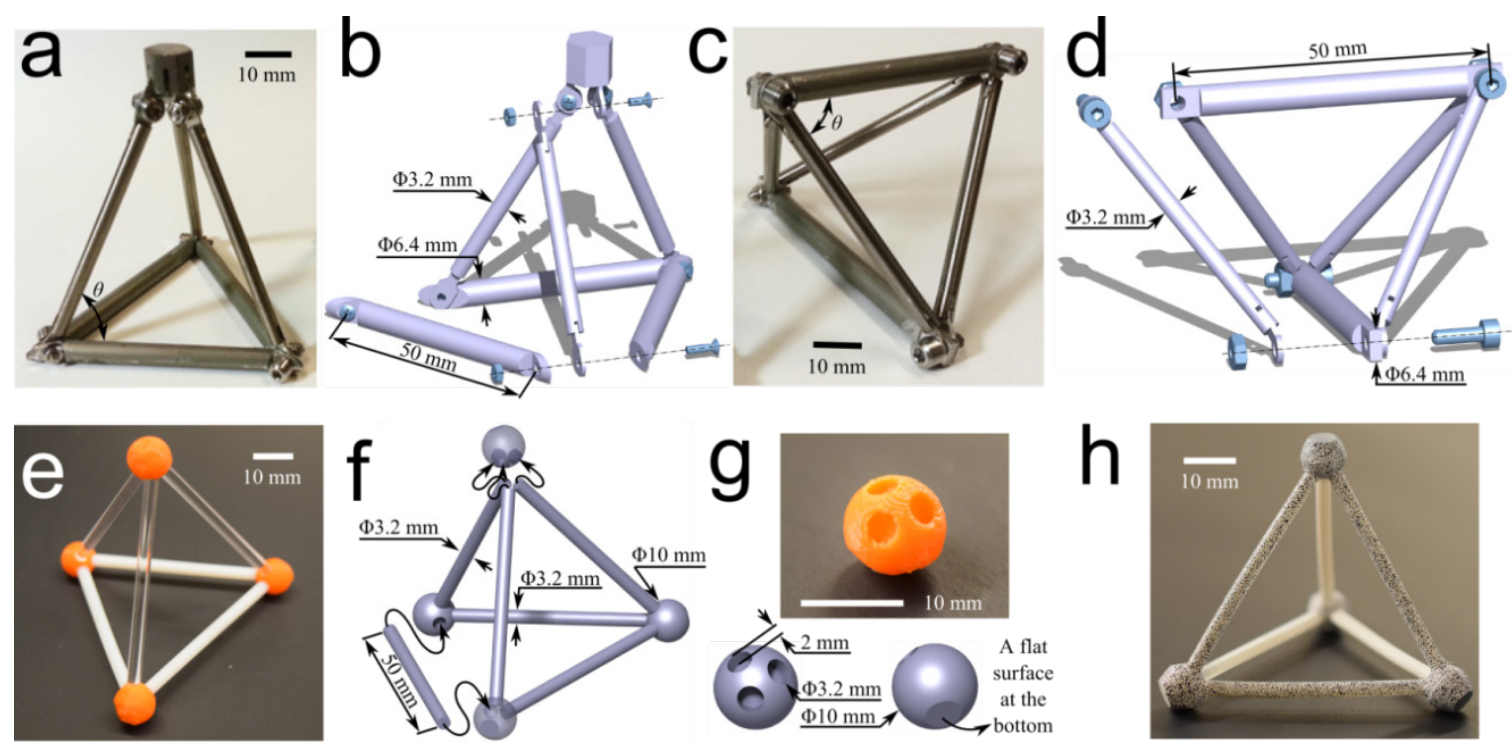

Figure 9: Testing samples: (a) TN (components: Al6061 and Ti-6Al-4V); (b) assembly drawing of a TN; (c) TL-2 (components: Al6061 and Ti-6Al-4V); (d) assembly drawing of a TL-2; (e) TN concept with rigid joints (components: acrylic and PTFE for bars and ABS for joints); (f) assembly drawing of proof-of-concepts with rigid joints; (g) ball for rigid joint (a flat surface at the bottom is used to ensure continuous contact with the carrier of the 3D printer); (h) testing sample with black and white pattern for DIC testing.

Moreover, since the CTE properties of the concepts here introduced are primarily dependent on the unit cell geometry besides the CTEs of the constituent solids, the principles can be applied to systems across a wide range of length scales, and hence fabricated with both conventional and advanced methods. Additive manufacturing is a viable method that can be effortlessly used to assemble in large volume bi-material lattices with smaller element size, as demonstrated by recent works appeared in the literature on this topic (Qu et al., 2017; Takezawa and Kobashi, 2017; Wang et al., 2016). 


\subsection{Experimental method}

Both tetrahedral building blocks (Fig. 9) and compound unit cells (Fig. 6 to 8) were experimentally investigated to validate their CTEs. For the former, 12 physical samples were tested to verify the respective theoretical models of TL-2 and TN building blocks (Eqs. (9) and (10) in section 2.2.1) with two dissimilar pair of material combinations and three different skew angles (see Appendix B for the images of the physical samples of the building blocks). The first material combination consisted of Al6061 and Ti-6Al-4V (i.e. Al/Ti), and the second consisted of Al6061 and Invar (i.e. Al/Invar). In all cases, Al was the high CTE material while Invar and Ti were the low CTE materials. The three skew angles of the building blocks for each material combination varied between $53^{\circ}$ to $65^{\circ}$ with approximately equal angle difference. For example, if the 12 samples were organized into four sets of three, the first set would consist of three TL-2 samples made from $\mathrm{Al} / \mathrm{Ti}$ with skew angles $53.5^{\circ}, 61.7^{\circ}$ and $63.7^{\circ}$, respectively. Besides building blocks, nine physical samples of the compound unit cells were tested, one for each of the concepts shown in Fig. 6 to 8 (see Appendix B for the images of the compound unit samples). The skew angles of all samples were set to $60^{\circ}$, and the CTEs along the principal directions were measured by assessing the relative thermal displacement of the connection balls.

3D DIC tests (schematic setup shown in Appendix B) were performed to measure the thermal displacement of all samples with randomly distributed black and white pattern painted on the surface (Fig. 9h). DIC testing consists of capturing images, before and after heating the sample within a heating chamber, at selected temperatures so that elongation can be measured. Testing temperature was monitored and managed from $25^{\circ} \mathrm{C}$ to $75^{\circ} \mathrm{C}$ (for polymer samples) or to $150^{\circ} \mathrm{C}$ (for metallic samples) through a PID (proportion-integration-differentiation) controller (CN7800, Omega, US). A data acquisition system (NI cDAQ 9174) was used to measure the temperature heterogeneity via collecting three thermocouples from different locations in the chamber. The 
temperature heterogeneity was regulated within $5 \%$ of the real-time temperature through the application of a rotational air fan. DIC system calibration ensured an epipolar projection error below 0.01 pixel, i.e. the average error between the position where a target point was found in the image and the theoretical position where the mathematical calibration model located the point. Two CCD (charge coupled device) cameras (PointGrey, Canada) were used to focus on an area of $240 \times 200 \mathrm{~mm}^{2}$ with a resolution of $2448 \times 2048$ pixel; based on the image resolution, any deformation smaller than $0.98 \mu \mathrm{m}(0.01$ pixel $)$ was merged with the epipolar projection error. Using the DIC correlation software, Vic-3D (Correlate Solution Inc.), virtual extensometers were placed on the reference image and tracked through the images to measure the displacement between pairs of pixel subsets. The thermal deformation field (Fig. 10) was obtained from the relative displacement between these pairs of subsets. The effective CTE was calculated from thermal strain and temperature change. Finally, the accuracy of the whole testing system was verified with CTE measurements of a solid material, A16061, taken from a commercial thermomechanical analyzer, TMA Q400 (TA Instrument, US). A comparison of their measured $\left(22.6 \times 10^{-6} /{ }^{\circ} \mathrm{C}\right)$ and DIC predicted mean CTE $\left(23.0 \times 10^{-6} /{ }^{\circ} \mathrm{C}\right)$ shows an error of $1.7 \%$. The epipolar projection error is at $0.98 \mu \mathrm{m}$, which governs the smallest measured CTE value of the samples, i.e. $0.27 \times 10^{-6} /{ }^{\circ} \mathrm{C}$. Hence, the low magnitude of these errors warrants the required accuracy for the DIC system used in this work.

\subsection{Experimental results}

The thermal testing results of both the building blocks and compound unit cells are here compared with results from either numerical analysis (Fig. 10) or closed-form expressions (Fig. 11). The role of component materials and skew angle on the effective CTE is emphasized. In addition, the effect of connection type, either pin-joint (building blocks) or rigid-joint (unit cells), is assessed to validate the pin-jointed assumption of the closed-form expressions derived for ideal 
building blocks and compound unit cells.

As shown in Fig. 10a to h, a TL-2 and a TN, both pin-jointed with similar $\theta$ and identical components of $\mathrm{Al} / \mathrm{Ti}$, are taken as representatives to illustrate the tunable thermal displacement that each tetrahedron can provide along the principal directions. The horizontal thermal displacement shown in Fig. 10 (a and b for TL-2 with $\theta=61.7^{\circ}$, e and f for TN with $\theta=63.3^{\circ}$ ) indicates that for both building blocks, the effective CTE is high and positive $\left(23 \times 10^{-6} /{ }^{\circ} \mathrm{C}\right)$, identical to that of the high-CTE constituent Al, i.e. no CTE tunability. In the vertical direction of TL-2 (Fig. 10c and d), the nearly alike light-green color on nodes used for CTE measurement (i.e. nodes B1 and B2 in Fig. 10d) indicates a near zero distance change. Thus, the effective CTE of TL-2 specimen in the vertical direction is almost vanishing $\left(3.70 \times 10^{-6} /{ }^{\circ} \mathrm{C}\right)$. In contrast, along the vertical direction of TN (Fig. 10g and h), the distance between the two corresponding nodes, D1 and D2 in Fig. 10h used for CTE measurement, increases during thermal expansion, which results in a positive effective $\mathrm{CTE}\left(8.42 \times 10^{-6} /{ }^{\circ} \mathrm{C}\right)$, a value below those of its constituents $\mathrm{Al}$ $\left(23 \times 10^{-6} /{ }^{\circ} \mathrm{C}\right)$ and $\mathrm{Ti}\left(11.5 \times 10^{-6} /{ }^{\circ} \mathrm{C}\right)$. Thus for similar skew angle and identical constituents, TL-2 can attain a lower CTE than TN. 

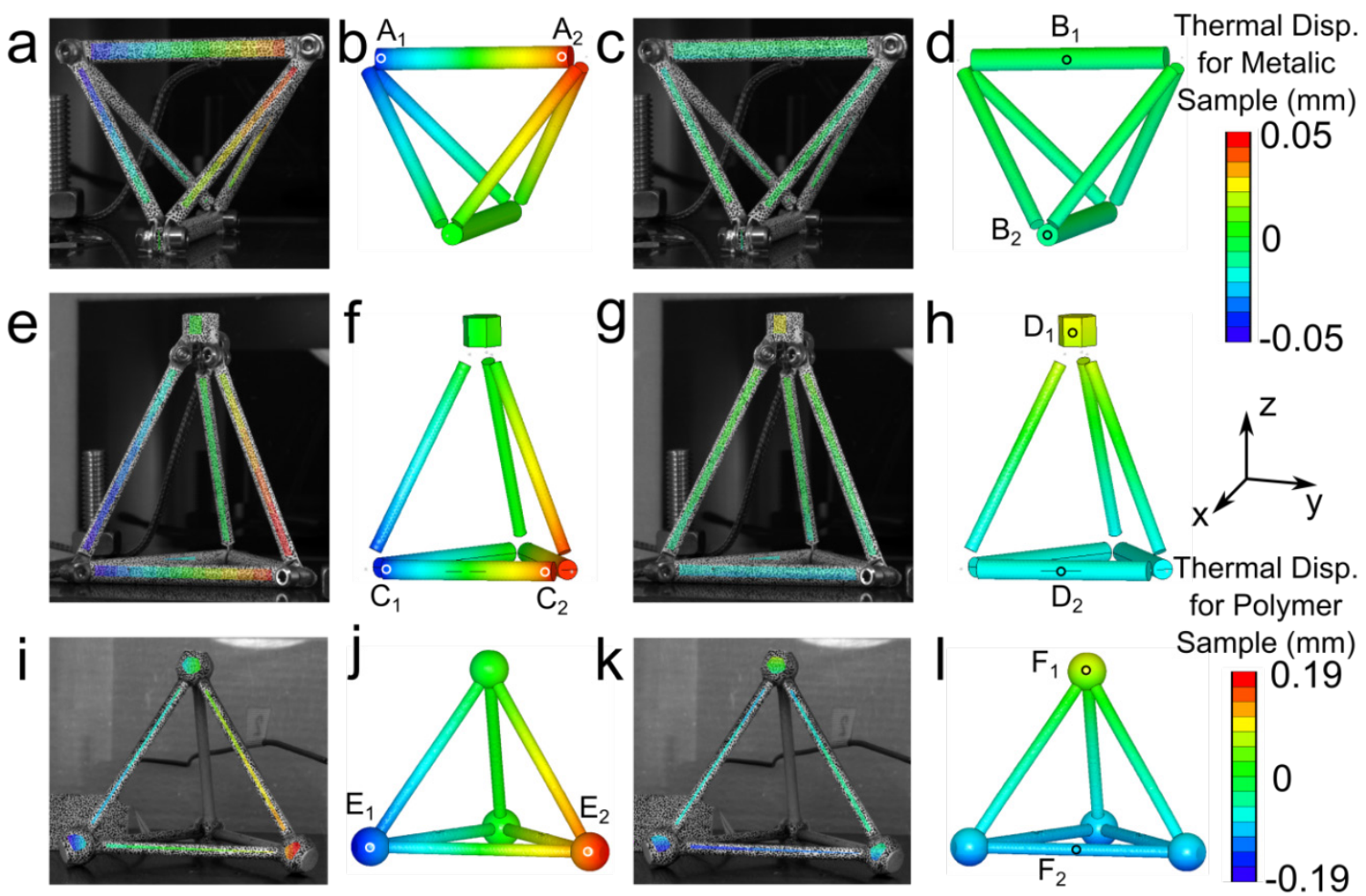

Figure 10: Thermal displacement distribution in a pin-jointed TL-2 ( $\left.\theta=61.7^{\circ}, \mathbf{A l} / \mathbf{T i}\right)$ measured experimentally (a and c), and computationally ( $b$ and $d$ ), in the horizontal and vertical directions, respectively. Thermal displacement distribution in pin-jointed $\mathbf{T N}\left(\theta=63.3^{\circ}, \mathbf{A l} / \mathbf{T i}\right)$ and rigid-jointed $\mathbf{T N}\left(\theta=60^{\circ}\right.$, acrylic/Teflon) tested (for pinned: $e$ and $g$; for rigid: $i$ and $k$ ) and computed numerically (for pinned: $f$ and $h$; for rigid: $j$ and $l)$ in the horizontal and vertical directions, respectively.

To assess the assumption of the unit cell connections, pin- versus rigid-joints, we compare the results shown in Fig. 10e to h, to those in Fig. 10i to 1 illustrating the tested thermal displacement distribution of a unit cell specimen (Fig. 6f). In both directions, the thermal distribution of the former made of $\mathrm{Al}$ and $\mathrm{Ti}$, parallel that of the latter, made of acrylic and PTFE, despite the difference in magnitude caused by each given pair of constituent materials.

Fig. 11a illustrates the impact of the skew angle, building block type, and component material on the effective CTE of building blocks. For all sets of samples, the CTE reduces significantly with decreasing skew angle, whereas for high values the CTE is less sensitive; nevertheless, CTE tuning across the range of skew angle here considered can be achieved for both TN and TL-2. In addition, for prescribed pair of materials, i.e. (Al/Ti) and (Al/Invar), the red and green curves 
show the CTE trend of TN with respect to the skew angle, and the blue and violet curves that of TL-2. The results show that the CTE of TL-2 can be tuned to a smaller value than that of TN, hence the $\triangle C T E$ of TL-2 is larger in the given range of skew angle. Another insight that can be gained from Fig. 11a pertains to the role of the CTE ratio of the constituents, i.e. $\lambda=\alpha_{s 1} / \alpha_{s 2}$. The greater the CTE distinction of the constituent solids $\left(\lambda_{\text {Al-Invar }} \approx 15.3\right.$ which is higher than $\lambda_{\mathrm{Al}-\mathrm{Ti}}=2.0$ ), the larger the CTE tunability of a building block. We also remark that the largest negative CTE a building block can reach in all experiments of this work is $-26.2 \times 10^{-6} /{ }^{\circ} \mathrm{C}$ (A1/Invar TL-2 with $\theta=54.1^{\circ}$ ) which is well below the CTE values of its components. 

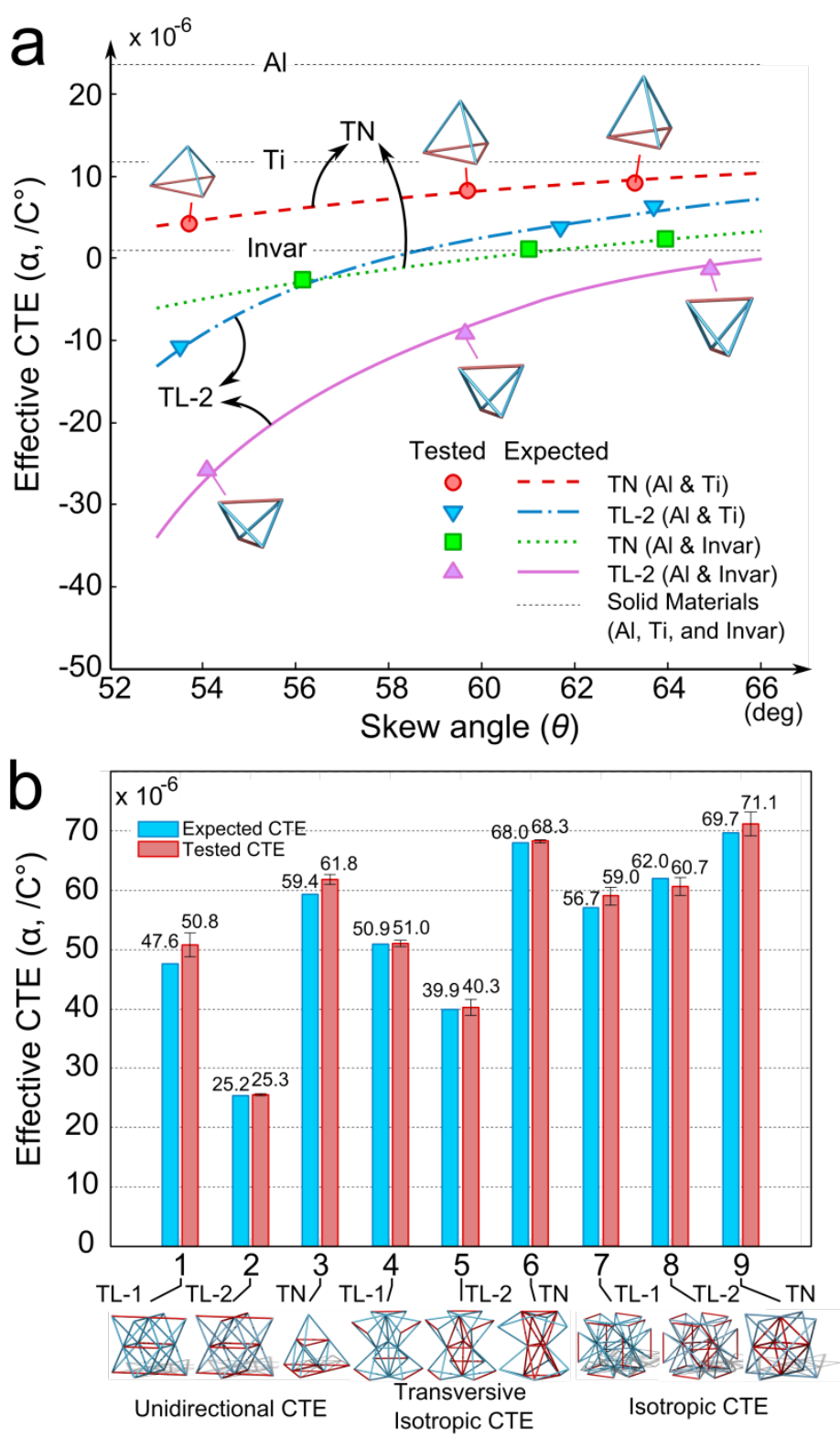

Figure 11: (a) Predicted curve and experimental results of effective CTE for Al/Ti and Al/Invar building blocks (TL-2 and TN) within a range of skewness, along with the CTE of the solid materials. (b) Predicted and experimental CTE results along the principal direction/s: vertical direction $\left(x_{3}\right)$ for the concepts with unidirectional CTE and principal directions within the plane $x_{1}-x_{2}$ for other concepts here examined.

Fig. $11 \mathrm{~b}$ compares the experimental (specimens with rigid-joints) and theoretical (closed-form expressions with pin-joints) results for the CTE of all the compound units. The relatively small errors associated with the testing results, which go as high as $6.3 \%$, validate the CTEs under pinand rigid-joint assumptions displaying small deviations, hence respecting the small deformation 
assumption. Another insight concerns the effective CTE of the building blocks here investigated. As shown in Fig. 11b, comparing the three concepts with unidirectional CTE tunability (i.e. specimens 1, 2, and 3 in Fig. 11b), specimens with stationary lines (TL-1 and TL-2) have smaller effective CTE values than that of specimens with stationary nodes. The conclusion up to this point is summarized as TL concepts have larger CTE tunability than TN concepts which also applies to the concepts with identical transverse isotropic or isotropic CTEs. The comparison between three concepts made of TL-1s (i.e. specimens 1, 4, and 7 in Fig. 11b), or other concepts made of the identical building block, show an increase in the effective CTE when the CTEtunable directionality increases from unidirectional (one principal direction) to transvers isotropic (two principal directions) and then to isotropic CTE (three principal directions). An increase of CTE-tunable directionality is accompanied by the presence and size increase of the monomaterial core of concepts. For example, unidirectional cells contain no monomaterial core, but transverseisotropic cells have small cores compared to isotropic cells' large cores. The presence and size of a monomaterial core make the effective CTEs increase with the size of the core. However, TL-2 concepts are more significantly affected by the high-CTE (red) cores than TL-1 concepts with low-CTE (blue) cores. Thus in Fig. 11b, with a triangular bipyramid core, specimen 4 has a larger CTE than specimen 5, while in contrast, specimen 7 has a smaller CTE than specimen 8 when an octahedron core is constructed for the isotropic concepts. The fabrication and testing method reported in this work can also be applied to building blocks with effective CTEs higher than that of the constituents (i.e. high-CTE cases as shown in Fig. 3) as well as concepts with more complex architecture.

\section{Mechanical properties}

To understand how a programmable CTE truss-system behaves as a bulk material with effective properties, we adopt here a classical continuum-based approach that relates the stress-strain 
behaviour of the unit cell to that of the global level. In doing so, we assume that the characteristic length of the unit cell is at least one or two orders of magnitude below the characteristic length of the truss-system. This scale separation between the global response and that of the unit cell allows us to calculate the effective properties through a continuum model (Arabnejad and Pasini, 2013). For three dual-material unit cells with unidirectional CTE tunability (TL-1, TL-2 and TN concepts shown in Fig. 6), we present in the main body of the text closed-form expressions of their elastic properties, and in Appendix E their buckling and yielding strength. For unit cells with transverse isotropic or isotropic CTEs (concepts shown in Fig. 7 and 8), a numeric approach is used to obtain their elastic properties.

\subsection{Relative density}

Tab. 4 lists the relative density $\rho^{*}$, i.e. the volume fraction, of all nine unit cells. $\rho^{*}$ is given by the ratio of the volume of the solid components, $V_{\text {solid }}$, to the volume of the unit cell, $V_{\text {unit cell }}$ : $\rho^{*}=V_{\text {solid }} / V_{\text {unit cell }}$. The expressions rely on the assumption that all high- and low-CTE bars have a circular cross-section with radius $a$, and bars made of identical material have identical length.

\begin{tabular}{|c|c|c|c|}
\hline & TL-1 \& TL-2 & TN & \\
\hline \multirow{2}{*}{$\begin{array}{l}\text { Unidirectional } \\
\text { CTE }\end{array}$} & $4 \pi a^{2}(\cos \theta+1)$ & $6 \pi a^{2}(2 \cos \theta+1)$ & \multirow{2}{*}{$(21)$} \\
\hline & $\overline{l_{1}^{2} \xi}$ & $\overline{l_{1}^{2} \zeta}$ & \\
\hline \multirow{2}{*}{$\begin{array}{c}\text { Transverse } \\
\text { Isotropic CTE }\end{array}$} & $2 \pi a^{2}(3+4 / \cos \theta+\sqrt{3} \cos \gamma)$ & $4 \pi a^{2}(11+6 / \cos \theta+\cos \gamma)$ & \multirow{2}{*}{$(23)$} \\
\hline & $\overline{\sqrt{3} \sin ^{3} \theta l_{1}^{2}\left(\tan ^{-1}(\gamma)+\xi / \cos \theta\right)^{2}}(22)$ & $\sin \gamma l_{1}^{2}(1+\cos \gamma+\sin \gamma \zeta / \cos \theta)^{2}$ & \\
\hline \multirow{2}{*}{ Isotropic CTE } & $6 \sqrt{2} \pi a^{2}(5+8 / \cos \theta)$ & $162 \pi^{2}(1+1 / \cos \theta)$ & \multirow{2}{*}{$(25)$} \\
\hline & $l_{1}^{2}(1+\xi / \cos \theta)^{3}$ & $l_{1}^{2}(\sqrt{2}+\zeta / \cos \theta)^{3}$ & \\
\hline
\end{tabular}

Table 4: Relative density of all nine unit cells, where $l_{1}$ is the length of high-CTE bars with circular cross section of radius $a, \gamma$ is the deviation angle (Fig. 7), $\zeta=\left(3-4 \cos ^{2} \theta\right)^{1 / 2}$, and $\xi=\left(1-2 \cos ^{2} \theta\right)^{1 / 2}$. 


\subsection{Elastic stiffness}

The continuum-based modeling approach offers a practical and efficient method for analyzing a periodic medium (Arabnejad and Pasini, 2013; Vigliotti et al., 2014). It is based on the substitution of the discrete model with an equivalent continuum. A number of approaches, such as those relying on standard mechanics and asymptotic homogenization theory (Hollister and Kikuchi, 1992), have been proposed for developing continuum models, and for determining the appropriate constitutive relations (Vigliotti et al., 2014). Using the standard mechanics approach (Hualin and Wei, 2006) (see Appendix C for more details), here we examine the three unit cells with unidirectional CTE tunability (Fig. 6a to c) for demonstration purposes. The macroscopic stiffness tensor for the TL-1 concept:

$$
\overline{C_{T L-1}}=\frac{\pi a^{2}}{l_{1}^{2} \xi}\left(\begin{array}{cccccc}
\frac{E_{s 1} \cos \theta}{2}+8 E_{s 2} \cos ^{4} \theta+\frac{E_{s 2}}{4} & \frac{E_{s 1} \cos \theta}{2}+\frac{E_{s 2}}{4} & 4 E_{s 2} \cos ^{2} \theta \xi^{2} & 0 & 0 & \frac{E_{s 1} \cos \theta}{2}+\frac{E_{s 2}}{4} \\
\frac{E_{s 1} \cos \theta}{2}+8 E_{s 2} \cos ^{4} \theta+\frac{E_{s 2}}{4} & 4 E_{s 2} \cos ^{2} \theta \xi^{2} & 0 & 0 & \frac{E_{s 1} \cos \theta}{2}+\frac{E_{s 2}}{4} \\
& 4 E_{s 2} \xi^{4} & 0 & 0 & 0 \\
& & 4 E_{s 2} \cos ^{2} \theta \xi^{2} & 0 & 0 \\
& & & 4 E_{s 2} \cos ^{2} \theta \xi^{2} & 0 \\
& & & & & \frac{E_{s 1} \cos \theta}{2}+\frac{E_{s 2}}{4}
\end{array}\right),
$$

where $\overline{C_{T L-1}}$ is the effective stiffness matrix of TL-1 concept and $E_{s 1}, E_{s 2}$ are Young's modulus of two components, respectively. The stiffness matrix in Eq. (26) shows a typical material in an orthorhombic system referring to the coordinate system shown in Appendix C. Analogously, for the TL-2 concept the stiffness tensor is:

$$
\overline{C_{T L-2}}=\frac{\pi a^{2}}{l_{1}^{2} \xi}\left(\begin{array}{cccccc}
E_{s 1} \cos \theta+8 E_{s 2} \cos ^{4} \theta & E_{s 1} \cos \theta & 4 E_{s 2} \cos ^{2} \theta \xi^{2} & 0 & 0 & 0 \\
& E_{s 1} \cos \theta+8 E_{s 2} \cos ^{4} \theta & 4 E_{s 2} \cos ^{2} \theta \xi^{2} & 0 & 0 & 0 \\
& & 4 E_{s 2} \xi^{4} & 0 & 0 & 0 \\
& & & 4 E_{s 2} \cos ^{2} \theta \xi^{2} & 0 & 0 \\
& & & & 4 E_{s 2} \cos ^{2} \theta \xi^{2} & 0 \\
& & & & & E_{s 1} \cos \theta
\end{array}\right),
$$


which shows a typical material in a tetragonal system. The macroscopic stiffness tensor for the TN concept can be written as:

$$
\overline{C_{T N}}=\frac{2 \pi a^{2}}{l_{1}^{2} \zeta}\left(\begin{array}{ccccc}
\frac{9 E_{s 1} \cos \theta}{4}+2 E_{s 2} \cos ^{4} \theta & \frac{3 E_{s 1} \cos \theta}{4}+\frac{2 E_{s 2} \cos ^{4} \theta}{3} & \frac{2}{3} E_{s 2} \cos ^{2} \theta \zeta^{2} & 0 & \frac{2 E_{s 2} \cos ^{3} \theta \zeta}{3} \\
\frac{9 E_{s 1} \cos \theta}{4}+2 E_{s 2} \cos ^{4} \theta & \frac{2}{3} E_{s 2} \cos ^{2} \theta \zeta^{2} & 0 & -\frac{2 E_{s 2} \cos ^{3} \theta \zeta}{3} & 0 \\
& \frac{1}{9} E_{s 2} \zeta^{4} & 0 & 0 & 0 \\
\text { Symm. } & \frac{2}{3} E_{s 2} \cos ^{2} \theta \zeta^{2} & 0 & -\frac{2 E_{s 2} \cos ^{3} \theta \zeta}{3} \\
& & \frac{2}{3} E_{s 2} \cos ^{2} \theta \zeta^{2} & 0 \\
& & & \frac{3 E_{s 1} \cos \theta}{4}+\frac{2 E_{s 2} \cos ^{4} \theta}{3}
\end{array}\right),
$$

which is the stiffness tensor of a typical material in a trigonal system. The homogenized stiffness matrix relates the macroscopic strains to the macroscopic stresses of the homogenized material. Tab. 5 reports the closed form expressions of the effective Young's modulus of the three concepts in the vertical direction (i.e. the principal direction with CTE tunability, $x_{3}$ ) and shear modulus in the $x_{1}-x_{2}$ plane.

\begin{tabular}{|c|c|c|}
\hline $\begin{array}{c}\text { Unidirectional } \\
\text { CTE Concepts }\end{array}$ & $E_{33}$ & $G_{12}$ \\
\hline TL-1 & $\frac{E_{s 1} E_{s 2} \xi^{4}}{\left(E_{s 1}+4 E_{s 1} \cos ^{4} \theta+2 E_{s 2} \cos ^{3} \theta\right)(\cos \theta+1)} \rho^{*}$ & $\frac{E_{s 1} E_{s 2} \cos \theta}{2(\cos \theta+1)\left(E_{s 2}+2 E_{s 1} \cos \theta\right)} \rho^{*}$ \\
\hline TL-2 & $\frac{E_{s 1} E_{s 2} \xi^{4}}{\left(E_{s 1}+4 E_{s 2} \cos ^{3} \theta\right)(\cos \theta+1)} \rho^{*}$ & $\frac{E_{s 1} \cos \theta}{4(\cos \theta+1)} \rho^{*}$ \\
\hline $\mathrm{TN}$ & $\frac{E_{s 1} E_{s 2} \zeta^{4}}{\left(9 E_{s 1}+8 E_{s 2} \cos ^{3} \theta\right)(2 \cos \theta+1)} \rho^{*}$ & $\frac{E_{s 1} \cos \theta}{4(2 \cos \theta+1)} \rho^{*}$ \\
\hline
\end{tabular}

Table 5: Effective Young's modulus (in direction $x_{3}$ ) and shear modulus (on the $x_{1}-x_{2}$ plane) for TL-1, TL2, and TN concepts with unidirectional CTE tunability.

Similar to the CTE maps presented in section 2.2.2 for the three building blocks, TL-1, TL-2 and TN, Fig. 12 visualizes the spatial variation of the Young's moduli in spherical coordinates for 
their corresponding unidirectional concepts. The symmetries of the surfaces shown in Fig. 12 also feature the geometrical symmetry of each concept: Fig. 12a for the orthorhombic system, Fig. $12 \mathrm{~b}$ for the tetragonal system, and Fig. $12 \mathrm{c}$ for the trigonal system.
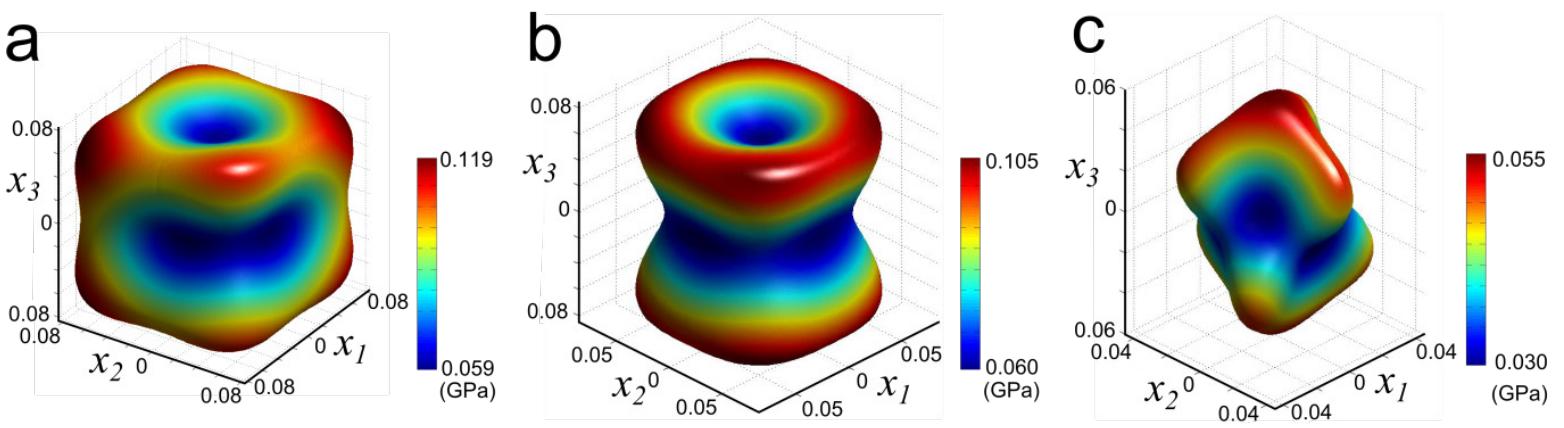

Figure 12: Three-dimensional surfaces showing the variation of Young's moduli along the principal directions for (a) TL-1, (b) TL-2 and (c) TN concepts. The length of the radius vector is the Young's moduli of each concept taken $\mathrm{Al} / \mathrm{Ti}$ as components. All concepts have identical beam length to diameter ratio of 0.04 .

As shown in Fig. 13, for concepts with unidirectional CTE tunability, the normalized specific Young's moduli and shear moduli change with the skew angle and Young's modulus ratio of components (i.e. Young's moduli of low-CTE material over high-CTE material: $\left.E_{s 2} / E_{s 1}\right)$. In Fig. 13, an increase in skew angle causes rising Young's modulus and falling shear modulus due to concept degeneration. For all the three concepts, the normalized vertical Young's moduli gradually converge to 1 and the normalized shear moduli gradually converge to 0 . Fig. $13 \mathrm{a}$ and $\mathrm{b}$ also indicate that, for each concept, the effective stiffness, including both Young's and shear moduli, increases with the stiffness ratio of the components. This is caused by the low-CTE material (material two in this work) predominantly located along the $x_{3}$ direction with stiffness $\left(E_{s 2}\right)$ increasing with the component stiffness ratio. Comparing concepts of given skew angle, the TN concept generally has Young's moduli in the vertical direction higher than those of stationary lines. In contrast, concepts constructed via TL-1 and TL-2 have similar vertical Young's moduli. As shown in Fig. 13b, no concept is absolutely superior to others for all skew 
angles, as the shear moduli converge at different rates to zero with $\theta$ approaching $90^{\circ}$. In addition to stiffness, buckling and yielding strength, other properties that pertain to structural efficiency, are derived and summarized in Appendix D.
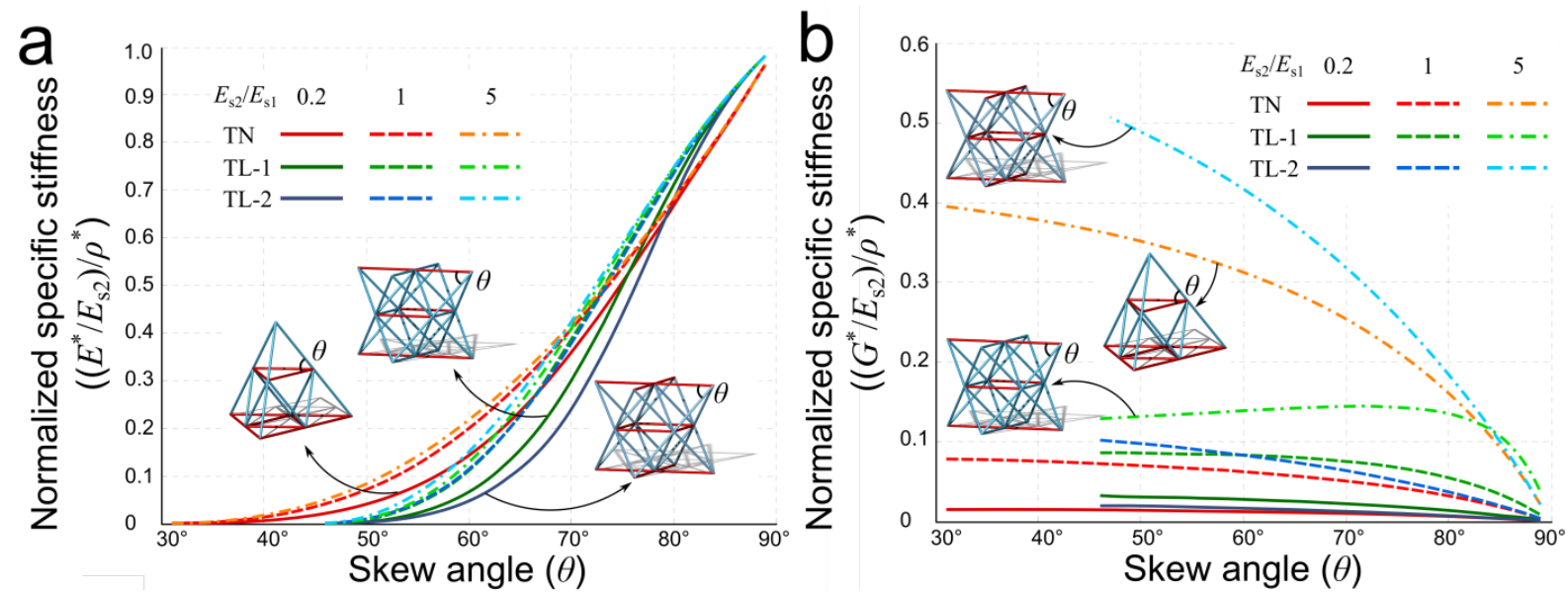

Figure 13: Normalized specific stiffness in the vertical direction for TL-1, TL-2, and TN building blocks as a function of the skew angle for selected values of the stiffness ratio of the components $\left(E_{s 2} / E_{s 1}\right)$ : (a) Young's modulus and (b) shear modulus. $\rho^{*}$ represents relative density.

\section{Discussion on thermal expansion, specific stiffness, and their}

\section{trade-off}

Thermal expansion. The CTE of bi-material lattice materials depends on the CTE mismatch of materials, the skew angle, i.e. the interplay of the structural members, as well as the stiffness mismatch of materials and joints. Fig. 14 reveals the role of the skew angle in the thermal expansion performance of each low-CTE unit cell. As a general trend, we observe that as the skew angle increases from the minimum to the maximum value of $\theta$ range (Tab. 2), the CTEs for all units converge gradually to that of the low-CTE solid material $\left(10 \times 10^{-6} /{ }^{\circ} \mathrm{C}\right.$ in Fig. 14). The similar upper bound indicates the largest $\triangle C T E$ comes from the concept obtaining the lowest CTE value. As shown in Fig. 14, with a given range of skew angles and given directional behaviour (i.e. unidirectional, transverse isotropic, or isotropic), the lowest effective CTE a TL-1 
and TL-2 concept can achieve is generally lower than that of the TN concept. This demonstrates that concepts with stationary lines have a better CTE tunability, because the CTE tunability of TL-1 and TL-2 building blocks is higher than that of the TNs (Fig. 4). Similarly, when comparing TL-1 with TL-2 concepts, the latter has generally a larger $\triangle C T E$, especially for concepts with small or no monomaterial core (i.e. transverse-isotropic and unidirectional, respectively). However, for isotropic concepts, above $\theta=53^{\circ}$ TL-2 has higher CTE values than TL-1, as the influence of TL-2 high-CTE octahedron core is far larger than that of the low-CTE core of the TL-1 concept. For lattices assembled with a given building block, TL-1, TL-2, or TN, the CTE tunability is often the smallest for unit cells with isotropic CTE, followed by unit cells with transverse isotropic CTE. This can also be attributed to the influence of larger single-material core (octahedron) of isotropic-concepts than the core (triangular bipyramid) of the transverseisotropic concepts; the former is more effective in counteracting the CTE tunability via larger monomaterial core and hence impairing CTE tunability. On the other hand, the largest CTE tunability in the principal direction can be achieved by unit cells which have unidirectional CTE with no monomaterial core.

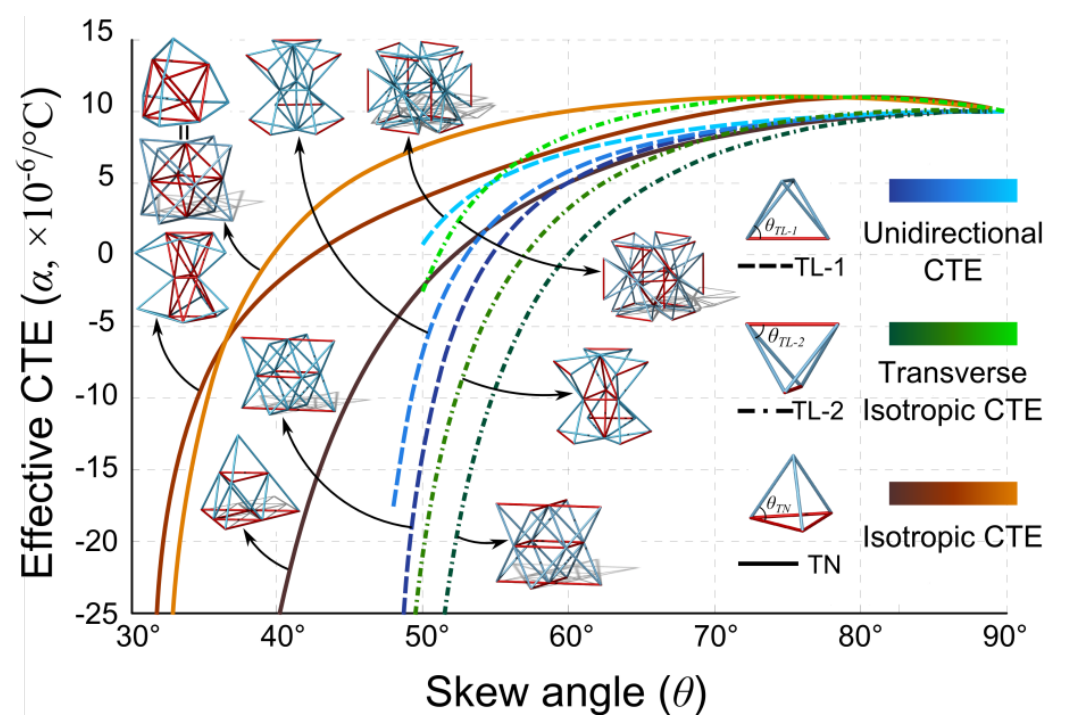

Figure 14: Relation between CTEs and skew angle for the nine unit cells here under investigation. A high- 
CTE material of $\alpha_{s 1}=20 \times 10^{-6} /{ }^{\circ} \mathrm{C}$ and a low-CTE material of $\alpha_{s 2}=10 \times 10^{-6} /{ }^{\circ} \mathrm{C}$ are taken here as representative components. For unit cells with unidirectional low CTE, the CTE refers to the vertical direction $\left(x_{3}\right)$. For unit cells with transverse isotropic and isotropic low CTE, the effective CTE refers to the principal directions in the plane $x_{1}-x_{2}$. Each line style indicates one of the three building blocks which a unit cell is built from. The CTE of a benchmark isotropic concept (Steeves et al., 2007) with identical effective CTE to the isotropic TN concept is also shown in purple for comparison.

The experimental results in section 3.4 show that the CTEs under the pin- and rigid-jointed assumptions deviate marginally from each other. Under the pin-jointed assumption, the stiffness mismatch of the component materials has no effect on the effective CTE, as opposed to the case of the rigid-jointed assumption, where the stiffness mismatch of the components can play a role on CTE tunability. A relative comparison between the three factors here examined shows that the effect of the stiffness mismatch is secondary to the influence of the other two, namely the interplay of structures and the CTE mismatch of materials. Appendix A reports additional results on the CTE of rigid-jointed bi-material lattices obtained for a number of Young's modulus ratio of the constituent solids.

Specific stiffness. As per the sensitivity of the elastic properties, Fig. 15 shows computational results of their normalized Young's modulus in the direction of CTE tunability, versus both $\rho^{*}$, relative density, and $\theta$, skew angle. The relative density ranges from 0 to 0.2 . Different representative fields of the skew angle are selected for each concept to indicate the variation trend of unit cell stiffness. Along the vertical axes, sketches show the variation of the unit cell geometry with the skew angle.

The specific stiffness of all concepts increases linearly with $\rho^{*}$. For increasing $\theta$ with constant $\rho^{*}$, the Young's modulus is also observed to raise for unidirectional concepts (Fig. 15a to c), while a parabolic effect appears for the remaining concepts (e.g. Fig. $15 f$ and i), i.e. a rise is 
followed by declining modulus. This can be attributed to the changing alignment of the highstiffness component (i.e. low-CTE Ti bars) along the loading direction: i) for unidirectional concepts, increasing $\theta$ results in an incremental alignment along $x_{3}$; ii) for both transverseisotropic and isotropic concepts, the same incremental alignment is initially experienced along the loading direction, before beginning to deviate from the loading direction above a specific $\theta$ value. The parabolic effect can also be observed for Fig. 15d, e, g, h, and j when a larger range of $\theta$ is plotted.

For given $\rho^{*}$ and $\theta$, Fig. 15 highlights also similarities between the stiffness of TL-1 and TL-2 concepts. Compared to TL-2 concepts, TL-1 concepts feature slightly higher values of specific stiffness, due to presence of more Ti bars. However, TN concepts generally outperform the others in stiffness, especially for transverse-isotropic (Fig. 15f) and isotropic concepts (Fig. 15i) where the parabolic effect is observed at $\theta \approx 60^{\circ}$, resulting in a much higher specific stiffness than TL concepts with identical $\theta, \rho^{*}$, and CTE directionality. Fig. $15 \mathrm{j}$ plots also the specific stiffness values for the benchmark (Steeves et al., 2007). Compared to this baseline, we notice that unidirectional and transverse-isotropic concepts produce higher stiffness along the direction of CTE tunability, but the isotropic concepts are comparable for given geometric and constituent parameters. 
a

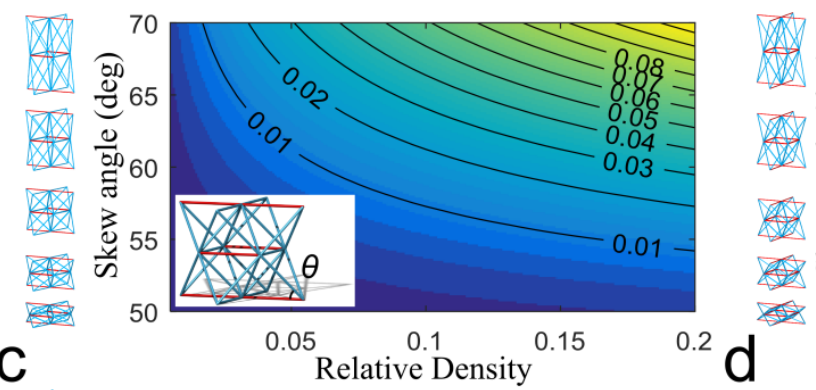

C

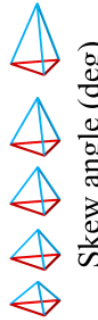

e

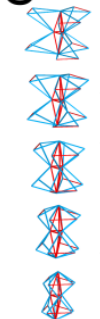

g
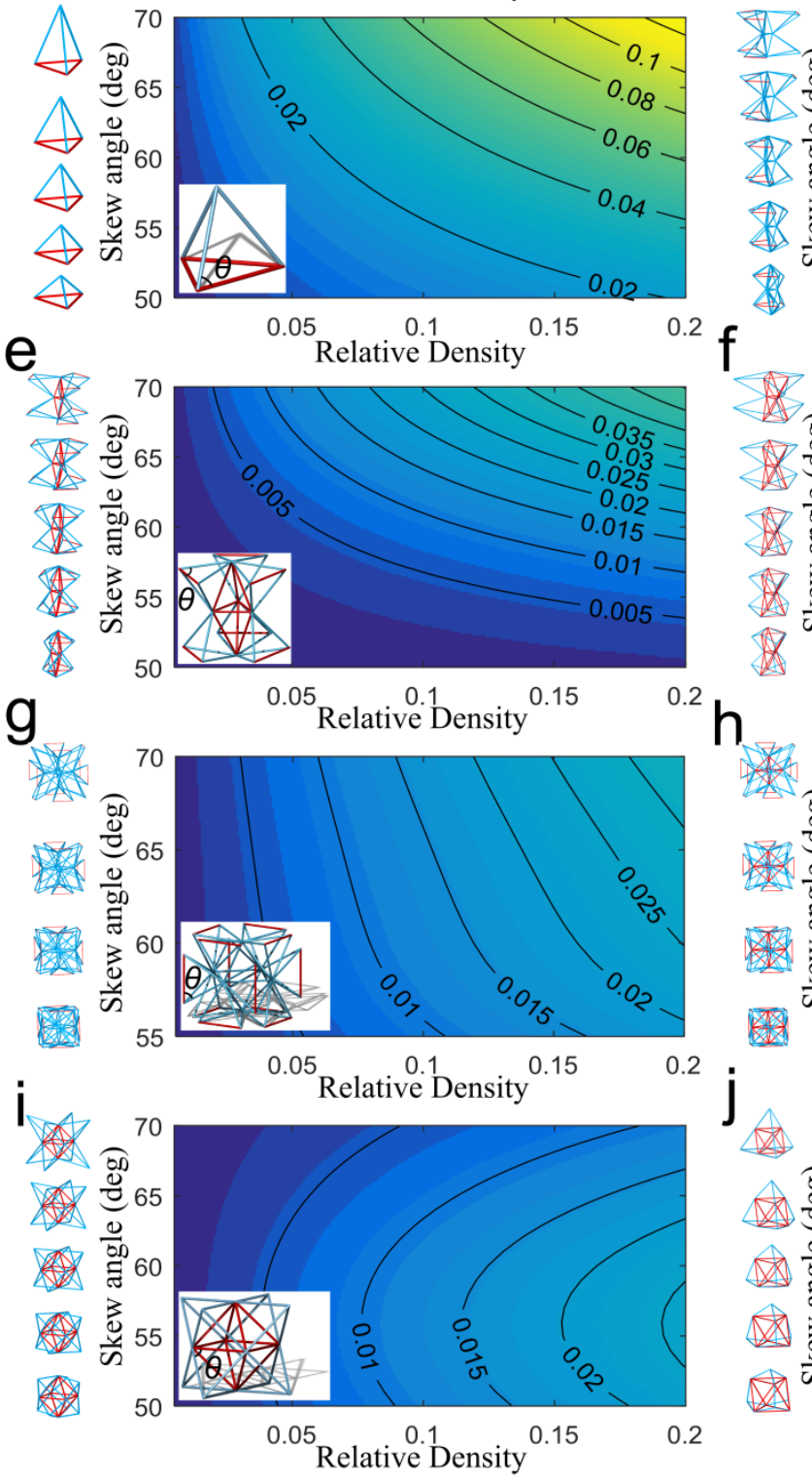
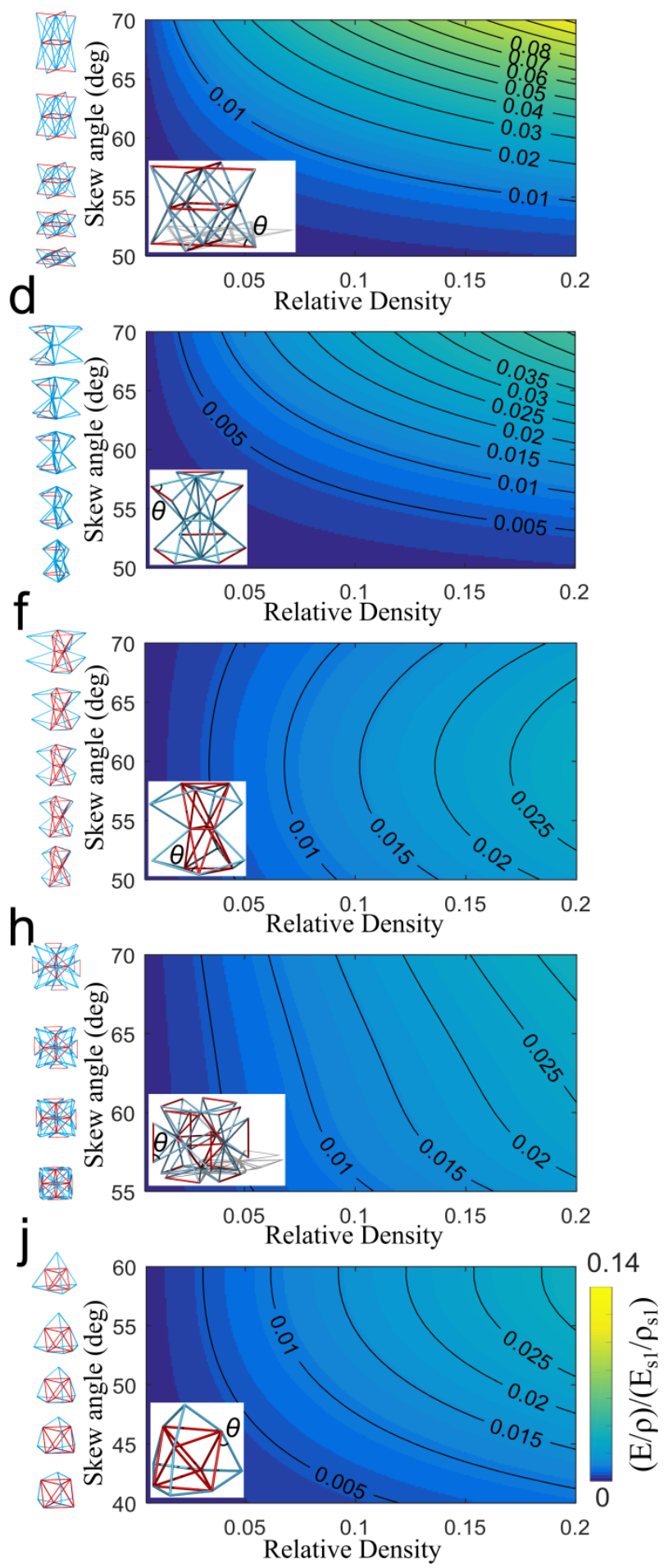

Figure 15: Contour plots for specific stiffness $\left((E / \rho) /\left(E_{s 1} / \rho_{s 1}\right)\right.$ with $E$ representing the effective stiffness in the CTE tunable direction and $\rho$ representing the density of the lattice) versus skew angle and relative density: Young's modulus in the vertical direction of cell topologies with unidirectional CTE: (a) TL-1, (b) TL-2, and (c) TN concept; Young's modulus in the horizontal direction of cell topologies with transverse 
isotropic CTE: (d) TL-1, (e) TL-2, and (f) TN concept; and Young's modulus of cell topologies with isotropic CTE: (g) TL-1, (h) TL-2, and (i) TN concept. S-concept (Steeves et al., 2007) is plotted as a benchmark in (j) to compare elastic performances.

Trade-off between CTE tunability and specific stiffness. The results above give an indication of existing trade-offs between the properties of the concepts here studied: $\triangle C T E$ and specific stiffness. To better understand these trade-offs, Fig. 16a shows bars of $\triangle C T E$ and specific stiffness simultaneously, and in Fig. 16b $\triangle C T E$ is plotted versus specific stiffness to demonstrate Pareto-fronts, both for all concepts made of $\mathrm{Al}$ and $\mathrm{Ti}$.

For Fig. 16a, the $\triangle C T E$ bars are calculated for all concepts under a given stiffness value (1 GPa), while bars of structural efficiency are all derived from concepts with equal CTE $\left(6.5 \times 10^{-6} /{ }^{\circ} \mathrm{C}\right)$, a value lower than the CTE of their base materials: $\mathrm{Al} / \mathrm{Ti}$ ). This allows for a consistent comparison of their thermo-elastic performance. The bars are arranged in order of increasing number of CTE tunable principal directions, from unidirectional to isotropic concepts. Evidently, with increasing number of CTE tunable directions, there is a penalty in both $\triangle C T E$ and structural efficiency. The decrease in $\triangle C T E$ is due to the presence and increasing size of monomaterial cores, as previously observed. The drop in structural efficiency, however, is attributed to the distribution of load bearing bars along the CTE tunable directions, thus resulting in a more uniform load capacity, but with lower effective stiffness along a specific direction. For unidirectional and transverse-isotropic concepts, TL-2 unit cells provide the best $\triangle C T E$ and specific stiffness values, followed closely by TN concepts, while both performing significantly better than TL-1. However, for isotropic concepts, the TN thermo-elastic performance exceeds that of TL-2, due to the reduced performance of the TL-2 core. 

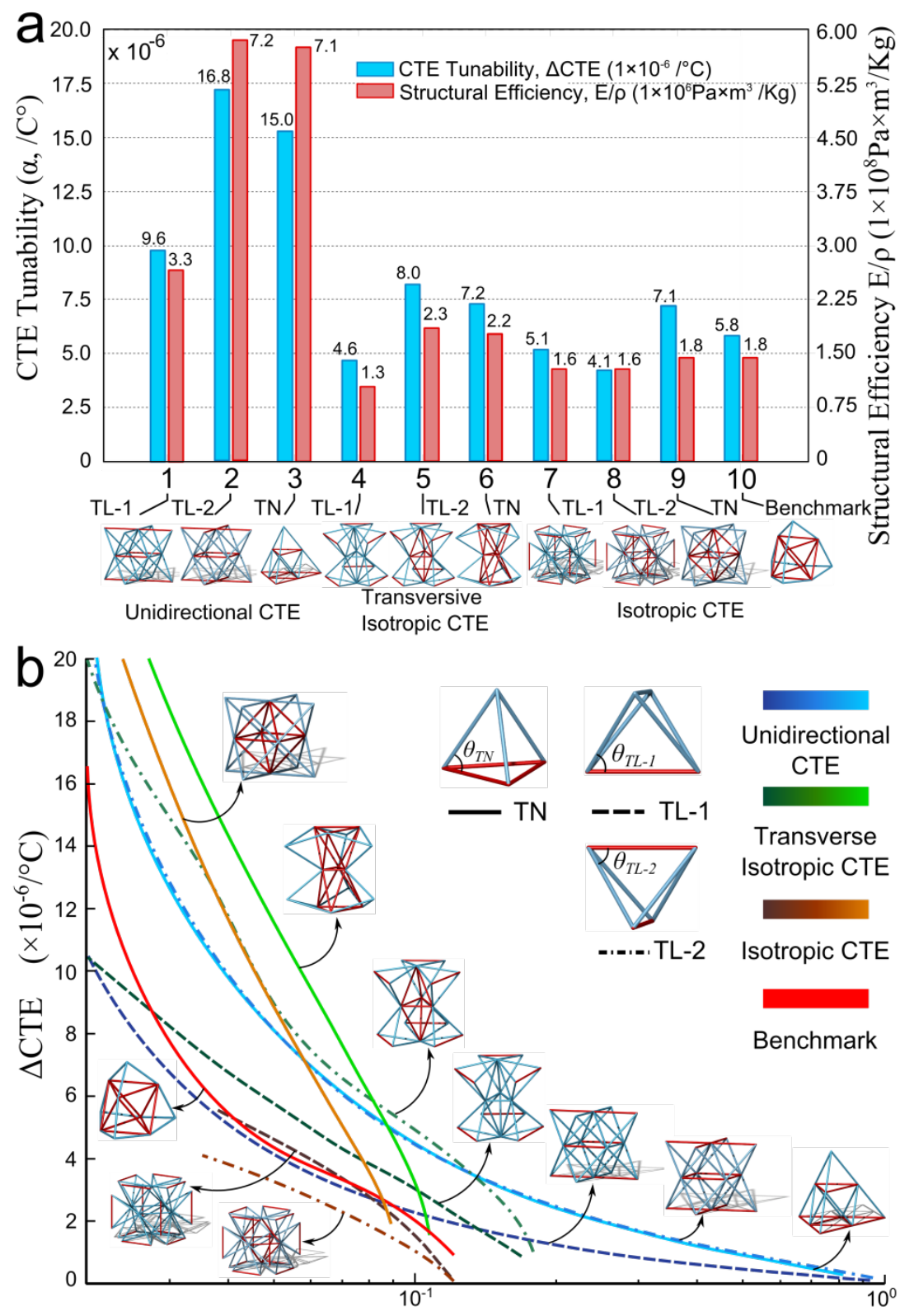

$(\mathrm{E} / \rho) /\left(\mathrm{E}_{\mathrm{s} 1} / \rho_{\mathrm{s} 1}\right)$

Figure 16: (a) Comparison of proposed and existing bi-material concepts for given bar thickness ratio of 0.04 on the basis of (i) CTE tunability ( $\triangle C T E=\operatorname{Max} C T E-\operatorname{Min} C T E$ ) shown as blues bars for Young's modulus of 1GPa in the CTE tunable direction, and (ii) specific stiffness (Young's modulus/Density: $E / \rho$ ) for prescribed CTE of $6.5 \times 10^{-6} /{ }^{\circ} \mathrm{C}$, in red. (b) CTE tunability plotted versus structural efficiency. The relative specific stiffness and CTE tunability are plotted in the vertical direction for unidirectional and isotropic CTE concepts, and in the horizontal direction for other concepts. The thermoelastic properties of a benchmark isotropic concept (Steeves et al., 2007) is also shown in purple for comparison.

The Pareto-front curves shown in Fig. 16b are generated from a parametric study of the unit cells, where the skewness angle and the thickness-to-length ratio are the active variables for given 
materials (Al/Ti). The curve shapes emphasize the trade-offs between the two metrics plotted: an attempt of increasing structural efficiency results in a reduced $\triangle C T E$. This common trend demonstrates that the desired deformation that a large CTE tunability would require is generally antagonist to the high specific stiffness that is distinctive of a structurally efficient architecture. Here we notice that unidirectional concepts excel in structural efficiency along their CTE tunable direction; but for increasing $\triangle C T E$, their specific stiffness drops. Transverse-isotropic unit cells are unable to attain the highest level of specific stiffness, but, within their attainable range, they generally provide higher $\triangle C T E$ compared to unidirectional concepts. Finally, for isotropic concepts, including S-concept (Steeves et al., 2007), there is a sacrifice in both $\triangle C T E$ and structural efficiency, except for the isotropic TN unit cell, which maintains performance similar to its transverse-isotropic counterpart. Overall, Fig. 16a and b provides a comprehensive comparison of the thermo-elastic performance of the concepts here examined, bearing in mind that no CTE directionality is ultimately superior to the rest as the direction of CTE tunability is entirely dependent on the application requirements. Other structural properties, such as yield and buckling strength, have been evaluated for specific concepts, as examples, and their expressions are provided in Appendix D.

In summary, this work has added to the body of the existing literature three fundamental aspects. The first is on the concepts and their mechanisms of thermal deformation. Three groups (lowCTE, intermediate-CTE, high-CTE) are introduced for the tetrahedral building block to tune the effective CTE at values that can be lower, in between, or higher than the constituent material CTEs. For the low-CTE group, three specific mechanisms are identified for a bi-material tetrahedron: two with stationary-lines, and the third with a stationary-node, the only one known in the literature. The second novel feature of this work is a systematic method to rationally assemble building blocks into compound units, such as the nine here introduced, that can attain 
desired magnitude and directionality of thermal expansion in three-dimensional lattices. The third is a path forward for resolving the trade-off between CTE tunability and specific stiffness, which has led to the assembly of building blocks into stiff and strong, yet high CTE tunable spatial lattices with application across the spectrum of length scale.

\section{Conclusions}

This paper has investigated thermal expansion of spatial bi-material lattices built from tetrahedral building blocks. The material length vector and thermal displacement vector are first defined to assess thermal expansion of truss concepts, and then used to examine all the possible material permutations that can occur in the struts of a dual-material tetrahedron. Drawing from concepts of crystallography and tessellation, a scheme has been presented to understand the relationships between CTE tunability of tetrahedral building blocks, structural symmetry of unit cells, independent elements in the CTE tensor, and effective thermoelastic properties of periodic truss, which can be built from single units as well as more complex assembly of compound units. The results establish underlying principles governing tailorable thermal expansion in dual-material lattice materials, where desired CTE magnitude and spatial CTE directionality can be programmed a priori to satisfy given CTE requirements, such as unidirectional, transverse isotropic, or isotropic. Closed-form expressions for predicting and tuning CTE performance are validated through computational and experimental studies on proof-of-concept prototypes. Results illustrated in maps have been discussed within the context of structural efficiency and CTE tunability, two properties that are generally in conflict at a degree dependent on the unit cell type. This works has also shown that three-dimensional lattices can be systematically assembled with high specific stiffness to attain large CTE tunability over a substantial range of temperature, thus appealing to a large palette of applications where low mass, thermal stability and thermal actuation are primary goals. 


\section{Funding}

This work was supported by the Natural Sciences and Engineering Research Council of Canada [grant number 208241].

\section{Acknowledgments}

The authors gratefully acknowledge the technical support of Mr. Daniel Fraschetti and Mr. Joseph $\mathrm{Lu}$ who assisted in conducting the experiments. $\mathrm{H}$. Xu acknowledges the financial support provided by the China Scholarship Council [File No. 201306020030].

Declarations of interest: none.

\section{References}

Ai, L., Gao, X.L., 2017. Metamaterials with negative Poisson's ratio and non-positive thermal expansion. Compos. Struct. 162, 70-84.

Ai, L., Gao, X.L., 2018. Three-dimensional metamaterials with a negative Poisson's ratio and a non-positive coefficient of thermal expansion. Int. J. Mech. Sci. 135, 101-113.

Arabnejad, S., Pasini, D., 2013. Mechanical properties of lattice materials via asymptotic homogenization and comparison with alternative homogenization methods. Int. J. Mech. Sci. 77, 249-262.

Ashby, M.F., 2011. Materials Selection in Mechanical Design, Fourth ed. ButterworthHeinemann, Oxford.

Boatti, E., Vasios, N., Bertoldi, K., 2017. Origami metamaterials for tunable thermal expansion. Adv. Mater., 1700360.

Breger, J.C., Yoon, C., Xiao, R., Kwag, H.R., Wang, M.O., Fisher, J.P., Nguyen, T.D., Gracias, D.H., 2015. Self-Folding Thermo-Magnetically Responsive Soft Microgrippers. ACS Appl. Mater. Interfaces 7, 3398-3405.

Ding, Z., Yuan, C., Peng, X., Wang, T., Qi, H.J., Dunn, M.L., 2017. Direct 4D printing via active composite materials. Sci. Adv. 3, e1602890.

Fan, H.L., Fang, D.N., Jing, F.N., 2008. Yield surfaces and micro-failure mechanism of block lattice truss materials. Mater. Des. 29, 2038-2042.

Ge, Q., Sakhaei, A.H., Lee, H., Dunn, C.K., Fang, N.X., Dunn, M.L., 2016. Multimaterial 4D Printing with Tailorable Shape Memory Polymers. Sci. Rep. 6, 31110.

Gilmore, D.G., 2002. Spacecraft Thermal Control Handbook: Fundamental technologies. Aerospace Press, United States.

Grima, J.N., Attard, D., Gatt, R., 2008. Truss - type systems exhibiting negative compressibility. Phys. Status Solidi B 245, 2405-2414. 
Hollister, S.J., Kikuchi, N., 1992. A comparison of homogenization and standard mechanics analyses for periodic porous composites. Comput. Mech. 10, 73-95.

Hopkins, J.B., Lange, K.J., Spadaccini, C.M., 2013. Designing microstructural architectures with thermally actuated properties using freedom, actuation, and constraint topologies. Journal of Mechanical Design, Trans. ASME 135, 061004.

Hualin, F., Wei, Y., 2006. An equivalent continuum method of lattice structures. Acta Mech. Solida Sin. 19, 103-113.

Ito, T., Suganuma, T., Wakashima, K., 2000. A micromechanics-based analysis for tailoring glass-fiber-reinforced thermoplastic laminates with near-zero coefficients of thermal expansion. Compos. Sci. Technol. 60, 1851-1861.

Jefferson, G., Parthasarathy, T.A., Kerans, R.J., 2009. Tailorable thermal expansion hybrid structures. Int. J. Solids Struct. 46, 2372-2387.

Kim, T., Hodson, H.P., Lu, T.J., 2004. Fluid-flow and endwall heat-transfer characteristics of an ultralight lattice-frame material. Int. J. Heat Mass Transfer 47, 1129-1140.

Korthuis, V., Khosrovani, N., Sleight, A.W., Roberts, N., Dupree, R., Warren, W.W., Jr., 1995. Negative Thermal Expansion and Phase Transitions in the ZrV2-xPxO7 Series. Chem. Mater. 7, 412-417.

Lakes, R., 2007. Cellular solids with tunable positive or negative thermal expansion of unbounded magnitude. Appl. Phys. Lett. 90.

Lehman, J., Lakes, R., 2013. Stiff, strong zero thermal expansion lattices via the Poisson effect. J. Mater. Res. 28, 2499-2508.

Lehman, J., Lakes, R.S., 2014. Stiff, strong, zero thermal expansion lattices via material hierarchy. Compos. Struct. 107, 654-663.

Lim, T.-C., 2005. Anisotropic and negative thermal expansion behavior in a cellular microstructure. J. Mater. Sci. 40, 3275-3277.

Lim, T.-C., 2013. Negative thermal expansion in transversely isotropic space frame trusses. Phys. Status Solidi B 250, 2062-2069.

Liot, F.c., 2009. Thermal Expansion and Local Environment Effects in Ferromagnetic Iron-Based Alloys-A Theoretical Study, Department of Physics, Chemistry and Biology. Linoping University, Sweden, p. 61.

Mao, Y., Ding, Z., Yuan, C., Ai, S., Isakov, M., Wu, J., Wang, T., Dunn, M.L., Qi, H.J., 2016. 3D Printed Reversible Shape Changing Components with Stimuli Responsive Materials. Sci. Rep. 6, 24761.

Mary, T.A., Evans, J.S.O., Vogt, T., Sleight, A.W., 1996. Negative Thermal Expansion from 0.3 to 1050 Kelvin in ZrW2O8. Sci. 272, 90-92.

Miller, W., Mackenzie, D.S., Smith, C.W., Evans, K.E., 2008. A generalised scale-independent mechanism for tailoring of thermal expansivity: Positive and negative. Mech. Mater. 40, 351-361.

Miller, W., Smith, C., Mackenzie, D., Evans, K., 2009. Negative thermal expansion: a review. J. Mater. Sci. 44, 5441-5451.

Mohr, D., 2005. Mechanism-based multi-surface plasticity model for ideal truss lattice materials. Int. J. Solids Struct. 42, 3235-3260.

Nye, J.F., 1985. Physical Properties of Crystals: Their Representation by Tensors and Matrices. Clarendon Press, Oxford, UK.

Palumbo, N., Smith, C., Miller, W., Evans, K., 2011. Near-zero thermal expansivity 2-D lattice structures: Performance in terms of mass and mechanical properties. Acta Mater. 59, 2392-2403. 
Palumbo, N.M.A., 2013. Trusses with Reduced Thermal Expansion: their Design, and Mass and Stiffness Penalties. University of Exeter, UK, p. 260.

Qu, J., Kadic, M., Naber, A., Wegener, M., 2017. Micro-Structured Two-Component 3D Metamaterials with Negative Thermal-Expansion Coefficient from Positive Constituents. Sci. Rep. 7, 40643.

Sigmund, O., Torquato, S., 1997. Design of materials with extreme thermal expansion using a three-phase topology optimization method. J. Mech. Phys. Solids 45, 1037-1067.

Sleight, A.W., 1998. Negative thermal expansion materials. Curr. Opin. Solid State Mater. Sci. 3, 128-131.

Steeves, C.A., dos Santos e Lucato, S.L., He, M., Antinucci, E., Hutchinson, J.W., Evans, A.G., 2007. Concepts for structurally robust materials that combine low thermal expansion with high stiffness. J. Mech. Phys. Solids 55, 1803-1822.

Stoychev, G., Puretskiy, N., Ionov, L., 2011. Self-folding all-polymer thermoresponsive microcapsules. Soft Matter 7, 3277-3279.

Takezawa, A., Kobashi, M., 2017. Design methodology for porous composites with tunable thermal expansion produced by multi-material topology optimization and additive manufacturing. Composites, Part B 131, 21 - 29.

Tibbits, S., 2012. Design to Self-Assembly. Archit. Des. 82, 68-73.

USDOD, 1998. Military Handbook - MIL-HDBK-5H: Metallic Materials and Elements for Aerospace Vehicle Structures. U.S. Department of Defense, U.S.

Vandeperre, L.J., Clegg, W.J., 2003. Tailoring strains through microstructural design. MRS Online Proc. Libr. 785, D11.14.11.

Vigliotti, A., Deshpande, V.S., Pasini, D., 2014. Non linear constitutive models for lattice materials. J. Mech. Phys. Solids 64, 44-60.

Wadley, H.N.G., 2006. Multifunctional periodic cellular metals. Philos. Trans. R. Soc., A 364, 31-68.

Wang, Q., Jackson, J.A., Ge, Q., Hopkins, J.B., Spadaccini, C.M., Fang, N.X., 2016. Lightweight Mechanical Metamaterials with Tunable Negative Thermal Expansion. Phys. Rev. Lett. $117,175901$.

Watts, S., Tortorelli, D.A., 2017. Optimality of thermal expansion bounds in three dimensions. Extreme Mech. Lett. 12, 97-100.

Wei, K., Chen, H., Pei, Y., Fang, D., 2016. Planar lattices with tailorable coefficient of thermal expansion and high stiffness based on dual-material triangle unit. J. Mech. Phys. Solids 86, 173-191.

Wu, L., Li, B., Zhou, J., 2016. Isotropic Negative Thermal Expansion Metamaterials. ACS Appl. Mater. Interfaces 8, 17721-17727.

Xu, H., Farag, A., Pasini, D., 2017. Multilevel hierarchy in bi-material lattices with high specific stiffness and unbounded thermal expansion. Acta Mater. 134, 155-166.

Xu, H., Pasini, D., 2016. Structurally Efficient Three-dimensional Metamaterials with Controllable Thermal Expansion. Sci. Rep. 6, 34924.

Yamamoto, N., Gdoutos, E., Toda, R., White, V., Manohara, H., Daraio, C., 2014. Thin Films with Ultra-low Thermal Expansion. Adv. Mater. 26, 3076-3080.

Zhang, Q., Wommer, J., O’Rourke, C., Teitelman, J., Tang, Y., Robison, J., Lin, G., Yin, J., 2017. Origami and kirigami inspired self-folding for programming three-dimensional shape shifting of polymer sheets with light. Extreme Mech. Lett. 11, 111-120. 


\section{Appendix A: Additional concepts and stiffness mismatch of}

\section{materials}

Fig. A1 depicts two dual-material tetrahedra with CTE tunability that can be used to generate six supplementary unit cells with given CTE behaviour. The first tetrahedron (Fig. A1a) has lowCTE tunability in the vertical direction with shear thermal deformation during expansion. The second tetrahedron, intermediate tetrahedron, is able to obtain the effective CTE of a value between the CTEs of the two components only. The additional concepts shown in Fig. A1 can not only obtain transverse isotropic and isotropic CTE, as with concepts shown in Fig. 7 and Fig. 8, but also orthotropic CTE, as shown in Fig. A1b. The unit cell shown in Fig. A1b has CTE tunability in both the $x_{1}$ and $x_{3}$ directions with different magnitudes. With the $x_{2}$ direction exhibiting no CTE tunability, the concept overall demonstrates orthotropic CTE tunability.
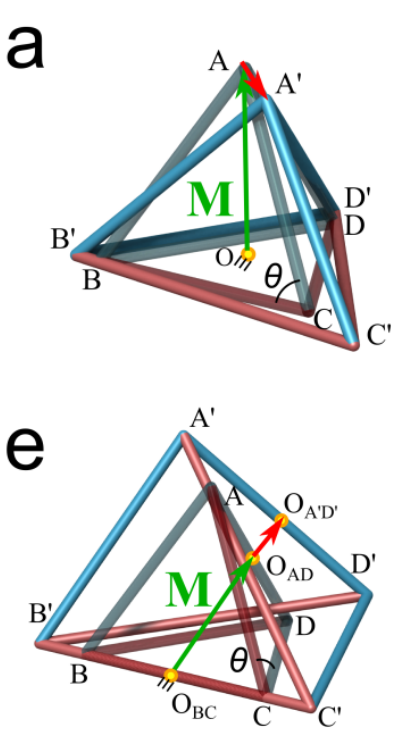

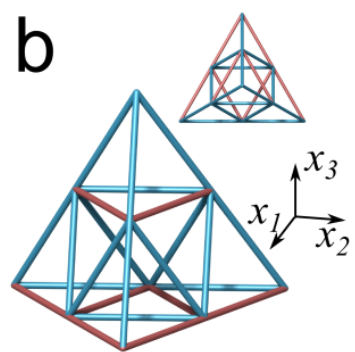

Orthotropic

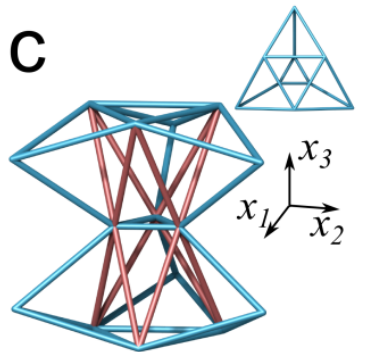

Transverse Isotropic Transverse Isotropic

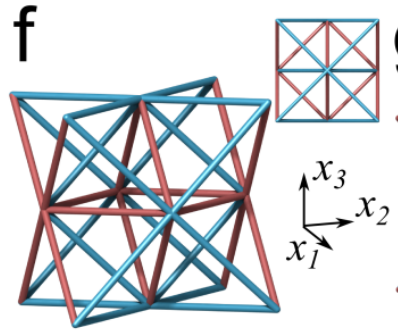

Transverse Isotropic Transverse Isotropic
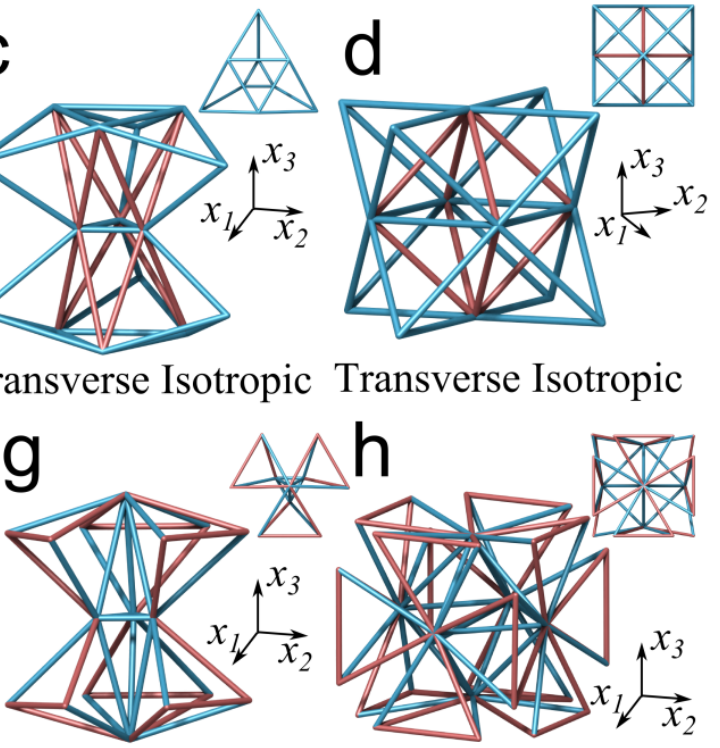

Isotropic

Figure A1: Dual-material tetrahedra along with compound unit cells (a): tetrahedron with low-CTE (four low-CTE bars and two high-CTE bars, L4H2); (b) to (d): unit cells constructed from the low-CTE tetrahedron shown in (a); (e): tetrahedron with intermediate CTE (L3H3); and (f) to (h): unit cells assembled from the low-CTE tetrahedron shown in (e).

The effective CTE of the dual-material tetrahedra shown in Fig. Ala and e (M direction) can be 
expressed respectively as:

$$
\begin{aligned}
& \alpha_{z-\mathrm{L} 4 \mathrm{H} 2}=\frac{16 \alpha_{s 1} \cos ^{2} \theta-\alpha_{s 2}}{16 \cos ^{2} \theta-1}, \theta \in\left(15^{\circ}, 75^{\circ}\right), \\
& \alpha_{z-\mathrm{L} 3 \mathrm{H} 3}=\frac{4 \alpha_{s 1} \cos ^{2} \theta+\alpha_{s 2}}{4 \cos ^{2} \theta+1}, \theta \in\left(36^{\circ}, 72^{\circ}\right) .
\end{aligned}
$$

Fig. A2 shows unit cells with transverse isotropic CTE governed by the deviation angle, $\gamma$, and the skew angle, $\theta$. The decrease of $\gamma$ and increase of $\theta$ allow the connection of unit cells (Fig. A2a to d) in the out-of-plane principal direction via only stationary lines or stationary nodes. In contrast with concepts shown in Fig. 7, which have no CTE tunability in the vertical direction, concepts in Fig. A2 have CTE tunability in all three principal directions. Comparison of concepts depicted in Fig. A2c and d shows the effects of $\gamma$ and $\theta$ on the packing factor, $\varphi$. Differences in the packing factor are controlled by the need of the inner octahedron to thermally expand without touching adjacent cells (Fig. A2k and 1). A packing factor of 50\% could be obtained for tessellation in Fig. A2k. In contrast, dissimilar skew angles for the concept in Fig. A2d results in a tessellation with higher packing factors, i.e. $100 \%$.
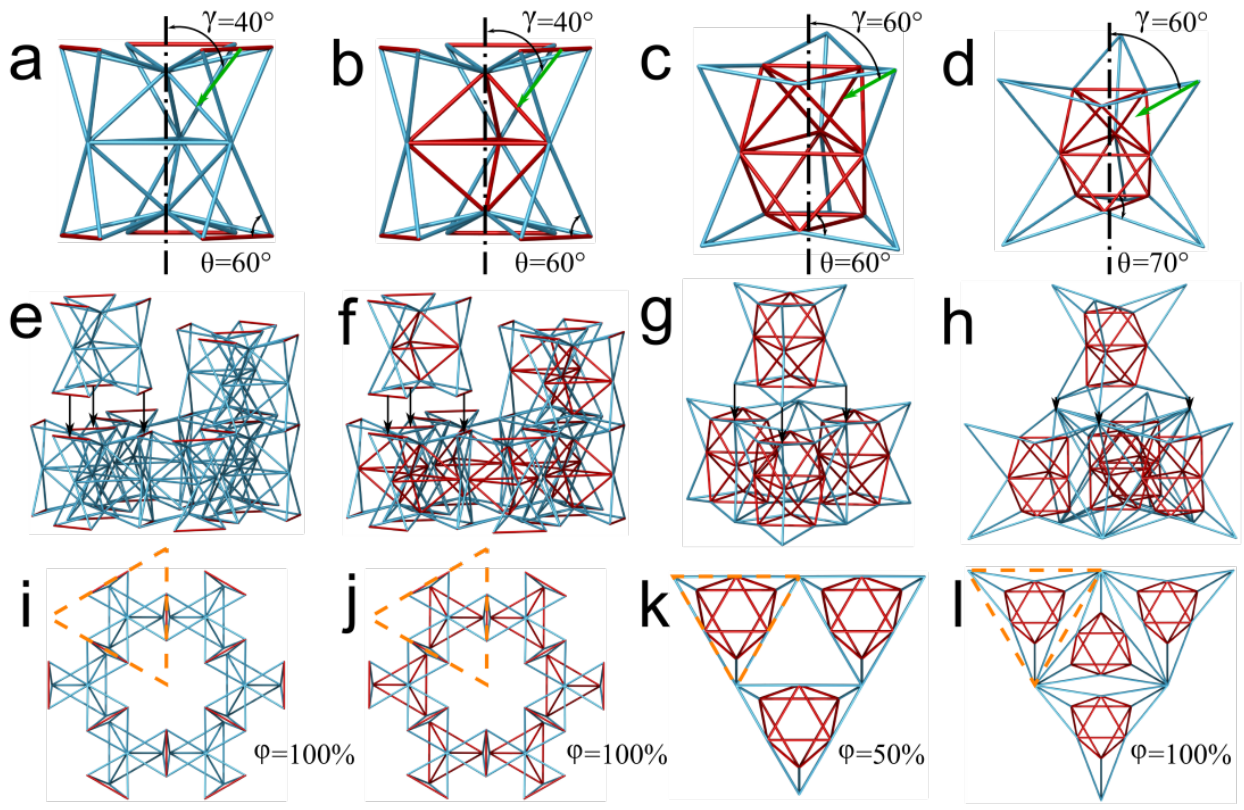
Figure A2: Low-CTE unit cells with transverse isotropic CTE tunability: (a) TL-1 concept, (b) TL-2 concept, (c) and (d) TN concepts. (e) to (h) show an axonometric view of the unit cell assembly; (i) to (l) are the top view of assembly. $\theta, \gamma$, and $\varphi$ are skew angle, deviation angle, and packing factor respectively. Dash yellow line indicates the compound unit cell within the assembly.

In the following we assess the effect of the stiffness mismatch of materials and joint assumption.

Fig. A3 visualizes the effective CTE in the vertical direction for rigid-jointed TL-2 and TN concepts with unidirectional CTE tunability, here chosen for demonstrative purposes. Both the skew angle and the Young's modulus ratio $E_{s 1} / E_{s 2}$ of the components (i.e. Young's moduli of high-CTE material over low-CTE material) play a role in the effective CTE but to a different extent. The effect of the stiffness mismatch of materials is secondary compared to that caused by a change in the skew angle, i.e. the interplay of structures. The former has a non-negligible influence only at low values of the skew angle (e.g. below $\theta=50^{\circ}$ for TL-2 concept, and below $\theta=35^{\circ}$ for TN concept). If the low-CTE material has a larger Young's modulus (i.e. $E_{s 1}<E_{s 2}$ ), an increase in $E_{s 1} / E_{s 2}$, i.e. the Young's modulus ratio of the components, reduces the effective CTE to a lower value; this phenomenon is caused by the high-CTE elements inducing a larger compensation of thermal expansion with increased stiffness. In contrast, if the high-CTE material has a larger Young's modulus (i.e. $E_{s 1}>E_{s 2}$ ), the impact of the stiffness mismatch of materials on the effective CTE is negligible. 

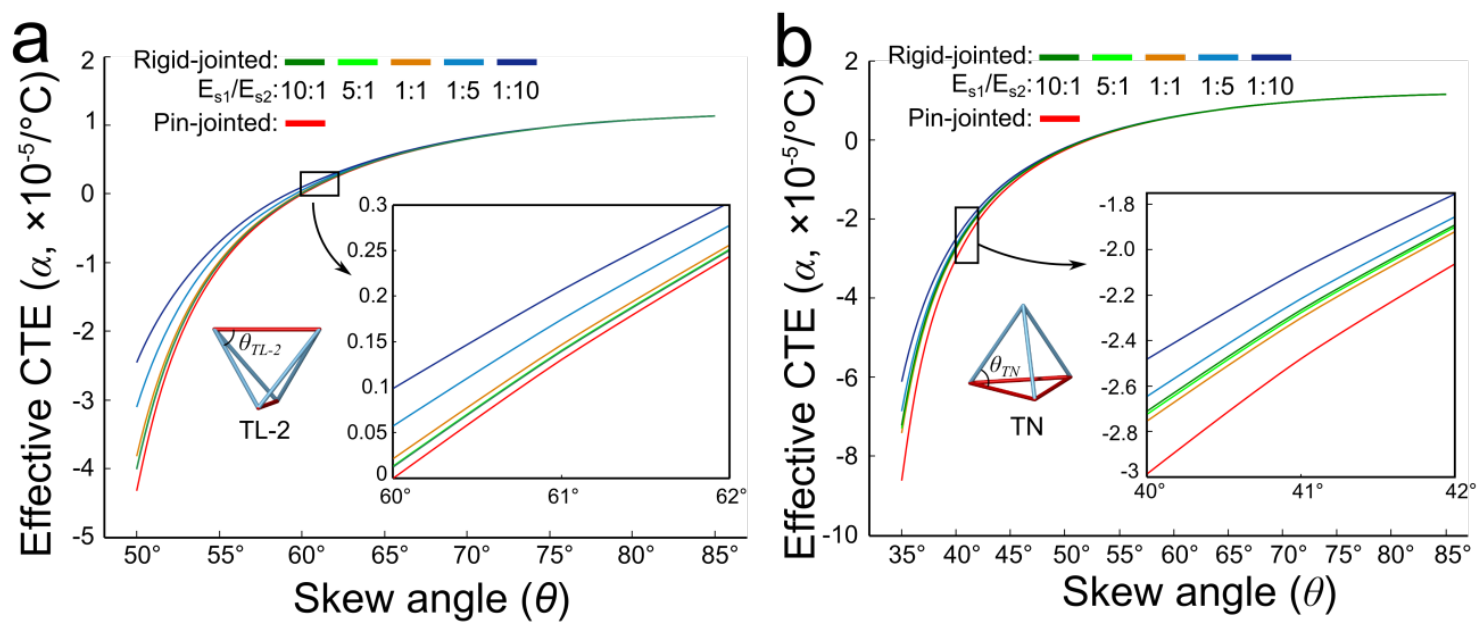

Figure A3: CTE dependence of TL-2 and TN concepts with unidirectional CTE tunability, on stiffness mismatch of materials (i.e. Young's modulus ratio of high-CTE material over low-CTE material: $E_{s 1} / E_{s 2}$ ), skew angle, and joint assumption.

\section{Appendix B: Testing setup and testing samples}

Fig. B1 shows the schematic of the experimental set-up built to test the thermal expansion of specimens. It consists of a heating chamber and a DIC system. The heating chamber with dimensions of $200(\mathrm{~L}) \times 200(\mathrm{~W}) \times 150(\mathrm{H}) \mathrm{mm}$ is made of glass to provide an unobstructed view for DIC measurements. Testing samples were set on the copper plate with thermal transfer grease (Fuchs Lubricants Co., US) between the two.
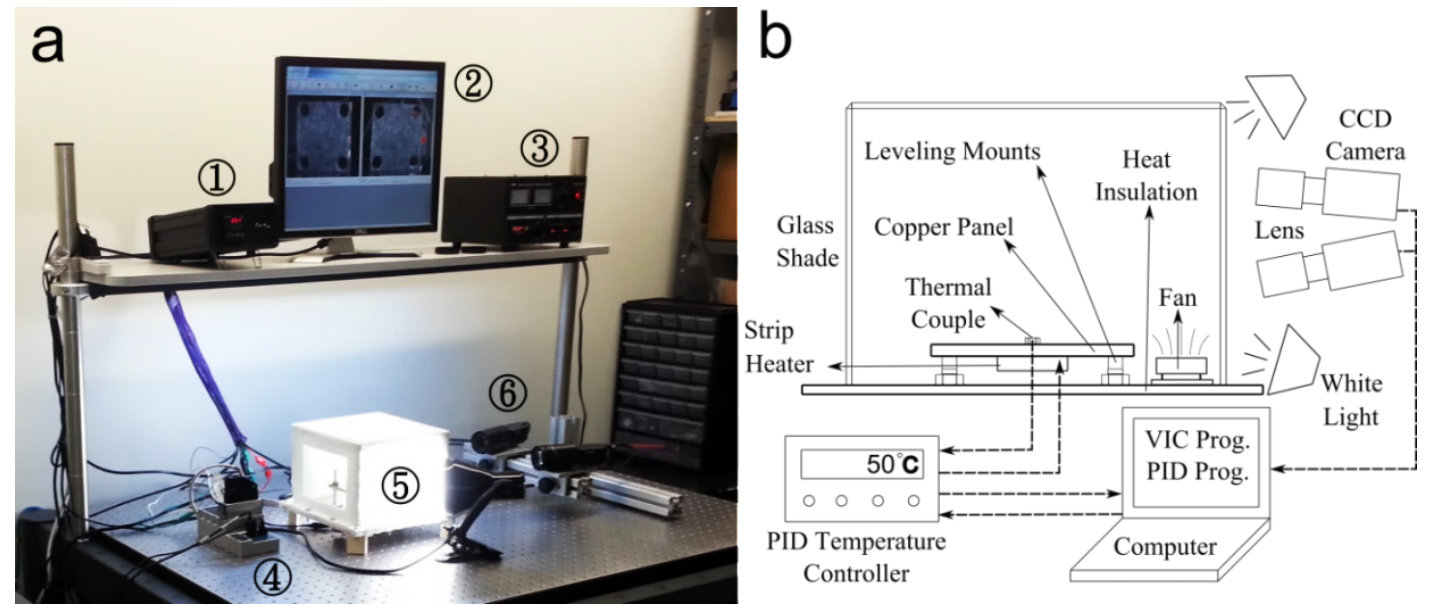

Figure B1: Experimental set-up used to measure thermal expansion: (a) 1) PID controller; 2) Computer; 3) Power source; 4) DAQ system; 5) Heating chamber; and 6) CCD cameras, and (b) schematic of the apparatus. 
Twelve physical samples (Fig. B2) were tested to validate the thermoelastic properties of the building blocks. Four sets, each of three samples, were constructed: two sets consisted of A16061 and Ti-6Al-4V (Fig. B2-I and III), and the other two were made from Al6061 and Invar (Fig. B2II and IV). Each set of material combinations included one of two configurations consisting of stationary lines or stationary nodes, at three skew angles (Fig. B2a to c). This ensures that each building block was tested using two different material combinations and three different skew angles.

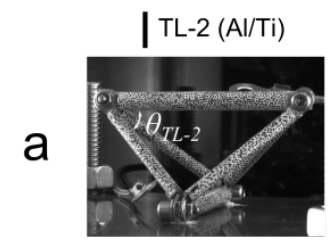

Skew angle: $53.5^{\circ}$

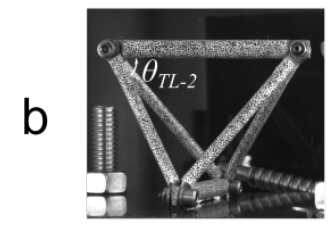

Skew angle: $61.7^{\circ}$

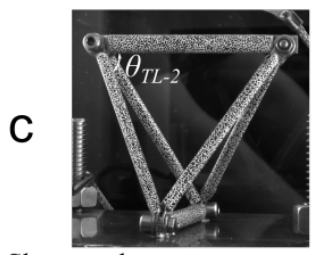

Skew angle: $63.7^{\circ}$

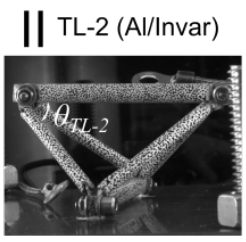

$54.1^{\circ}$

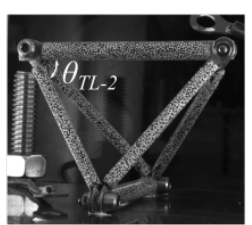

$59.6^{\circ}$

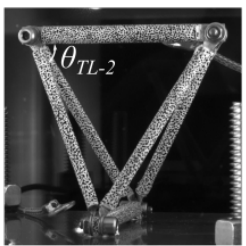

$64.9^{\circ}$

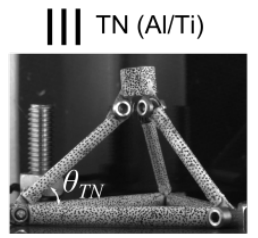

$53.7^{\circ}$

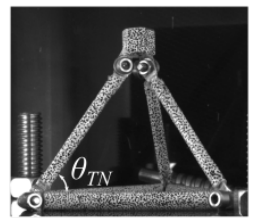

$59.7^{\circ}$

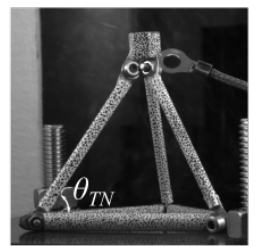

$63.3^{\circ}$

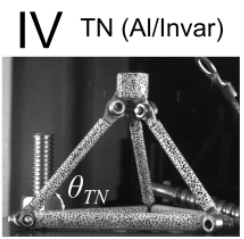

$56.2^{\circ}$

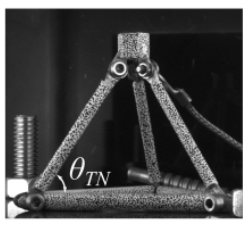

$61.0^{\circ}$

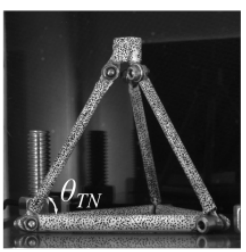

$64.0^{\circ}$

Figure B2: Thermal testing experiments for tetrahedra with stationary lines (I with $\mathrm{Al} / \mathrm{Ti}$ and II with Al/Invar) and tetrahedra with stationary nodes (III with Al/Ti and IV with Al/Invar). Samples with three skew angles of roughly $55^{\circ}(\mathrm{a}), 60^{\circ}(\mathrm{b})$, and $65^{\circ}(\mathrm{c})$ are tested.

\begin{tabular}{|l|c|c|c|c|}
\hline \multicolumn{1}{|c|}{ Sample } & $\begin{array}{c}\text { Measured CTE in } \\
\text { the vertical } \\
\text { direction } \\
\left(\times 10^{-6} /{ }^{\circ} \mathrm{C}\right)\end{array}$ & $\begin{array}{c}\text { Predicted CTE in } \\
\text { the vertical } \\
\text { direction } \\
\left(\times 10^{-6} /{ }^{\circ} \mathrm{C}\right)\end{array}$ & $\begin{array}{c}\text { Absolute } \\
\text { error } \\
\left(\times 10^{-6} /{ }^{\circ} \mathrm{C}\right)\end{array}$ & $\begin{array}{c}\text { Joint to } \\
\text { length } \\
\text { ratio } \\
(\%)\end{array}$ \\
\hline $\mathrm{TN}\left(\mathrm{Al} / \mathrm{Ti}, \theta=53.7^{\circ}\right)$ & $3.62 \pm 0.56$ & 3.97 & -0.35 & 30.8 \\
\hline $\mathrm{TN}\left(\mathrm{Al} / \mathrm{Ti}, \theta=59.7^{\circ}\right)$ & $7.65 \pm 0.45$ & 7.35 & 0.30 & 27.6 \\
\hline $\mathrm{TN}\left(\mathrm{Al} / \mathrm{Ti}, \theta=63.3^{\circ}\right)$ & $8.42 \pm 0.39$ & 8.77 & -0.35 & 26.5 \\
\hline $\mathrm{TN}\left(\mathrm{Al} / \mathrm{Invar}, \theta=56.2^{\circ}\right)$ & $-3.60 \pm 0.61$ & -3.48 & -0.12 & 30.4 \\
\hline
\end{tabular}




\begin{tabular}{|l|c|c|c|c|}
\hline TN $\left(\mathrm{Al} /\right.$ Invar, $\left.\theta=61.0^{\circ}\right)$ & $0.97 \pm 0.54$ & 0.13 & 0.84 & 29.2 \\
\hline $\mathrm{TN}\left(\mathrm{Al} /\right.$ Invar, $\left.\theta=64.0^{\circ}\right)$ & $1.69 \pm 0.25$ & 1.73 & -0.04 & 28.3 \\
\hline $\mathrm{TL}-2\left(\mathrm{Al} / \mathrm{Ti}, \theta=53.5^{\circ}\right)$ & $-11.58 \pm 0.32$ & -11.61 & 0.03 & 32.0 \\
\hline $\mathrm{TL}-2\left(\mathrm{Al} / \mathrm{Ti}, \theta=61.7^{\circ}\right)$ & $3.70 \pm 0.41$ & 4.49 & -0.79 & 30.0 \\
\hline $\mathrm{TL}-2\left(\mathrm{Al} / \mathrm{Ti}, \theta=63.7^{\circ}\right)$ & $6.47 \pm 0.68$ & 6.08 & 0.39 & 28.6 \\
\hline $\mathrm{TL}-2\left(\mathrm{Al} / \mathrm{Invar}, \theta=54.1^{\circ}\right)$ & $-26.2 \pm 0.49$ & -26.8 & 0.60 & 31.0 \\
\hline TL-2 $\left(\mathrm{Al} /\right.$ Invar, $\left.\theta=59.6^{\circ}\right)$ & $-10.40 \pm 0.64$ & -9.98 & -0.42 & 27.0 \\
\hline TL-2 $\left(\mathrm{Al} /\right.$ Invar, $\left.\theta=64.9^{\circ}\right)$ & $-2.50 \pm 0.35$ & -2.26 & -0.24 & 26.2 \\
\hline
\end{tabular}

Table B1: Predicted and experimentally measured CTE values for TN and TL-2, accompanied by their standard deviation, along with the joint length ratio, i.e. the length ratio of a copper flake to a bar, of each testing sample.

Tab. B1 tabulates the CTEs of building blocks obtained both from theory and experiments for given pair of materials. The joint-to-length ratio (averaging about 29\%), referring to the length ratio of a copper flake (made of copper with $\mathrm{CTE}$ of $16 \times 10^{-6} /{ }^{\circ} \mathrm{C}$ ) to a bar, characterizes the impact of flakes in the measured values of CTEs. Considering the joint impact, the absolute error goes no higher than $0.84 \times 10^{-6} /{ }^{\circ} \mathrm{C}$.

The nine samples of compound units are shown in Fig. B3. Randomly distributed black and white pattern is painted on the surface of ABS connection balls for DIC testing.

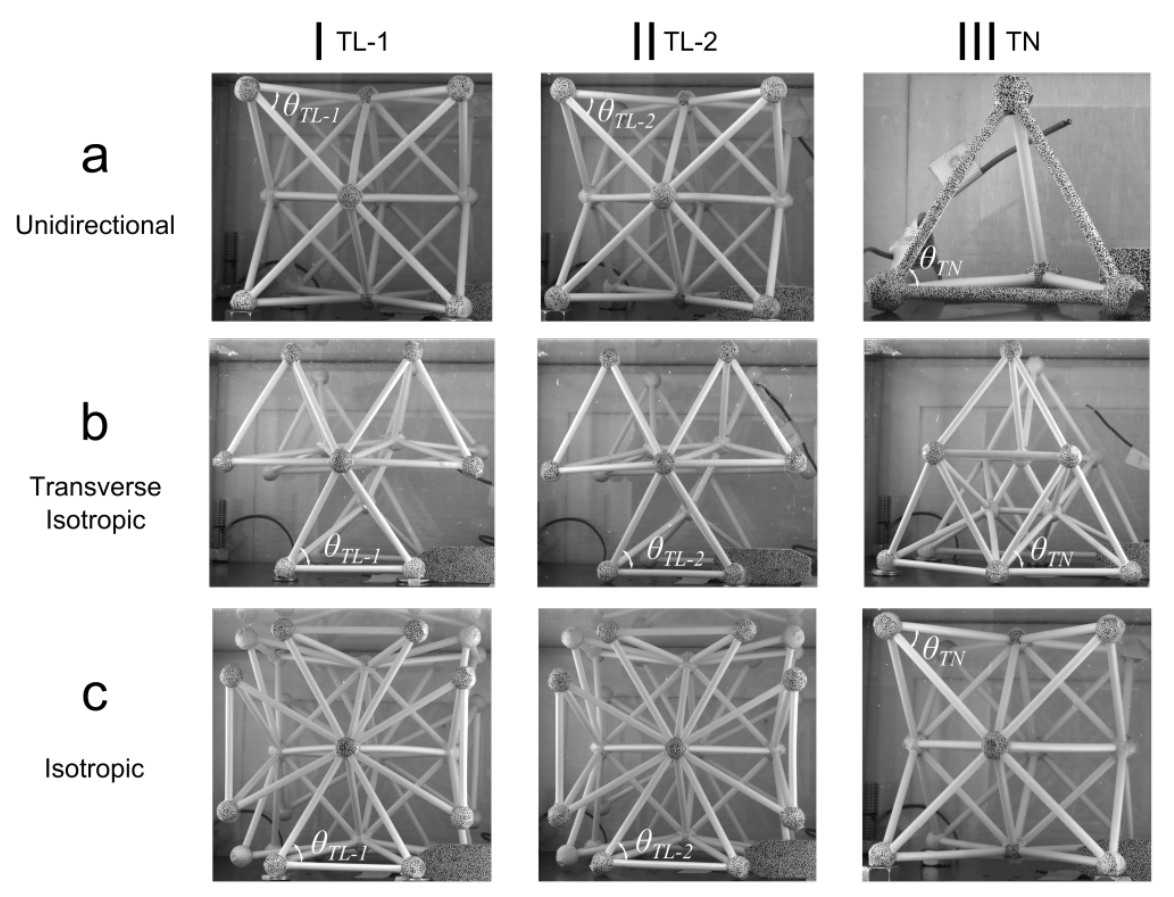


Figure B3: Thermal testing experiments for nine compound units with an identical skew angle of $60^{\circ}$.

\section{Appendix C: Calculation of elastic stiffness for truss members}

Using the standard mechanics approach here, the microscopic strain tensor, $\varepsilon_{i j}$, at any point within the representative volume element (RVE) can be linearly expressed by the macroscopic strain tensor $\overline{\varepsilon_{k l}}$ through the local structural tensor $M_{i j k l}$ :

$$
\varepsilon_{i j}=M_{i j k l} \overline{\varepsilon_{k l}} .
$$

From the constitutive relation, we have:

$$
\sigma_{i j}=C_{i j p m} \cdot \varepsilon_{p m},
$$

where $\sigma_{i j}$ is the microscopic stress tensor and $C_{i j p m}$ is the stiffness matrix of the component. The effective stress matrix can be simply defined by a volumetric average through integrating the microscopic stress over the RVE and dividing by the RVE volume:

$$
\overline{\sigma_{i j}}=\frac{1}{V_{R V E}} \int_{V_{R V E}} \sigma_{i j} d V_{R V E},
$$

where $V_{R V E}$ is the volume of the RVE. Substituting Eqs. (C.1) and (C.2) into Eq. (C.3) results in

$$
\overline{\sigma_{i j}}=\frac{1}{V_{R V E}} \int_{V_{R V E}} E_{i j p m} M_{p m k l} d V_{R V E} \overline{\varepsilon_{k l}}
$$

where $E_{i j p m}$ is the elastic properties of solid components. From Eq. (C.4) the effective stiffness matrix $\overline{C_{i j k l}}$ is defined as

$$
\overline{C_{i j k l}}=\frac{1}{V_{R V E}} \int_{V_{R V E}} E_{i j p m} M_{p m k l} d V_{R V E} .
$$

Since the RVEs of dual-material unit cells consist of discrete members, the volumetric average through integration simplifies to become a mere summation. The relation between the effective 
strain of the $k^{\text {th }}$ truss member, $\overline{\varepsilon_{p m}{ }^{(k)}}$, and the local axial strain along the bar member, $\varepsilon^{(k)}$, is given by (Mohr, 2005):

$$
\varepsilon^{(k)}=N_{i j}{ }^{(k)} \overline{\varepsilon_{i j}^{(k)}}
$$

where $N_{i j}^{(k)}$ is the linear transformation operator from the local to global coordinates of the $k^{\text {th }}$ member:

$$
N_{i j}^{(k)}=\left[\begin{array}{llllll}
l^{2} & m^{2} & n^{2} & m n & n l & l m
\end{array}\right]^{(k)}
$$

where $(l, m, n)$ are the direction cosines of the member. Furthermore, the local stress-strain relation is given by:

$$
\sigma^{(k)}=E_{s}^{(k)} \varepsilon^{(k)}
$$

where $E_{s}^{(k)}$ is the Young's modulus of the solid component of the $k^{\text {th }}$ unit cell member. The relation between the effective stress of the lattice, $\overline{\sigma_{i j}^{(k)}}$, and the local stress in the lattice member, $\sigma^{(k)}$, is given by:

$$
\overline{\sigma_{i j}^{(k)}}=\left(N_{i j}^{(k)}\right)^{\mathrm{T}} \sigma^{(k)}
$$

where $\mathrm{T}$ denotes transposition. By substituting Eqs. (C.6) and (C.8) into Eq. (C.9), we can obtain the effective stress via a summation:

$$
\overline{\sigma_{i j}}=\frac{1}{V_{R V E}} \sum_{k=1}^{k}\left(N_{i j}^{(k)}\right)^{\mathrm{T}} E_{s}^{(k)} N_{p m}^{(k)} A^{(k)} L^{(k)} \overline{\varepsilon_{p m}}
$$

where $A^{(k)}$ and $L^{(k)}$ are the cross-sectional area and length of the $k^{\text {th }}$ member, respectively. From Eq. (C.10) we can extract the macroscopic stiffness tensor component as follows: 


$$
\overline{C_{i j p m}}=\frac{1}{V_{R V E}} \sum_{k=1}^{k}\left(N_{i j}^{(k)}\right)^{\mathrm{T}} E_{s}^{(k)} N_{p m}^{(k)} A^{(k)} L^{(k)}
$$

For ease of representation of the constitutive equations, we introduce vector notation for the tensors. We write the effective stiffness tensor as:

$$
\boldsymbol{C}=\boldsymbol{N}^{\mathrm{T}} \boldsymbol{R} \boldsymbol{N},
$$

where $\boldsymbol{N}$ is the transformation operator for a lattice with $M$ sets of struts, i.e. sharing the same directional orientation, size/volume and solid component:

$$
\boldsymbol{N}=\left[\begin{array}{cccccc}
l_{1}^{2} & m_{1}^{3} & n_{1}^{2} & m_{1} n_{1} & n_{1} l_{1} & l_{1} m_{1} \\
\cdots & \cdots & \cdots & \cdots & \cdots & \cdots \\
l_{M}^{2} & m_{M}^{3} & n_{M}^{3} & m_{M} n_{M} & n_{M} l_{M} & l_{M} m_{M}
\end{array}\right],
$$

where $\left(l_{i}, m_{i}, n_{i}\right)$ are the directional cosines of the $i^{\text {th }}$ set of struts with axes $x_{1}, x_{2}$, and $x_{3}$, respectively. $\boldsymbol{R}$ is given by:

$$
\boldsymbol{R}=\left[\begin{array}{lllll}
r_{1} & & & & \\
& \ddots & & 0 & \\
& & r_{i} & & \\
& 0 & & \ddots & \\
& & & & r_{M}
\end{array}\right],
$$

with

$$
r_{i}=\frac{E_{s i} A_{i} L_{i}}{V_{R V E}}
$$

Here we examine the three unit cells with unidirectional CTE tunability (Fig. 6a to c) for demonstration purposes. The coordinate systems defined for these concepts are depicted in Fig. C1. As shown in Fig. C1, struts sharing cross sectional area and component, parallel and equally distant from each other can be considered as one set of struts. Since TL-1 and TL-2 concepts have identical strut location, they both use the coordinate system shown in Fig. C1a. 

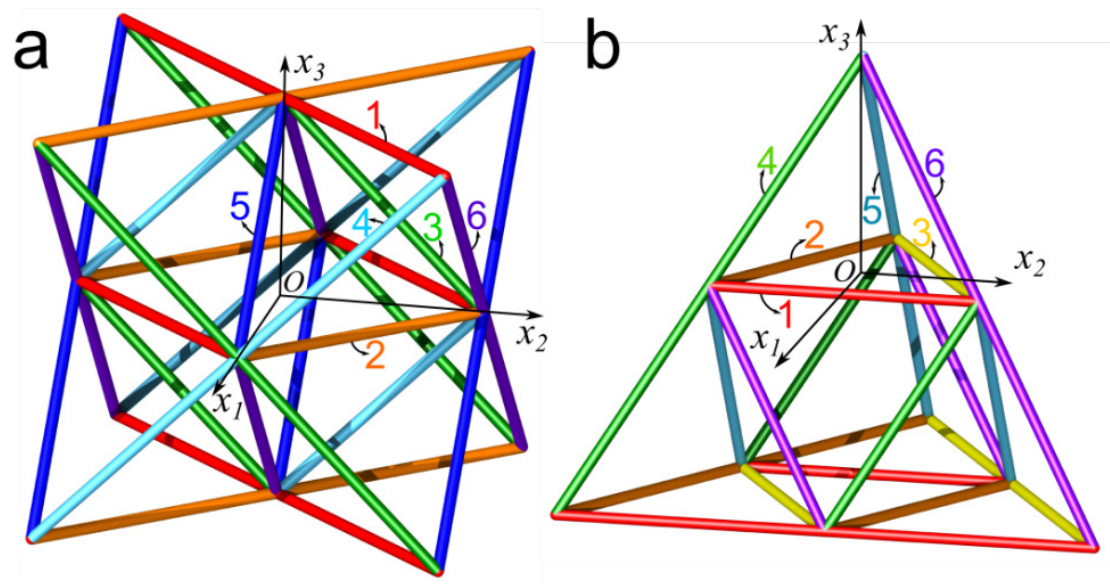

Figure 17: The coordinate system defined for (a) TL-1 and TL-2 concepts, and (b) TN concept. Different sets of trusses are marked in different colors and numbered accordingly.

For the topologies shown in Fig. 6a, b, and c, we summarized all the directional cosines, element length, and the effective Young's modulus of each set of struts, $r_{i}$, in Tab. C1 (for TL-1 and TL-2 concept) and Tab. C2 (for TN concept).

\begin{tabular}{|c|c|c|c|c|c|c|}
\hline Truss Set No. & 1 & 2 & 3 & 4 & 5 & 6 \\
\hline$l$ & $\sqrt{2} / 2$ & $\sqrt{2} / 2$ & 0 & 0 & $-\sqrt{2} \cos \theta$ & $\sqrt{2} \cos \theta$ \\
\hline$m$ & $\sqrt{2} / 2$ & $-\sqrt{2} / 2$ & $-\sqrt{2} \cos \theta$ & $\sqrt{2} \cos \theta$ & 0 & 0 \\
\hline$n$ & 0 & 0 & $\xi$ & $\xi$ & $\xi$ & $\xi$ \\
\hline Length & $l_{1}$ & $l_{1}$ & $\frac{l_{1}}{2 \cos \theta}$ & $\frac{l_{1}}{2 \cos \theta}$ & $\frac{l_{1}}{2 \cos \theta}$ & $\frac{l_{1}}{2 \cos \theta}$ \\
\hline$r$ for TL-1 & $\frac{2 E_{1} \pi a^{2} \cos \theta}{l_{1}^{2} \xi}$ & $\frac{E_{2} \pi a^{2}}{l_{1}^{2} \xi}$ & \multicolumn{5}{|c|}{$\frac{E_{2} \pi a^{2}}{l_{1}^{2} \xi}$} \\
\hline$r$ for TL-2 & \multicolumn{2}{|c|}{$\frac{2 E_{1} \pi a^{2} \cos \theta}{l_{1}^{2} \xi}$} & \multicolumn{5}{|c|}{$\frac{E_{2} \pi a^{2}}{l_{1}^{2} \xi}$} \\
\hline
\end{tabular}

Table C1: Unit vector components, lengths, and effective Young's moduli of TL-1 and TL-2 concept truss members

\begin{tabular}{|c|c|c|c|c|c|c|}
\hline Truss Set No. & 1 & 2 & 3 & 4 & 5 & 6 \\
\hline$l$ & 0 & $\frac{\sqrt{3}}{2}$ & $\frac{\sqrt{3}}{2}$ & $-\frac{\sqrt{3}}{3} \cos \theta$ & $\frac{2 \sqrt{3}}{3} \cos \theta$ & $-\frac{\sqrt{3}}{3} \cos \theta$ \\
\hline$m$ & 1 & $-\frac{1}{2}$ & $\frac{1}{2}$ & $\cos \theta$ & 0 & $-\cos \theta$ \\
\hline$n$ & 0 & 0 & 0 & $\sqrt{3} \zeta / 3$ & $\sqrt{3} \zeta / 3$ & $\sqrt{3} \zeta / 3$ \\
\hline
\end{tabular}




\begin{tabular}{|c|c|c|c|c|c|c|}
\hline Length & $l_{1}$ & $l_{1}$ & $l_{1}$ & $\frac{l_{1}}{2 \cos \theta}$ & $\frac{l_{1}}{2 \cos \theta}$ & $\frac{l_{1}}{2 \cos \theta}$ \\
\hline$r$ & \multicolumn{3}{|c|}{$\frac{4 E_{1} \pi a^{2} \cos \theta}{l_{1}^{2} \zeta}$} & \multicolumn{3}{|c|}{$\frac{2 E_{2} \pi a^{2}}{l_{1}^{2} \zeta}$} \\
\hline
\end{tabular}

Table C2: Unit vector components, lengths, and effective Young's moduli of TN concept truss members

By substituting the values of directional cosines into Eq. (C.12), we obtain the macroscopic stiffness tensor for the TL-1, TL-2, and TN concepts with unidirectional CTEs as shown in Eqs. (26) to (28).

\section{Appendix D: Yield and buckling strength}

Tetrahedral building blocks were chosen in this study because of their high structural efficiency, which includes strength/mass, in addition to stiffness/mass. For this reason, here we also examine the overall strength performance, and we do so by adopting notation and terminology used in Appendix C. In this section, the yield and buckling strength of the three concepts with unidirectional CTE tunability are derived as examples for other concepts. From Eq. (C.9), we can obtain:

$$
\overline{\sigma_{i j}^{(k)}} A^{(k)}=\left(N_{i j}^{(k)}\right)^{\mathrm{T}} \sigma^{(k)} A^{(k)}=\left(N_{i j}^{(k)}\right)^{\mathrm{T}} f^{(k)}
$$

where $f^{(k)}$ is the axial load of the $k^{\text {th }}$ bar. Considering the same simplification steps discussed earlier, the stretching forces vector, $f$, of struts can be defined as,

$$
\boldsymbol{f}=\left\{\begin{array}{lllll}
f_{1} & \cdots & f_{i} & \cdots & f_{N}
\end{array}\right\}^{\mathrm{T}}
$$

where $f_{i}$ is the axial load of the $i^{\text {th }}$ set of struts. Thus, we have

$$
S \bar{\sigma}=N^{\mathrm{T}} f
$$

with 


$$
S=\left[\begin{array}{cccccc}
S_{1} & & & & \\
& \ddots & & 0 & \\
& & S_{i} & & \\
& 0 & & \ddots & \\
& & & & S_{N}
\end{array}\right]
$$

$S_{i}$ is the area occupied by one strut in the $i^{\text {th }}$ set. From Eq. (C.12), we have

$$
\boldsymbol{I}=\boldsymbol{N}^{\mathrm{T}} \boldsymbol{R} \boldsymbol{N} \boldsymbol{C}^{-1}
$$

where $I$ is a unit matrix. Thus, we have

$$
N^{\mathrm{T}} \boldsymbol{f}=S \bar{\sigma}=N^{\mathrm{T}} R N C^{-1} S \bar{\sigma}
$$

So the stretching forces vector, $f$, can be derived as

$$
f=R N C^{-1} S \bar{\sigma}
$$

Taking the yield strength of a strut as $\sigma_{Y}$, and the Euler buckling strength as $\sigma_{E}$, the ultimate force a strut can carry is (Fan et al., 2008)

$$
f_{i j}= \begin{cases}\sigma_{Y} \pi a^{2}, & \text { yield by tension } \\ -\sigma_{Y} \pi a^{2}, & \text { yield by compression } \\ -\sigma_{E} \pi a^{2}, & \text { buckling by compression }\end{cases}
$$

The force carried by each strut can be computed according to Eq. (D. 7). If any component of vector $f$ reaches $f_{i j}$ defined in Eq. (D. 8), the corresponding global stress would be equal to the strength of the lattice material. The Euler buckling load of the struts is given by $P_{E}=n^{2} \pi^{3} a^{4} E_{s} /\left(4 l^{2}\right)$ while the plastic yielding load is $P_{Y}=\pi a^{2} \sigma_{Y}$, with $n$ determined by supporting conditions at strut ends. Thus, the critical aspect ratio is given by

$$
l_{c r}=\frac{n \pi a}{2} \sqrt{\frac{E_{s}}{\sigma_{Y}}}
$$

Under compression, if the length of the strut is larger than the critical length, then the strut will 
buckle and the buckling strength is given by

$$
\sigma_{E}=\frac{n^{2} \pi^{2} E_{s} a^{2}}{4 l^{2}}
$$

The initial yield surfaces under general macroscopic loadings are composed of the envelope of, at most, twelve super-planes in the stress space, and represented as follows for the TN concept:

$$
\begin{aligned}
& -\frac{(2 \cos \theta+1)}{2 \cos \theta} \sigma_{1}+\frac{3(2 \cos \theta+1)}{2 \cos \theta} \sigma_{2}+\frac{2 \cos \theta(2 \cos \theta+1)}{4 \cos ^{2} \theta-3} \sigma_{3}+\frac{2(2 \cos \theta+1)}{\sqrt{3-4 \cos ^{2} \theta}} \sigma_{12} \\
& \frac{(2 \cos \theta+1)}{\cos \theta} \sigma_{1}-\frac{2 \cos \theta(2 \cos \theta+1)}{\zeta^{2}} \sigma_{3}-\frac{\sqrt{3}(2 \cos \theta+1)}{\zeta} \sigma_{23}-\frac{(2 \cos \theta+1)}{\zeta} \sigma_{13}-\frac{\sqrt{3}(2 \cos \theta+1)}{\cos \theta} \sigma_{12} \\
& \left\{\frac{(2 \cos \theta+1)}{\cos \theta} \sigma_{1}-\frac{2 \cos \theta(2 \cos \theta+1)}{\zeta^{2}} \sigma_{3}+\frac{\sqrt{3}(2 \cos \theta+1)}{\zeta} \sigma_{23}-\frac{(2 \cos \theta+1)}{\zeta} \sigma_{13}+\frac{\sqrt{3}(2 \cos \theta+1)}{\cos \theta} \sigma_{12}\right. \\
& \frac{3(2 \cos \theta+1)}{\zeta^{2}} \sigma_{3}+\frac{3 \sqrt{3}(2 \cos \theta+1)}{2 \cos \theta \zeta} \sigma_{23}-\frac{(2 \cos \theta+1)}{4 \cos ^{2} \theta \zeta} \sigma_{13} \\
& \frac{3(2 \cos \theta+1)}{\zeta^{2}} \sigma_{3}+\frac{(2 \cos \theta+1)}{2 \cos ^{2} \theta \zeta} \sigma_{13} \\
& \frac{3(2 \cos \theta+1)}{\zeta^{2}} \sigma_{3}-\frac{3 \sqrt{3}(2 \cos \theta+1)}{2 \cos \theta \zeta} \sigma_{23}-\frac{(2 \cos \theta+1)}{4 \cos ^{2} \theta \zeta} \sigma_{13} \\
& = \begin{cases}\rho^{*} \sigma_{r}, & \text { yield by tension } \\
-\rho^{*} \sigma_{r}, & \text { yield by compression } \\
-\rho^{*} \sigma_{E}, & \text { buckling by compression }\end{cases}
\end{aligned}
$$

The yield criterion in the stress space for TL concepts is:

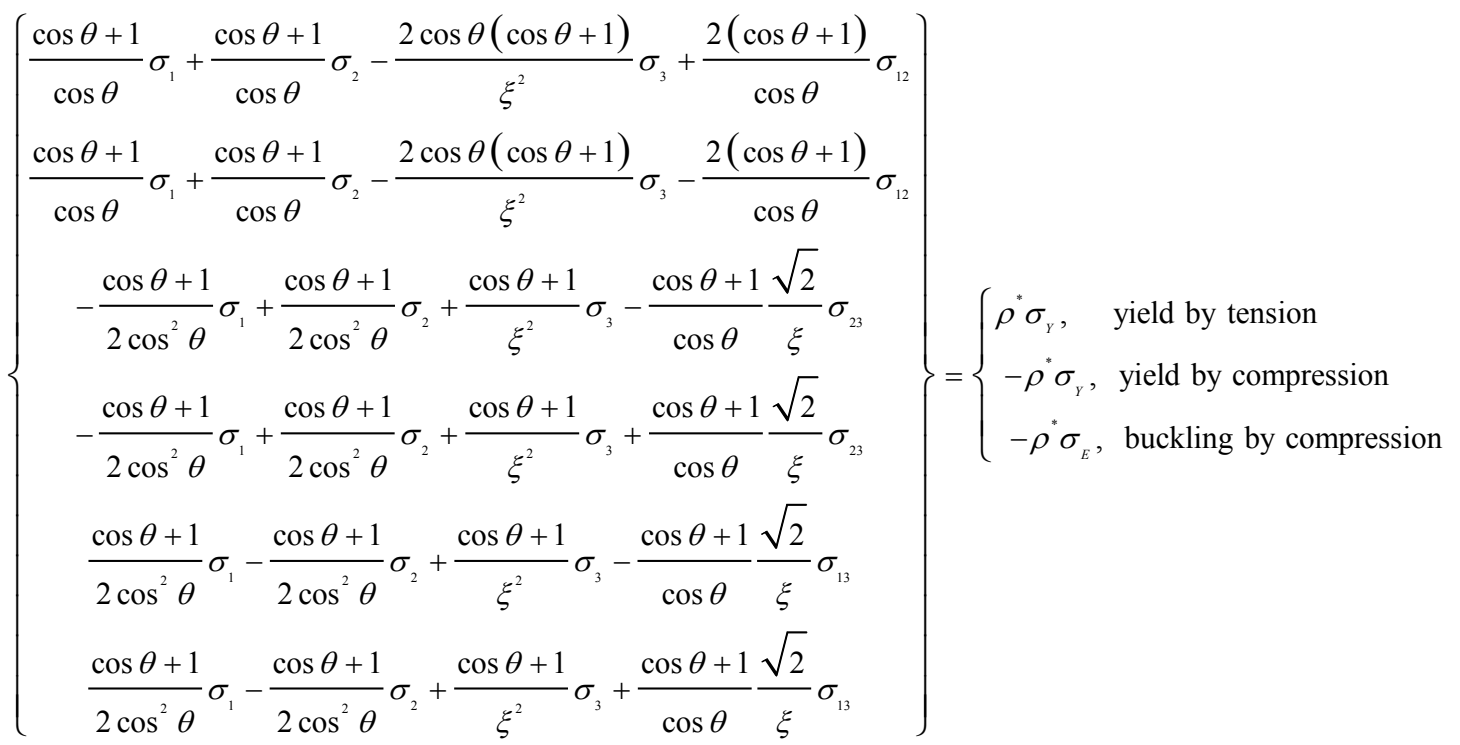


According to Eqs. (D.11) and (D.12), the yield surface of the lattice in the macroscopic $\left(\sigma_{1}, \sigma_{2}, \sigma_{3}\right)$ and $\left(\sigma_{12}, \sigma_{23}, \sigma_{13}\right)$ space is sketched in Fig. D1.
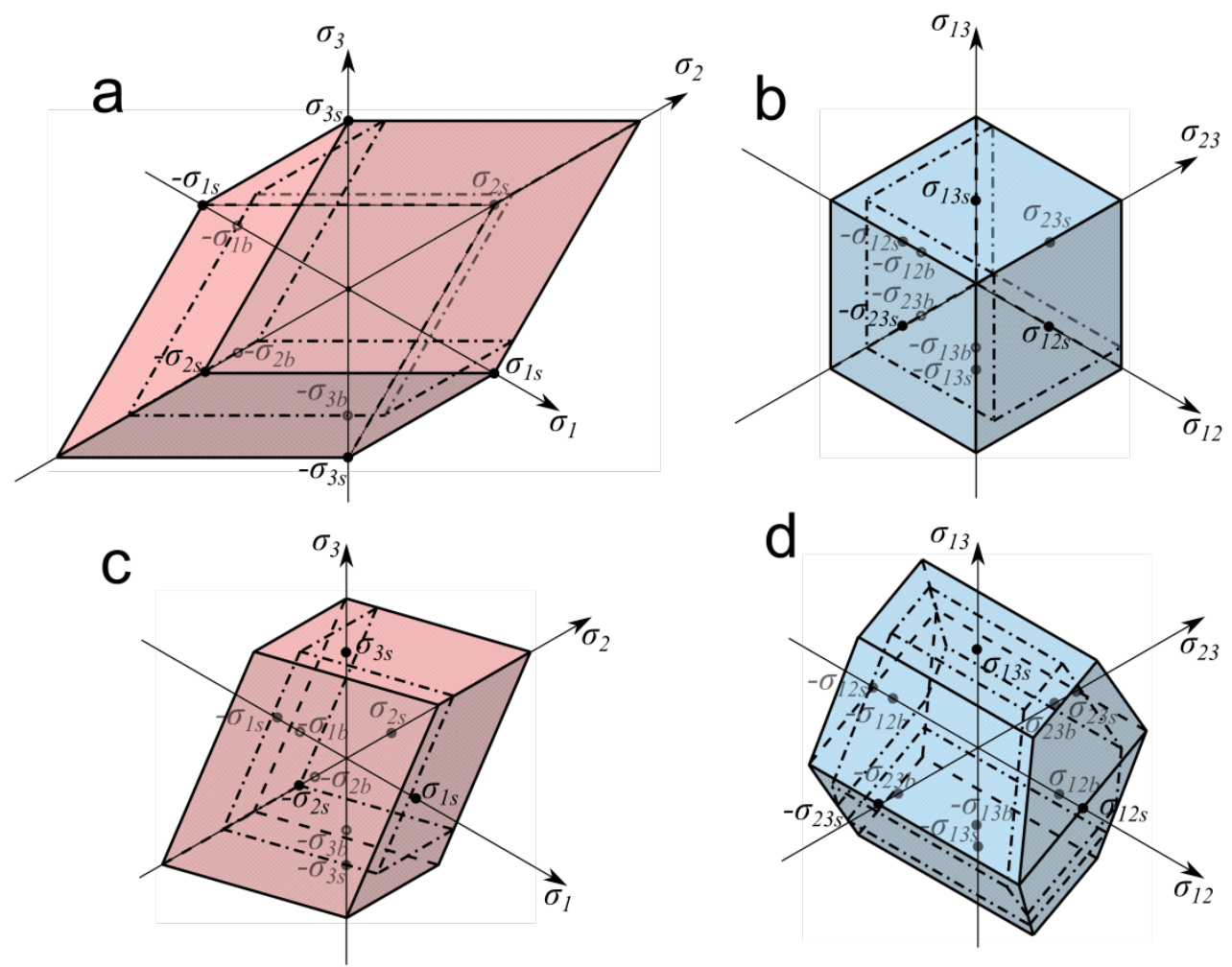

Figure 18: Yield (solid and dash line) and buckling (solid and dot dash line) surface in macroscopic $\left(\sigma_{1}, \sigma_{2}, \sigma_{3}\right)$ space: (a) for TN and (c) for TLs; and in $\left(\sigma_{12}, \sigma_{23}, \sigma_{13}\right)$ space: (b) for TN and (d) for TLs. 